\title{
Structures and Bioactivities of Steroidal Saponins Isolated from the Genera Dracaena and Sansevieria
}

\author{
Zaw Min Thu ${ }^{1, * \mathbb{D}}$, Sann Myint Oo ${ }^{1}$, Thinn Myat Nwe ${ }^{1}$, Hnin Thanda Aung ${ }^{2}$, Chabaco Armijos ${ }^{3, * \mathbb{D} \text {, }}$ \\ Faiq H. S. Hussain ${ }^{4}$ and Giovanni Vidari ${ }^{4, *}$
}

Citation: Thu, Z.M.; Oo, S.M.; Nwe, T.M.; Aung, H.T.; Armijos, C.; Hussain, F.H.S.; Vidari, G. Structures and Bioactivities of Steroidal Saponins Isolated from the Genera Dracaena and Sansevieria. Molecules 2021, 26, 1916

https://doi.org/10.3390/

molecules 26071916

Academic Editors: Maurizio Battino, Jesus Simal-Gandara and Esra Capanoglu

Received: 8 January 2021

Accepted: 23 March 2021

Published: 29 March 2021

Publisher's Note: MDPI stays neutral with regard to jurisdictional claims in published maps and institutional affiliations.

Copyright: (C) 2021 by the authors Licensee MDPI, Basel, Switzerland. This article is an open access article distributed under the terms and conditions of the Creative Commons Attribution (CC BY) license (https:// creativecommons.org/licenses/by/ $4.0 /)$.
1 Department of Chemistry, Kalay University, Kalay 03044, Myanmar; sannmyintoo@kalayuniversity.edu.mm (S.M.O.); thinnmyatnwe@kalayuniversity.edu.mm (T.M.N.)

2 Department of Chemistry, University of Mandalay, Mandalay 100103, Myanmar; hninthandaaung@mu.edu.mm

3 Departamento de Química y Ciencias Exactas, Universidad Técnica Particular de Loja, San Cayetano Alto s/n, Loja 1101608, Ecuador

4 Medical Analysis Department, Faculty of Science, Tishk International University, Erbil 44001, Iraq; faiq.hussain@ishikuniversity.onmicrosoft.com

* Correspondence: zawminthu@kalayuniversity.edu.mm (Z.M.T.); cparmijos@utpl.edu.ec (C.A.); vidari@unipv.it (G.V.); Tel.: +95-9264164150 (Z.M.T.); +593-7370-1444 (ext. 3039) (C.A.); +39-3343782582 (G.V.)

\begin{abstract}
The species Dracaena and Sansevieria, that are well-known for different uses in traditional medicines and as indoor ornamental plants with air purifying property, are rich sources of bioactive secondary metabolites. In fact, a wide variety of phytochemical constituents have been isolated so far from about seventeen species. This paper has reviewed the literature of about 180 steroidal saponins, isolated from Dracaena and Sansevieria species, as a basis for further studies. Saponins are among the most characteristic metabolites isolated from the two genera. They show a great variety in structural motifs and a wide range of biological activities, including anti-inflammatory, anti-microbial, antiproliferative effects and, in most case, remarkable cytotoxic properties.
\end{abstract}

Keywords: Dracaena; Sansevieria; phytochemical constituents; biological activities; steroidal saponins; cytotoxicity

\section{Introduction}

The dracaenoid genera Dracaena and Sansevieria are differentiated in the APG IV system of flowering plant classification and are placed in the subfamily, Nolinoideae of the family Asparagaceae of monocotyledons, in the order Asparagales [1]. However, recent molecular phylogenetic studies showed that Sansevieria was nested within Dracaena, rendering the latter paraphyletic, unless Dracaena was expanded to include species formerly placed in Sansevieria [2-4]. In this paper, we have maintained the historical division in the two genera because the chemical literature is mainly based on the former botanical classification.

The genus Dracaena comprises more than 110 accepted species. They are mostly succulent shrubs and trees that are mainly distributed in Africa, Australia, India, and Southeast Asia [5]. Dracaena species are widely used in various traditional medicine to treat different diseases, such as hemorrhoids and infections [6,7]. Dragon's blood is a deep red resin with great commercial value that is obtained from cut leaves, stems and roots from different plant taxa including about six Dracaena plants that grow in China, Southeast Asia, West Africa, Arabian Peninsula, Yemen, India, and Macaronesia [8,9]. The resin has remarkable anti-inflammatory and antioxidant effects and it has widely been used in herbal medicines through the world for thousands of years as an efficacious remedy for the treatment of hemorrhage, dysentery, diarrhea, stomach and external ulcers, wounds, leucorrhea, rheumatism, fractures, piles, diabetes, and even tumors [9-13]. 
The genus Sansevieria comprises about 70 herbaceous species with rhizomatous roots, with a distribution ranging from Africa through Asia to Myanmar and the Islands of the Indian Ocean $[14,15]$. Leaves and rhizomes are used in folk medicine for treating asthma, cough, sexual weakness, hypertension, diarrhea, hemorrhoids, abdominal pains, colics, eczema, piles, edema, jaundice, anuria, palpitations, viral hepatitis, malaria, snake- and insect bites, etc. [16-19]. In addition to usages as herbal remedies, several Dracaena and Sansevieria species have great horticultural importance and are commercialized for use in landscaping and as indoor ornamental plants [20]. Moreover, it has been reported that Dracaena spp. can be used as bioindicators for the control of increasing air pollution in urban cities [21], whereas the leaf fiber of many Sansevieria are used for making fine matting, rope or cordage, and articles of clothing [14].

The most characteristic secondary metabolites isolated from Dracaena and Sansevieria are steroids, flavonoids, stilbenes, and saponins; flavonoids and saponins form the largest groups. The structures and bioactivities of phenolic constituents, flavonoids and stilbenoids have been reported in recent reviews [8,22]. On the other hand, there is great interest in saponins due to their intriguing structures and the potent analgesic, anti-inflammatory, antimicrobial, antioxidant, antiproliferative, hypocholesterolemic, and cytotoxic activities [23-25]. This paper describes the about 180 different saponins isolated from 14 Dracaena and 3 Sansevieria species that have been investigated so far for these metabolites. Biological activities have been summarized at the end of the paper. Most structures have been determined in the last twenty years. The literature has been retrieved from the databases Reaxys ${ }^{\circledR}$, Scifinder ${ }^{\circledR}$, and Google Scholar until the end 2020. A partial list of saponins isolated from Dracaena and Sansevieria species has been included in an earlier review on the Agavaceae family [23].

\section{General Aspects of Steroidal Saponins Isolated from Dracaena and Sansevieria Species}

Saponins are a group of plant glycosides characterized by their high surfactant properties. Therefore, they form stable soap-like foams in aqueous solution and are highly toxic if injected into the bloodstream because of powerful hemolytic effects, due to their capacity to alter membrane permeability. Saponins are classified as steroidal or triterpenoid saponins depending on the nature of the aglycone, called sapogenin. Steroidal glycosides [24], as those found in Dracaena and Sansevieria species, are naturally occurring sugar conjugates of a steroid core. Spirostanol and furostanol saponins are largely predominant in Dracaena and Sansevieria species. Their sapogenin cores are those of $C_{27}$ sterols, biogenetically derived from cholesterol, in which oxidations of carbons C-16, C-22 and C-26 give rise to either a spiroacetal (spirostanol sapogenins) or a hemiacetal moiety (furostanol sapogenins), as shown in Figure 1. Thus, the spirostanol skeleton has a tetrahydrofuran ring (E) and a tetrahydropyran ring $(\mathrm{F})$ joined at $\mathrm{C}-22$ to give a spirane moiety, whereas the furostanol skeleton contains a tetrahydrofuran ring $(\mathrm{E})$.

Other modifications of the steroid skeleton involve oxidation at C-1 (e.g., compound 1), C-23 (e.g., 15), C-24 (e.g., 9), C-25 (e.g., 119) and, less frequently, oxidation at C-4 (e.g., 137), C-5 (e.g., 177), C-6 (e.g., 24), C-7 (e.g., 5), C-12 (e.g., 23), C-14 (e.g., 92), C-17 (e.g., 7), C-23 (e.g., 177) and C-27 (e.g., 96), introduction of a double bond between C-25 and C-27 (e.g., 1) and, more rarely, between C-20 and C-22 (e.g., 140). Furostanol acetals bearing a methoxy group at C-22 (e.g., 133) are usually considered artifacts generated from the corresponding 22-OH derivatives as a result of extraction and processing of the plant materials with $\mathrm{MeOH}$ [24]. Rare $\mathrm{C}_{27}$ cholestane derivatives (e.g., 176), as well as $\mathrm{C}_{22}$ (e.g., 165) and $C_{21}$ (e.g., 168) pregnane steroidal sapogenins, arising from the oxidative rupture of the C-22/C-23, and C-20/C-22 bonds, respectively, of a cholestane precursor have also been isolated from a few Dracaena and Sansevieria species. 

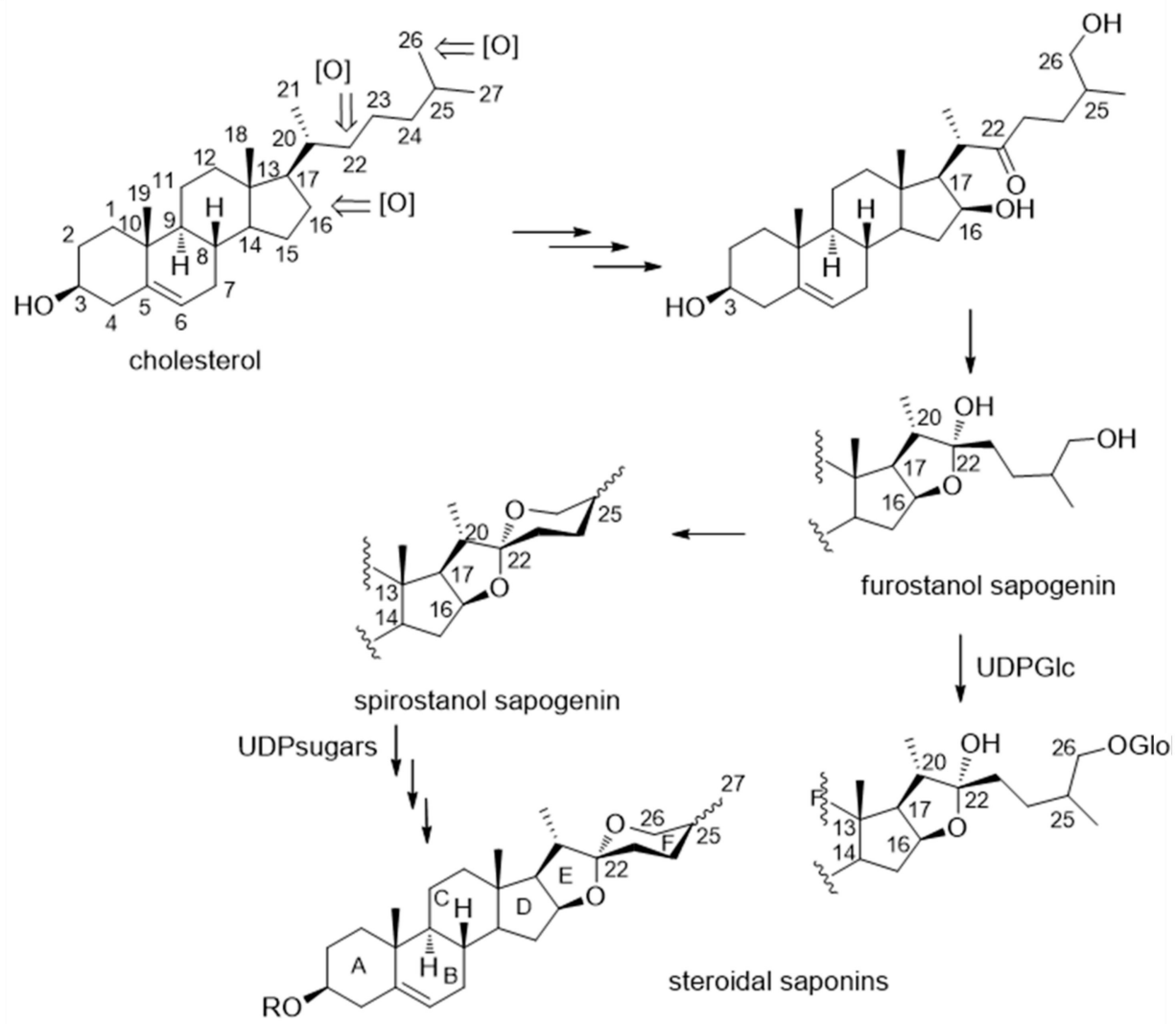

Figure 1. Main steps in the biosynthetic transformation of cholesterol to steroidal saponins.

In relation to the stereochemistry of the sapogenin nucleus, the C-3, C-8, C-9, C-10, C-13, C-14, C-17, and C-20 centers retain the same stereochemistry as the parent cholesterol almost invariably. Whereas, the configurations of oxygenated substituents at C-1, $\mathrm{C}-16$, and $\mathrm{C}-22$ are $R, S$, and $R$, respectively, with the exception of saponin 90 that is $22 S$. $\mathrm{C}-23$ stereocenters bearing a $\mathrm{OH}$ have the $\mathrm{R}$ configuration, while the $\mathrm{C}-24$ and $\mathrm{C}-25$ stereocenters may have either the $R$ or $S$ configuration. A mixture of stereoisomers can also occur in the same plant.

\subsection{Glycosidic Moieties of Dracaena and Sansevieria Saponins}

The glycone parts of Dracaena and Sansevieria saponins can be mono-, di-, tri- and tetraglycosides, depending on the number of sugar units that may include $\beta$-D-glucopyranosyl $(\beta$ D-Glc $p$ ), $\alpha$-L-rhamnopyranosyl ( $\alpha$-L-Rhap), $\alpha$-L-arabinopyranosyl ( $\alpha$-L-Ara $p$ ), $\beta$-D-xylopyranosyl $(\beta$-D-Xyl $p)$, and $\beta$-D-fucopyranosyl ( $\beta$-D-Fuc $p$ ) moieties (Table 1$)$.

Table 1. Glycosylation patterns of Dracaena and Sansevieria saponins. ${ }^{\mathrm{a}, \mathrm{b}}$

\begin{tabular}{|c|c|c|c|c|c|c|c|c|c|c|c|c|}
\hline & $1-\mathrm{OH}$ & 3- & 6- & 12- & 15- & 16- & 24- & 26- & $2^{\prime}-$ & $3^{\prime}-$ & $4^{\prime}-$ & $4^{\prime \prime}-$ \\
\hline $\begin{array}{l}\text { Spirostanol } \\
\text { monoglyco- } \\
\text { sides }\end{array}$ & $\begin{array}{c}\text { Arap } \\
3 \\
\text { Xylp } 1\end{array}$ & Glcp 3 & - & - & - & - & - & - & - & - & - & - \\
\hline $\begin{array}{l}\text { Spirostanol } \\
\text { diglyco- } \\
\text { sides }\end{array}$ & $\begin{array}{c}\text { Arap } 19 \\
\text { Glcp } p \\
3 \\
\text { Fucp } 3 \\
\text { Xyl } p \\
1\end{array}$ & $\begin{array}{l}\text { Galp1 } \\
\text { Glcp } \\
15\end{array}$ & Glcp 1 & - & - & - & Glcp 3 & - & $\begin{array}{c}\text { Glcp } \\
1 \\
\text { Rhap } \\
28\end{array}$ & $\begin{array}{c}\text { Rhap } 1 \\
\text { Xylp } \\
1\end{array}$ & $\begin{array}{c}\text { Galp } \\
1 \\
\text { Rhap } 6\end{array}$ & - \\
\hline
\end{tabular}


Table 1. Cont.

\begin{tabular}{|c|c|c|c|c|c|c|c|c|c|c|c|c|}
\hline & $1-\mathrm{OH}$ & $3-$ & 6- & $12-$ & $15-$ & $16-$ & 24- & 26- & $2^{\prime}-$ & $3^{\prime}-$ & $4^{\prime}-$ & $4^{\prime \prime}-$ \\
\hline $\begin{array}{l}\text { Spirostanol } \\
\text { triglyco- } \\
\text { sides }\end{array}$ & $\begin{array}{c}\text { Arap } 23 \\
\text { Fucp } 2 \\
\text { Glc } p \\
3 \\
\text { Xyl } p 1\end{array}$ & $\begin{array}{l}\text { Glcp } \\
32\end{array}$ & - & - & - & - & $\begin{array}{c}\text { Arap } 1 \\
\text { Fucp } 5 \\
\text { Glcp } \\
1\end{array}$ & - & $\begin{array}{l}\text { Rhap } \\
56 \\
\text { Glcp } \\
1\end{array}$ & $\begin{array}{c}\text { Galp } 1 \\
\text { Glcp } 8 \\
\text { Rhap } 9 \\
\text { Xylp } \\
21\end{array}$ & $\begin{array}{c}\text { Apif } \\
1 \\
\text { Ara } p 1 \\
\text { Rha } p 7 \\
\text { Xylp } 1\end{array}$ & Galp 3 \\
\hline $\begin{array}{l}\text { Spirostanol } \\
\text { tetraglyco- } \\
\text { sides }\end{array}$ & $\begin{array}{l}\text { Arap } 13 \\
\text { Fucp } 1\end{array}$ & - & - & - & - & - & $\begin{array}{c}\text { Arap } 1 \\
\text { Fucp } \\
10 \\
\text { Glcp } \\
2 \\
\text { Rhap } 1\end{array}$ & - & $\begin{array}{c}\text { Rhap } \\
13 \\
\text { Xylp } 1\end{array}$ & $\begin{array}{c}\text { Rhap } 1 \\
\text { Xylp } \\
13\end{array}$ & - & - \\
\hline $\begin{array}{c}\text { Furostanol } \\
\text { diglyco- } \\
\text { sides }\end{array}$ & $\begin{array}{c}\text { Fucp } 2 \\
\text { Glcp } \\
2\end{array}$ & - & - & - & - & - & - & $\begin{array}{l}\text { Glcp } \\
5\end{array}$ & $\begin{array}{c}\text { Glcp } \\
\quad 1\end{array}$ & - & - & - \\
\hline $\begin{array}{l}\text { Furostanol } \\
\text { triglyco- } \\
\text { sides }\end{array}$ & Arap 5 & $\begin{array}{l}\text { Arap } 2 \\
\text { Fucp } 4 \\
\text { Glc } p \\
7\end{array}$ & - & - & - & - & - & $\begin{array}{c}\text { Glcp } \\
15 \\
\text { Rhap } 1\end{array}$ & $\begin{array}{l}\text { Rhap } \\
15\end{array}$ & $\begin{array}{c}\text { Glcp } \\
1\end{array}$ & Rhap 4 & - \\
\hline $\begin{array}{l}\text { Furostanol } \\
\text { tetraglyco- } \\
\text { sides }\end{array}$ & $\begin{array}{l}\text { Arap } 6 \\
\text { Fucp } 1 \\
\text { Glcp } 4 \\
\text { Xylp } 1\end{array}$ & $\begin{array}{l}\text { Glcp } \\
18\end{array}$ & - & $\begin{array}{c}\text { Rhap } \\
1\end{array}$ & Rhap 1 & - & - & $\begin{array}{l}\text { Glcp } \\
30\end{array}$ & $\begin{array}{c}\text { Rhap } \\
29\end{array}$ & $\begin{array}{l}\text { Glcp } 6 \\
\text { Xylp } \\
13\end{array}$ & $\begin{array}{c}\text { Rhap } \\
10\end{array}$ & - \\
\hline $\begin{array}{l}\text { Cholestane } \\
\text { derivatives }\end{array}$ & $\begin{array}{c}\text { Arap } 1 \\
\text { Rhap } \\
1\end{array}$ & $\begin{array}{l}\text { Glcp } \\
\quad 4\end{array}$ & & & & $\begin{array}{c}\text { Glcp } \\
1\end{array}$ & & $\begin{array}{c}\text { Glcp } \\
1\end{array}$ & Rhap 3 & Rhap 2 & - & - \\
\hline $\begin{array}{c}\text { Pregnanes } \\
\text { and } \\
\text { lactones } \\
\text { 165-167 }\end{array}$ & $\begin{array}{c}\text { Arap } 3 \\
\text { Glcp } \\
2\end{array}$ & $\begin{array}{c}\text { Glcp } \\
6\end{array}$ & & & & & & $\begin{array}{c}\text { Glcp } \\
\quad 1\end{array}$ & $\begin{array}{c}\text { Rhap } \\
11\end{array}$ & $\begin{array}{l}\text { Glcp } 2 \\
\text { Rhap } 2 \\
\text { Xylp } 3\end{array}$ & Rhap 2 & \\
\hline
\end{tabular}

${ }^{a}$ The number near each sugar code indicates the number of saponins containing that sugar bonded to the indicated $\mathrm{OH} .{ }^{b}$ For the calculation, each stereoisomer in a mixture has been considered separately.

One saponin (48) contains a $\beta$-D-apiofuranosyl ( $\beta$-D-Apif) unit and four compounds (23, 73-75) contain $\beta$-D-galactopyranosyl ( $\beta$-D-Gal $p$ ) residues. Noteworthy, no furostanol monoglycoside has been isolated so far from Dracaena and Sansevieria species while also spirostanol monoglycosides are rare. Spirostanol di- and triglycosides, as well as furostanol tetraglycosides, are the most abundant saponins in Dracaena and Sansevieria. The sugar moieties can be present at one (monodesmosidic saponins) or two different positions (bisdesmosidic saponins) of the sapogenin core. One rare tetradesmodic saponin (152) is also known. Tri- and tetraglycoside units are branched with the exception of the linear triglycosides 73-75.

Sugar units are attached through an acetal linkage to a hydroxyl group on the steroid core. The most common glycosylation sites are $1-\mathrm{OH}, 3-\mathrm{OH}$, and $24-\mathrm{OH}$ in spirostanes, and $1-\mathrm{OH}, 3-\mathrm{OH}$, and $26-\mathrm{OH}$ in furostanes (Table 1). The hydroxyl groups, 24-, 25- and 26-OH are usually monoglycosylated, whereas a branched oligosaccharide moiety is commonly attached to the other sites. An $\alpha$-L-arabinopyranosyl residue is commonly attached to $1-\mathrm{OH}$, whereas $3-\mathrm{OH}$ and $26-\mathrm{OH}$ are mainly $\beta$-D-glucosylated; a $\beta$-D-fucopyranosyl moiety is the typical substituent of $24-\mathrm{OH}$ (Table 1). In branched oligosaccharides, an $\alpha$-L-rhamnopyranosyl group is almost invariably attached to $2^{\prime}-\mathrm{OH}$, whereas a $\beta$-D-xylopyranosyl unit, an $\alpha$-L-rhamnopyranosyl group or, less frequently, a $\beta$ D-glucopyranosyl residue is bonded to $3^{\prime}-\mathrm{OH}$. An $\alpha$-L-rhamnopyranoside moiety is also the common substituent at $4^{\prime}-\mathrm{OH}$. Interestingly, $\beta$-D-fucopyranose and $\alpha$-L-arabinopyranose, except for saponin 84, have never been found as inner components of branched oligosac- 
charides in Dracaena and Sansevieria saponins. $\beta$-D-Galactopyranose has been identified as the terminal sugar of linear trisaccharide moieties (e.g., 74).

Acetyl (e.g., 15) and, more rarely, sulfatyl (e.g., 6) groups may be present as part of the sugar moieties.

\subsection{Isolation and Structure Determination}

There is not a general specific procedure for the isolation of steroidal saponins from Dracaena and Sansevieria; instead, methods are analogous to those used for other natural saponins [24], for example from Agave species [25]. Based on our personal experience and key references in the literature [26-33], some general guidelines can, however, be suggested. Soon after collection, vegetal material is quickly air-dried in the shade to avoid enzymatic or microbial degradation and is then minced and cold- or heat-extracted by maceration or in a Soxhlet with $\mathrm{MeOH}$ or $\mathrm{EtOH}$, or with $40-70 \%$ aqueous alcohol. The vegetal material is often defatted with a hydrocarbon solvent before the extraction with alcohol. Using $\mathrm{MeOH}$, 22-OH furostanol glycosides are usually converted into their respective 22-methoxyl derivatives [31]. The presence of spirostanol and furostanol glycosides in the extract can be revealed by yellow spots on TLC plates sprayed with $1 \%$ anisaldehyde and $10 \%$ sulfuric acid in $\mathrm{MeOH}$, followed by heating at $100^{\circ} \mathrm{C}$. Moreover, furostanol glycosides stain red with the Ehrlich reagent (1\% 4-dimethylamino benzaldehyde and $10 \% \mathrm{HCl}$ in $\mathrm{MeOH}$ ), while spirostanol glycosides are colorless. To our knowledge, hyphenated techniques such as HPLC coupled with mass spectrometry (LC-MS) or with nuclear magnetic resonance spectroscopy (LC-NMR), and capillary electrophoresis coupled with mass spectroscopy (CEMS), have not been used until now for the rapid screening of the extracts/fractions for the presence and identification of saponins from Dracaena and Sansevieria.

Saponins may sometimes be precipitated from the crude extract by adding an excess of acetone, but usually the extract, after filtration, is carefully concentrated under reduced pressure in a rotary evaporator, paying attention to the inevitable formation of troublesome foams. To avoid excessive foaming and bumping, the rotation speed of the evaporation flask shall be moderate, and the use of a splash head and a vacuum regulator is highly recommended. The syrupy residue is then suspended in water and partitioned directly with $n$-BuOH or, for a preliminary defatting and fractionation, with hexane or $\mathrm{Et}_{2} \mathrm{O}$ or $\mathrm{CH}_{2} \mathrm{Cl}_{2}$, followed by EtOAc and $n-\mathrm{BuOH}$, in the order. Saponins usually migrate to the $n$ - $\mathrm{BuOH}$ soluble fraction; however, some amounts of the less polar monoglycosyl derivatives can also be extracted by EtOAc, while the most polar glycosides may remain in the aqueous layer. After concentration of these extracts under reduced pressure, the separation of a mixture of polar saponins into individual components is usually tedious and highly challenging. The traditional purification and separation procedures generally consist of fractionation over Sephadex LH-20 columns, followed by repetitive separations on silica gel columns using chloroform-methanol and/or chloroform-methanol-water in various ratios as eluents. Chromatographic separations on reverse-phase (e.g., RP-18 or Diaion ${ }^{\circledR}$ HP-20) columns using a gradient of $\mathrm{H}_{2} \mathrm{O}-\mathrm{MeOH}$ or $\mathrm{H}_{2} \mathrm{O}-\mathrm{MeCN}$ as eluent are often performed. Preparative or semipreparative MPLC or even HPLC using analytical columns, and/or preparative TLC, are usually applied in the final steps of the purification process. Droplet countercurrent chromatographic (DCCC) techniques often give excellent separations, although the development of optimal conditions may be troublesome. At the end of the purification process, an isolated crystalline compound should be recrystallized, whenever possible.

Subsequently, the structure of a homogeneous saponin is established by a combination of chemical and spectroscopic methods [24-33]. Chemical reactions are sample demanding and usually require tens of milligrams of a saponin, while spectra can be determined on a few milligrams of a substance.

For a known saponin or sapogenin, comparison of the NMR spectra, melting point, and specific rotation with the literature is generally adequate to establish the identity. However, care must be used on comparing NMR spectra which have been determined 
in different solvents, as significant differences may exist. In fact, there is no general consensus on the best solvent to use, so that pyridine- $d_{5}$, methanol- $d_{4}$ and DMSO- $d_{6}$ are all used indifferently.

The procedure for establishing the structure of a novel saponin usually involves the determination of: (i) The structures of sugar residues and the steroid aglycone; (ii) the sugar sequence; (iii) the glycosylation pattern; (iv) molecular stereochemistry.

Hydrolysis of the saponin under strong acid conditions $(1 \mathrm{~N} \mathrm{HCl}, \mathrm{MeOH}$ or dioxane$\mathrm{H}_{2} \mathrm{O}, 1: 1,0.5-1 \mathrm{mg} / \mathrm{mL}, 80-90^{\circ} \mathrm{C}$, h; or $2 \mathrm{~N}$ aqueous $\mathrm{CF}_{3} \mathrm{COOH}, 3 \mathrm{mg} / 5 \mathrm{~mL}, 95^{\circ} \mathrm{C}, 3 \mathrm{~h}$ ) allows for the identification of the aglycone and the monosaccharide constituents separately. However, the possibility that the hydrolysis products are not natural sapogenins but are artifacts, should be considered. Individual monosaccharides and their ratio in the saponin are determined by TLC (anisaldehyde/sulfuric acid, followed by heating for the spot visualization), (enantioselective) GLC (alditol acetates or trifluoroacetates/TMS derivatives), (enantioselective) HPLC with a Refractive Index detector, optical rotation, and comparison with authentic samples. Partial hydrolysis (e.g., by $0.2 \mathrm{~N} \mathrm{HCl}$, dioxane $-\mathrm{H}_{2} \mathrm{O}, 1: 1,95{ }^{\circ} \mathrm{C}$, $30 \mathrm{~min}-2 \mathrm{~h}$ ), followed by isolation and characterization of prosapogenin and oligosaccharides, is employed for the determination of terminal sugars and sugar sequences [24]. A $\beta$-glucosidase enzyme can also be employed to hydrolyze the $\beta$-glucosidic linkage(s) of a glucoside. Interestingly, cleavage of a furostanol 26-O-glucoside by a furostanol glycoside $26-O-\beta$-glucosidase affords directly the corresponding spirostane and $\beta$-D-glucose [24]. When acetyl or sulfate groups are present on the sugar moieties of a saponin, acetates are cleaved by treatment with $5 \% \mathrm{NaOMe}$ or $\mathrm{K}_{2} \mathrm{CO}_{3}$ in $\mathrm{MeOH}$ or $10 \%$ ammonia solution in $\mathrm{MeOH}$, whereas sulfate groups, identifiable by a strong $\mathrm{S}=\mathrm{O}$ band at $1230 \mathrm{~cm}^{-1}$, can be detached by refluxing with a mixture of pyridine and dioxane [24]. The sites of a sapogenin where different sugar units are attached can be revealed by permethylation of the glycoside, followed by hydrolysis and identification of the partially methylated sugars and sapogenin by GLC and NMR spectroscopy, respectively. Hydrolysis of a permethylated saponin, followed by GC-MS analysis of the methylated sugar mixture after reduction $\left(\mathrm{NaBH}_{4}\right)$ and acetylation $\left(\mathrm{Ac}_{2} \mathrm{O} / \mathrm{Py}\right)$, is used for determining interglycosidic linkages.

As regards spectrometric methods, they are powerful and indispensable tools for the structure elucidation of saponins. Different ion peaks appearing in spectra obtained by soft-ionizing methods, such as FD-MS, ESI-MS, FAB-MS, (ESI-TOF)-MS in conjunction with multi-stage tandem mass spectrometry, not only provide the correct molecular weight but also, in many instances, the sequence of sugars in the oligosaccharide moiety, through study of the fragmentation pathways. Both negative and positive ion modes have been used to obtain the molecular weight of saponins. The loss of various monosaccharide units from a saponin molecule generates fragmentation peaks with a characteristic $m / z$ value. Therefore, loss of fragments of 132, 146 and 162 mass units indicates cleavage of pentose (arabinose or xylose), deoxyhexose (rhamnose or fucose) and hexose (glucose or galactose) moieties, respectively. Of course, the information inferred from MS methods must be used in conjunction with ${ }^{1} \mathrm{H}$ and ${ }^{13} \mathrm{C}$ NMR spectroscopy (glycosidation and esterification shift rules, comparison of NMR data, utilization of the $J^{3}$ values between $\mathrm{H}-1$ and H-2 for determining the anomeric configuration) together with chemical strategies (see above) [24]. In fact, modern NMR techniques are the methods of choice for establishing the structure of the aglycone, the nature and number of the constituent sugar units including their ring sizes, anomeric configurations, interglycosidic linkages as well as the point(s) of attachment of the sugar chain to the sapogenin. Determination of all standard $1 \mathrm{D}$ and $2 \mathrm{D}{ }^{1} \mathrm{H}$ and ${ }^{13} \mathrm{C}-\mathrm{NMR}$ spectra are usually necessary to firmly establish the structure of a new saponin.

The most characteristic spectroscopic data of saponins are briefly summarized below. In addition, the consultation of the reference literature $[24,25,34,35]$ is highly recommended.

\subsection{NMR Spectra of Dracaena and Sansevieria Saponins}

In the ${ }^{1} \mathrm{H}$ NMR spectra of saponins, two singlets and two doublets occurring in the range of $\delta$ 0.5-1.7 are diagnostic for the methyl groups at C-10, C-13, C-20, and C-25 of a 
steroid nucleus. In the lower part of the same NMR region, an additional doublet can be attributed to the methyl group of a 6-deoxy-sugar residue, such as a rhamnosyl unit.

\subsubsection{Type of Parent Skeleton and Stereochemistry at C-22}

The chemical shift of $\mathrm{C}-22$ in the ${ }^{13} \mathrm{C}$ NMR spectrum is indicative of the type of parent skeleton, i.e., spirostanol or furostanol. In the case of spirostanol glycosides, C-22 usually resonates below $\delta \mathrm{c} 110$ and, in the presence of C-23 and C-24 hydroxyls, the C-22 signal is shifted to lower field by 2-4 ppm. In furostanol glycosides having a free hydroxyl or a methoxyl group at C-22, the chemical shift of C-22 is about $\delta \mathrm{c} 111$ in the former case, and $\delta \mathrm{c}$ $112-113$ in the latter $[25,34,35]$. Moreover, furostane saponins do not exhibit IR absorptions at $920-915$ and $900 \mathrm{~cm}^{-1}$ that are characteristic of the spiroketal moiety of a spirostane derivatives. The $920 \mathrm{~cm}^{-1}$ band is more intense than the $900 \mathrm{~cm}^{-1}$ one in (22S)-spirostanes; moreover, an intense band at $900 \mathrm{~cm}^{-1}$, compared to the $920 \mathrm{~cm}^{-1}$ band, is indicative of a $(22 R)$-stereocenter. In addition, the $22 R$ stereochemistry of spirostanes is deduced from the presence of two well separated $\mathrm{H}_{2}-26$ proton signals that appear at $\sim \delta 4.05\left(\mathrm{H}-26_{\text {eq }}\right)$, and $\sim 3.35$ (H-26 $6_{\mathrm{ax}}$ ), respectively.

\subsubsection{Stereochemistry at C- 25}

${ }^{1} \mathrm{H}$ NMR spectra give information on the stereochemistry of the methyl group at C-25, that can occur as $25 R$ - and $25 \mathrm{~S}$ in steroidal saponins. Therefore, the signal of $\mathrm{H}_{3}-27$ usually occurs at $\delta 0.55-0.85$ and $\delta 0.95-1.15$ in (25R)- and (25S)-spirostanes, respectively. Moreover, the difference between the chemical shifts of axial and equatorial protons attached to C-23, C-24, and C-26 can be used to resolve the absolute configuration of C-25 in spirostanes. The differences $\delta \mathrm{eq}-\delta \mathrm{ax}$ for $\mathrm{H}_{2}-23,-24,-26$ are usually $>0.35$ for the $25 S$ configuration, while they are $<0.20 \mathrm{ppm}$ for $(25 R)$ compounds. ${ }^{13} \mathrm{C}$ NMR spectrometry is also very informative about the stereochemistry at C-25. Highly diagnostic are the C-23 and C-25 signals that are significantly moved downfield $\left(\delta_{\mathrm{C}}>30.0\right)$ in $(25 R)$ stereoisomers. NOE and NOESY techniques are also used to determine the stereochemistry at position 25 in spirostanes through the NOE correlations between $\mathrm{H}-25$ and 27-Me with adjacent protons on the ring [31]

For furostanol glycosides, the difference between the chemical shifts of the geminal $\mathrm{H}_{2}-26$ is $<0.48$ for $(25 R)$ and $>0.57$ for $(25 S)$ configuration. Moreover, the methyl group at C-25 resonates at $\delta$ 0.90-1.05 and at $\delta 0.95-1.10$ for (25R)- and (25S)-stereoisomers, respectively [36-39].

The conversion of a furostanol saponin to the corresponding spirostanol form by hydrolysis or enzymatic cleavage of the sugar (usually glucose) moiety at C-26 is still the most reliable method for the prediction of the C-25 configuration in furostanol saponins [24].

\subsubsection{Olefinic Protons and Carbons}

Commonly found olefinic hydrogen $\mathrm{H}-6$ and exomethylene protons $\mathrm{H}_{2}-27$ in spirostane analogues resonate at $\delta 5.25-5.60$ and 4.75-4.85, respectively. Olefinic carbons resonate at $\delta_{\mathrm{C}} 139-140$ (C-5), 121-125 (C-6), 140-145 (C-25), and 108-110 (C-27) in spirostanes and at about $\delta_{\mathrm{C}} 104(\mathrm{C}-20), 152-157(\mathrm{C}-22)$ in the case of furostane analogues.

\subsubsection{Sugar Units}

The protons attached to oxygenated C-1, C-3 and C-16 resonate at $\delta 3.65-3.85$ (dd, J 12 and $4 \mathrm{~Hz}), 3.85-4.05(\mathrm{~m})$, and $4.45-4.56(\mathrm{dd}, J \sim 14.5$ and $8 \mathrm{~Hz})$, respectively. Most of the sugar protons resonate in a narrow range $(\delta 3.0-4.5)$ and are highly overlapped. Using highfield ( $\geq 400 \mathrm{MHz}$ ) NMR instruments the geminal protons of a C-6 oxygenated sugar are usually identifiable as $\mathrm{AB}$ part of a $\mathrm{ABX}$ system $\left(\mathrm{H}_{\mathrm{a}}-6: \delta \sim 4.35-4.45, \mathrm{dd}, J \sim 12\right.$ and $2 \mathrm{~Hz} ; \mathrm{H}_{\mathrm{b}}$ 6: $\delta \sim 4.30-4.35, \mathrm{dd}, J \sim 12$ and $5.5 \mathrm{~Hz}$ ), whereas the $\mathrm{H}_{2}-5$ protons of an $\alpha$-arabinopyranosyl unit give rise to two signals at $\delta \sim 3.65(\mathrm{~d}, J \sim 11 \mathrm{~Hz})$ and $\sim 4.25(\mathrm{~m})$. The anomeric protons are also clearly distinguishable as doublets in the region of $\delta 4.1-6.4$ with coupling constants $\left({ }^{3} \mathrm{~J}\right)$ between $\mathrm{H}-1$ and $\mathrm{H}-2$ in the range of $\sim 7-9 \mathrm{~Hz}$ for a diaxial orientation and $\sim 1-3 \mathrm{~Hz}$, 
for an axial/ equatorial or diequatorial arrangement [40]. Therefore, the $\alpha / \beta$ configuration of the anomeric proton can be inferred from the value of the ${ }^{3} J_{\mathrm{CH}}$ coupling that follows the Karplus relationship. For example, the $\mathrm{H}-1$ of a $\beta$-glucopyranosyl unit usually resonates at $\delta 4.8-5.5$ as a doublet with $J \sim 7-8.5 \mathrm{~Hz}$, whereas the doublet (often broad singlet) for the H-1 of an $\alpha$-rhamnopyranosyl unit usually appears at $\delta 5.7-6.4$ with $J \sim 1.1-1.7 \mathrm{~Hz}$, the signal for the $\mathrm{H}-1$ of an $\alpha$-arabinopyranosyl unit usually resonates as a doublet at $\delta$ 4.6-4.75 with $J \sim 7 \mathrm{~Hz}$, and the doublet of the anomeric proton of a $\beta$-xylopyranosyl unit appears at $\delta 5.10-5.75$ with $J \sim 6-8 \mathrm{~Hz}$ [40]. The $\alpha$-configuration of an $\alpha$-rhamnopyranosyl moiety is also supported by the axial/axial relationship between $\mathrm{H}-3 / \mathrm{H}-4$ and $\mathrm{H}-4 / \mathrm{H}-5$ $(J>9 \mathrm{~Hz})$ and the axial-equatorial $\mathrm{H}-2 / \mathrm{H}-3(J<5 \mathrm{~Hz})$.

In the ${ }^{13} \mathrm{C}$ NMR spectra of saponins, the sugar anomeric carbons resonate in the chemical shift range of $\delta_{C} 96-112$, allowing to infer the number of monosaccharide units present and sometimes also the nature of the glycosidic linkages. The C-22 quaternary acetal carbon also resonates at about $\delta_{\mathrm{C}} 110$. These signals are well differentiated from the other sugar oxygenated carbons that appear in the range of $\delta_{\mathrm{C}} 60-90$, with the carbon of a $\mathrm{CH}_{2} \mathrm{OH}$ group (e.g., C-6 of a glucopyranosyl moiety) resonating at about $\delta_{\mathrm{C}} 62.5$ and that of a $\mathrm{CH}_{2} \mathrm{O}$ group (e.g., C-5 of an arabinopyranosyl moiety) appearing at about $\delta_{\mathrm{C}} 68$. Oxygenated carbons of the sapogenin core occur in the same region as the oxygenated sugar carbons. It is noteworthy that glycosylation causes a downfield shift of 0.5 and 7-12 ppm for the proton and the carbon of a non-anomeric $\mathrm{CHO}$ group involved in a glycosidic linkage, respectively, and an upfield shift of $2-5 \mathrm{ppm}$ for the $\beta$-carbon [41]. Therefore, the terminal monosaccharide does not show any glycosylation shift. These effects are, thus, useful for establishing the glycosylation pattern.

In addition to the information inferred from the 1D NMR spectra, a combination of 2D NMR techniques such as COSY, HOHAHA or TOCSY, HETCOR or HMQC, HMBC, NOESY or ROESY [42-44] are routinely used to establish or confirm the identity of the aglycone, the sugars, and the sugar sequence of the oligosaccharide substituents. Instead, INADEQUATE experiments can rarely be used because they are large sample demanding. Short structural fragments are identified by COSY or HETCOR/HSQC-TOCSY spectra; they are then bonded to each other using the information obtained from NOESY/ROESY and HMBC experiments. NOESY/ROESY spectra also help to establish the configuration of stereocenters. As regards the sugar residues, the spin systems from the anomeric to the terminal proton of each monomer are identified using several 1D-TOCSY and 1DROESY experiments with different mixing times [25]. The ${ }^{13} \mathrm{C}$ sugar signals can then be assigned unambiguously with the help of a HETCOR or HMQC experiment. Finally, NOESY/ROESY and HMBC measurements allow to identify the sugar sequence and the inter-glycosidic bonds. In fact, the presence of an inter-glycosidic NOE from the anomeric proton of a sugar residue to a proton of another sugar or to a sapogenin proton suggests the existence of a glycosidic linkage between the two residues. For example, a NOE correlation between the anomeric proton of the first sugar and the $\mathrm{H}-3$ of aglycone confirms the placement of the oligosaccharide chain at C-3. A ${ }^{3} J_{\mathrm{CH}}$ coupling (HMBC) between the anomeric proton and the aglycone carbon or sugar carbon to which it is linked firmly confirms the glycosidic linkage. For example, in the case of furostanol glycosides, HMBC and ROESY cross peaks between the anomeric proton of a glucopyranosyl moiety and $\mathrm{C}-26$ or $\mathrm{H}_{2}-26$ of the aglycone confirm the glycosylation of $26-\mathrm{OH}$.

Numerous informative examples of the application of these techniques can be found in publications reporting saponins listed in the following tables [26-33].

\section{Steroidal Saponins Isolated from Dracaena and Sansevieria Species}

The steroidal saponins isolated from Dracaena and Sansevieria species are listed in the following tables, in accordance with the number of sugar residues bonded to aglycone. They have been divided between spirostane (Tables 2-5, furostane (Tables 6-8) and miscellaneous saponins (Table 9). The occurrence of saponins in Dracaena and Sansevieria 
species is shown in Table 10, whereas biological properties are reported in Tables 11 and 12.

The chemical structures are shown in Figures 2-9.

Table 2. Spirostanol monoglycosides isolated from Dracaena and Sansevieria species.

\begin{tabular}{|c|c|c|c|}
\hline Number & Compound Name & Plant & References \\
\hline 1 & $\begin{array}{l}\text { (22R)-Spirosta-5,25(27)-diene-1 } \beta, 3 \beta \text {-diol (neoruscogenin) } \\
\text { 1-O- } \alpha \text {-L-arabinopyranoside }\end{array}$ & $\begin{array}{l}\text { D. angustifolia } \\
\text { D. fragrans } \\
\text { (D. deisteliana) } \\
\text { D. thalioides }\end{array}$ & $\begin{array}{l}{[26]} \\
{[27]} \\
{[32]}\end{array}$ \\
\hline 2 & $\begin{array}{c}\text { (22R)-Spirosta-5,25(27)-diene-1 } \beta, 3 \beta \text {-diol (neoruscogenin) } \\
1-O-(4-O-\text {-sulfo)- } \alpha \text {-L-arabinopyranoside (cambodianoside F) }\end{array}$ & $\begin{array}{l}\text { D. cambodiana } \\
\text { D. fragrans } \\
\text { (D. deisteliana) }\end{array}$ & $\begin{array}{l}{[45]} \\
{[46]}\end{array}$ \\
\hline 3 & $\begin{array}{l}(22 R, 25 S) \text {-Spirost-5-ene-1 } \beta, 3 \beta \text {-diol }[(S) \text {-ruscogenin }] \\
\text { 3-O- } \beta \text {-D-glucopyranoside }\end{array}$ & S. cylindrica & [47] \\
\hline 4 & $\begin{array}{c}(22 R, 25 R) \text {-Spirost- } 5 \text {-ene- } 1 \beta, 3 \beta \text {-diol }[(R) \text {-ruscogenin }] \\
1-O-\beta \text {-D-xylopyranoside }\end{array}$ & D. thalioides & [32] \\
\hline 5 & $\begin{array}{c}(22 R, 25 S) \text {-Spirost-5-ene- } 1 \beta, 3 \beta, 7 \beta \text {-triol } \\
1-O-(4-O \text {-sulfo)- } \alpha \text {-L-arabinopyranoside (angudracanoside E) }\end{array}$ & D. angustifolia & {$[48]$} \\
\hline 6 & $(22 R, 25 R)$-Spirost-5-en-3 $\beta$-ol 3-O- $\beta$-D-glucopyranoside (trillin) & $\begin{array}{l}\text { D. marginata } \\
\text { D. viridiflora }\end{array}$ & $\begin{array}{l}{[49]} \\
[50]]\end{array}$ \\
\hline 7 & $\begin{array}{c}\text { (22R,25R)-Spirost-5-ene-3 } \beta, 17 \alpha \text {-diol 3-O- } \beta \text {-D-glucopyranoside } \\
\text { (pennogenin 3-O- } \beta \text {-D-glucopyranoside or floribundasaponin A) }\end{array}$ & $\begin{array}{l}\text { D. arborea } \\
\text { D. draco } \\
\text { D. mannii }\end{array}$ & $\begin{array}{l}{[27]} \\
{[29]} \\
{[51]}\end{array}$ \\
\hline
\end{tabular}

Table 3. Spirostanol diglycosides isolated from Dracaena and Sansevieria species.

\begin{tabular}{|c|c|c|c|}
\hline Number & Compound Name & Plant & References \\
\hline 8 & $\begin{array}{l}(24 S, 25 R)-24-O-\beta \text {-D-Glucopyranosyl-3 } \alpha, 5 \alpha \text {-cyclospirostane- } 1 \beta, 6 \beta, 24 \text {-triol } \\
\text { 1-O- } \beta \text {-D-fucopyranoside }\end{array}$ & D. sarculosa & [52] \\
\hline 9 & $\begin{array}{c}(24 S, 25 R)-24-O-\beta \text {-D-Glucopyranosyl- } 3 \alpha, 5 \alpha \text {-cyclospirostane- } 1 \beta, 6 \beta, 24 \text {-triol } \\
\text { 1-O- } \beta \text {-D-glucopyranoside }\end{array}$ & D. sarculosa & [52] \\
\hline 10 & $\begin{array}{c}(24 S, 25 R)-24-O-\beta-D-G l u c o p y r a n o s y l-s p i r o s t-5 \text {-ene- } 1 \beta, 3 \beta, 24 \text {-triol } \\
\text { 1-O- } \beta \text {-D-fucopyranoside (surculoside B) }\end{array}$ & D. sarculosa & [31] \\
\hline 11 & $\begin{array}{l}\text { (22R)-Spirosta-5,25(27)-diene- } 1 \beta, 3 \beta \text {-diol (neoruscogenin) } \\
\text { 1-O- } \alpha \text {-L-rhamnopyranosyl- }(1 \rightarrow 2)-\alpha \text {-L-arabinopyranoside }\end{array}$ & $\begin{array}{c}\text { D. angustifolia } \\
\text { D. cambodiana } \\
\text { D. draco } \\
\text { D. fragrans } \\
\text { (D. deisteliana) } \\
\text { D. thalioides } \\
\text { S. trifasciata } \\
\text { (D. trifasciata) }\end{array}$ & $\begin{array}{c}{[26]} \\
{[45]} \\
{[29,53]} \\
{[27]} \\
{[32]} \\
{[54]}\end{array}$ \\
\hline 12 & $\begin{array}{l}\text { (22R)-Spirosta-5,25(27)-diene- } 1 \beta, 3 \beta \text {-diol (neoruscogenin) } \\
\text { 1-O- } \alpha \text {-L-rhamnopyranosyl-( } 1 \rightarrow 2 \text { )- } 4-O \text {-sulfo- } \alpha \text {-L-arabinopyranoside } \\
\text { (angudracanoside B }\end{array}$ & $\begin{array}{l}\text { D. angustifolia } \\
\text { D. cambodiana } \\
\text { D. fragrans (D. } \\
\text { deisteliana) }\end{array}$ & $\begin{array}{l}{[48]} \\
{[45]} \\
{[46]}\end{array}$ \\
\hline 13 & $\begin{array}{l}\text { (22R)-Spirosta-5,25(27)-diene- } 1 \beta, 3 \beta, 7 \beta \text {-triol } \\
\text { 1-O- } \alpha \text {-L-rhamnopyranosyl-( } 1 \rightarrow 2)-4-O \text {-sulfo- } \alpha \text {-L-arabinopyranoside } \\
\text { (angudracanoside C) }\end{array}$ & D. angustifolia & [48] \\
\hline 14 & $\begin{array}{c}\text { (22S,23S)-Spirosta-5,25(27)-diene-1 } \beta, 3 \beta, 23 \text {-triol } \\
1-O-\alpha \text {-L-rhamnopyranosyl- }(1 \rightarrow 2) \text { - } \alpha \text {-L-arabinopyranoside }\end{array}$ & D. draco & [53] \\
\hline 15 & $\begin{array}{c}\text { (22S,23S)-Spirosta-5,25(27)-diene- } 1 \beta, 3 \beta, 23 \text {-triol } \\
\text { 1-O-(4-O-acetyl)- } \alpha \text {-L-rhamnopyranosyl- }(1 \rightarrow 2)-\alpha \text {-L-arabinopyranoside }\end{array}$ & D. draco & {$[29,53]$} \\
\hline
\end{tabular}


Table 3. Cont.

\begin{tabular}{|c|c|c|c|}
\hline Number & Compound Name & Plant & References \\
\hline 16 & $\begin{array}{c}(22 R, 24 S) \text {-Spirosta-5,25(27)-diene- } 1 \beta, 3 \beta, 24 \text {-triol } \\
1-O-\alpha \text {-L-rhamnopyranosyl- }(1 \rightarrow 2)-4-O \text {-sulfo- } \alpha \text {-L-arabinopyranoside } \\
\text { (angudracanoside D) }\end{array}$ & D. angustifolia & [48] \\
\hline 17 & $\begin{array}{c}(22 S, 23 S, 24 S) \text {-Spirosta-5,25(27)-diene- } 1 \beta, 3 \beta, 23,24 \text {-tetraol } \\
1-O-\alpha \text {-L-rhamnopyranosyl-( } 1 \rightarrow 2)-O-\alpha \text {-L-arabinopyranoside (draconin B) }\end{array}$ & $\begin{array}{l}\text { D. angustifolia } \\
\text { D. draco }\end{array}$ & $\begin{array}{l}{[26]} \\
{[28,29,53} \\
55]\end{array}$ \\
\hline 18 & $\begin{array}{c}(22 S, 23 S, 24 S) \text {-Spirosta-5,25(27)-diene- } 1 \beta, 3 \beta, 23,24 \text {-tetraol } \\
1-O-\alpha \text {-L-(2-O-acetyl)-rhamnopyranosyl- }(1 \rightarrow 2)-\alpha \text {-L-arabinopyranoside } \\
\text { (draconin } C)\end{array}$ & D. draco & {$[28,29]$} \\
\hline 19 & $\begin{array}{c}(22 S, 23 S, 24 S)-S p i r o s t a-5,25(27) \text {-diene- } 1 \beta, 3 \beta, 23,24 \text {-tetraol } \\
1-O-\alpha \text {-L-(4-O-acetyl)-rhamnopyranosyl- }(1 \rightarrow 2)-\alpha \text {-L-arabinopyranoside } \\
\text { (draconin } C)\end{array}$ & D. draco & {$[28,29,53]$} \\
\hline 20 & $\begin{array}{c}(22 S, 23 S, 24 S)-S p i r o s t a-5,25(27) \text {-diene- } 1 \beta, 3 \beta, 23,24 \text {-tetraol } \\
1-O-\alpha \text {-L-(2,3-di-O-acetyl)-rhamnopyranosyl-( } 1 \rightarrow 2)-\alpha \text {-L-arabinopyranoside } \\
\text { (draconin } B)\end{array}$ & D. draco & {$[28,29]$} \\
\hline 21 & $\begin{array}{c}(22 S, 23 S, 24 S) \text {-Spirosta-5,25(27)-diene- } 1 \beta, 3 \beta, 23,24 \text {-tetraol } \\
\text { 1-O-(2,3,4-tri-O-acetyl)- } \alpha \text {-L-rhamnopyranosyl- }(1 \rightarrow 2)-\alpha \text {-L-arabinopyranoside } \\
\text { (draconin A) }\end{array}$ & D. draco & {$[28,29]$} \\
\hline 22 & $\begin{array}{c}(22 R, 25 R)-5 \beta \text {-Spirostan-3- } \beta \text {-ol (smilagenin) } \\
\text { 3-O- } \beta \text {-D-glucopyranosyl- }(1 \rightarrow 2)-\beta \text {-D-galactopyranoside }\end{array}$ & D. ombet & {$[30]$} \\
\hline 23 & $\begin{array}{l}(22 R, 25 S)-5 \alpha-S p i r o s t a n-3-\beta \text {-ol-12-one } \\
\text { 3-O- } \beta \text {-D-galactopyranosyl- }(1 \rightarrow 4)-\beta \text {-D-glucopyranoside (terreside B) }{ }^{\text {a }}\end{array}$ & D. angustifolia & [48] \\
\hline 24 & $\begin{array}{l}(22 S, 23 S, 25 R)-5 \alpha \text {-Spirostane-3 } \beta, 6 \alpha, 23 \text {-triol-3,6-di-O- } \beta \text {-D-glucopyranoside } \\
\text { (cantalasaponin-1) }\end{array}$ & D. cambodiana & [56] \\
\hline 25 & $\begin{array}{c}(22 R, 25 R)-S p i r o s t-5-e n-3 \beta \text {-ol (diosgenin) } \\
3-O-\alpha \text {-L-rhamnopyranosyl-( } 1 \rightarrow 2)-\beta-D \text {-glucopyranoside (prosapogenin A) }\end{array}$ & $\begin{array}{l}\text { D. draco } \\
\text { D. fragrans } \\
\text { (D. deisteliana) } \\
\text { D. viridiflora } \\
\text { S. ehrenbergii }\end{array}$ & $\begin{array}{l}{[53]} \\
{[46]} \\
{[49]} \\
{[57]}\end{array}$ \\
\hline 26 & $\begin{array}{c}(22 R, 25 R)-S p i r o s t-5-e n-3 \beta \text {-ol (diosgenin) } \\
\text { 3-O- } \alpha \text {-L-rhamnopyranosyl-( } 1 \rightarrow 4)-\beta \text {-D-glucopyranoside (prosapogenin B) }\end{array}$ & $\begin{array}{l}\text { D. draco } \\
\text { D. viridiflora }\end{array}$ & $\begin{array}{c}{[28,55]} \\
{[50]}\end{array}$ \\
\hline 27 & $\begin{array}{c}(22 R, 25 R)-S p i r o s t-5-e n-3 \beta \text {-ol } \\
\text { 3-O-(3-O-sulfo)- } \alpha \text {-L-rhamnosyl-( } 1 \rightarrow 4)-\beta \text {-D-glucopyranoside (deistelianoside A) }\end{array}$ & $\begin{array}{l}\text { D. fragrans } \\
\text { (D. deisteliana) }\end{array}$ & [27] \\
\hline 28 & $\begin{array}{c}(22 R, 25 S) \text {-Spirost-5-ene-1 } \beta, 3 \beta \text {-diol }[(R) \text {-ruscogenin] } \\
1-O-\alpha \text {-L-rhamnopyranosyl-( } 1 \rightarrow 2)-\alpha \text {-L-arabinopyranoside (alliospiroside A) }\end{array}$ & $\begin{array}{l}\text { D. angustifolia } \\
\text { D. concinna } \\
\text { D. marginata } \\
\text { S. cylindrica }\end{array}$ & $\begin{array}{c}{[48,58]} \\
{[59]} \\
{[49]} \\
{[60]}\end{array}$ \\
\hline 29 & $\begin{array}{l}(22 R, 25 R) \text {-Spirost-5-ene- } 1 \beta, 3 \beta \text {-diol }[(R) \text {-ruscogenin }] \\
1-O-\alpha \text {-L-rhamnopyranosyl- }(1 \rightarrow 2)-4 \text { - } O \text {-sulfo- } \alpha \text {-L-arabinopyranoside }\end{array}$ & $\begin{array}{l}\text { D. angustifolia } \\
\text { D. fragrans } \\
\text { (D. deisteliana) }\end{array}$ & $\begin{array}{l}{[48]} \\
{[46]}\end{array}$ \\
\hline 30 & $\begin{array}{c}\text { (22R,25S)-Spirost-5-ene-1 } \beta, 3 \beta \text {-diol }[(S) \text {-ruscogenin] } \\
1-O-\alpha \text {-L-rhamnopyranosyl- }(1 \rightarrow 2)-4 \text {-O-sulfo- } \alpha \text {-L-arabinopyranoside }\end{array}$ & D. marginata & [49] \\
\hline 31 & $\begin{array}{c}\text { (22R,25S)-Spirost-5-ene-1 } \beta, 3 \beta \text {-diol }[(S) \text {-ruscogenin }] \\
1-O \text { - } \alpha \text {-L-rhamnopyranosyl- }(1 \rightarrow 2) \text { - } \beta \text {-D-fucopyranoside }\end{array}$ & D. surculosa & [31] \\
\hline 32 & $\begin{array}{c}(22 R, 25 R) \text {-Spirost-5-ene- } 1 \beta, 3 \beta \text {-diol }[(R) \text {-ruscogenin }] \\
\text { 1-O- } \alpha \text {-L-rhamnopyranosyl-( } 1 \rightarrow 2) \text { - } \beta \text {-D-glucopyranoside }\end{array}$ & $\begin{array}{l}\text { D. marginata } \\
\text { D. thalioides }\end{array}$ & $\begin{array}{l}{[49]} \\
{[32]}\end{array}$ \\
\hline 33 & $\begin{array}{c}\text { (22R,25S)-Spirost-5-ene-1 } \beta, 3 \beta \text {-diol }[(S) \text {-ruscogenin }] \\
\text { 1-O- } \alpha \text {-L-rhamnopyranosyl-( } 1 \rightarrow 2) \text { - } \beta \text {-D-glucopyranoside }\end{array}$ & S. cylindrica & [47] \\
\hline 34 & $\begin{array}{c}(22 R, 25 R) \text {-Spirost-5-ene-1 } \beta, 3 \beta \text {-diol }[(R) \text {-ruscogenin] } \\
1-O-\alpha \text {-L-rhamnopyranosyl- }(1 \rightarrow 2)-\beta \text {-D-xilopyranoside }\end{array}$ & D. thalioides & {$[32]$} \\
\hline
\end{tabular}


Table 3. Cont.

\begin{tabular}{|c|c|c|c|}
\hline Number & Compound Name & Plant & References \\
\hline 35 & $\begin{array}{c}(22 R, 25 S) \text {-Spirost-5-ene-1 } \beta, 3 \beta \text {-diol }[(S) \text {-ruscogenin] } \\
1-O-\beta \text {-D-xylopyranosyl- }(1 \rightarrow 3) \text { - } \alpha \text {-L-arabinopyranoside (angudracanoside } \mathrm{F})\end{array}$ & D. angustifolia & [48] \\
\hline 36 & $\begin{array}{c}(22 R, 25 R) \text {-Spirost-5-ene-1 } \beta, 3 \beta \text {-diol }[(R) \text {-ruscogenin] } \\
\text { 3-O- } \alpha \text {-L-rhamnopyranosyl- }(1 \rightarrow 2)-4 \text { - } O \text {-sulfo- } \alpha \text {-L-arabinopyranoside }\end{array}$ & D. concinna & [59] \\
\hline 37 & $\begin{array}{c}(22 R, 25 S) \text {-Spirost-5-ene- } 1 \beta, 3 \beta \text {-diol }[(S) \text {-ruscogenin }] \\
\text { 3-O- } \alpha \text {-L-rhamnopyranosyl-( } 1 \rightarrow 2)-\beta-D \text {-glucopyranoside (drangustoside B) }\end{array}$ & D. angustifolia & {$[58]$} \\
\hline 38 & $\begin{array}{c}\text { (22R,25S)-Spirost-5-ene-1 } \beta, 3 \beta \text {-diol }[(S) \text {-ruscogenin }] \\
\text { 3-O- } \alpha \text {-L-rhamnopyranosyl-(1 } \rightarrow 4)-\beta \text {-D-glucopyranoside }\end{array}$ & S. cylindrica & [47] \\
\hline 39 & $\begin{array}{l}(22 R, 25 S) \text {-Spirost-5-ene- } 1 \beta, 3 \beta \text {-diol-7-one } \\
\text { 1-O- } \alpha \text {-L-rhamnopyranosyl- }(1 \rightarrow 2)-4-O \text {-sulfo- } \alpha \text {-L-arabinopyranoside } \\
\text { (angudracanoside A) }\end{array}$ & D. angustifolia & [48] \\
\hline 40 & $\begin{array}{c}(22 R, 24 S, 25 R)-S p i r o s t-5 \text {-ene- } 1 \beta, 3 \beta, 24 \text {-triol } \\
1-O-\alpha \text {-L-rhamnopyranosyl- }(1 \rightarrow 2)-\alpha \text {-L-arabinopyranoside (alliospiroside C) }\end{array}$ & D. marginata & [49] \\
\hline 41 & $\begin{array}{c}(22 R, 24 S, 25 S) \text {-Spirost-5-ene- } 1 \beta, 3 \beta, 24 \text {-triol } \\
1-O-\alpha \text {-L-rhamnopyranosyl-( }(\rightarrow 2)-\alpha \text {-L-arabinopyranoside }\end{array}$ & D. marginata & [49] \\
\hline 42 & $\begin{array}{c}(22 R, 25 R)-S p i r o s t-5 \text {-ene-3 } \beta, 7 \alpha \text {-diol } \\
\text { 3-O- } \alpha \text {-L-rhamnopyranosyl-( }(1 \rightarrow 2)-\beta \text {-D-glucopyranoside (sansevierin A) }\end{array}$ & S. ehrenbergii & [57] \\
\hline 43 & $\begin{array}{c}(14 R, 22 R, 25 R \text { and } 14 R, 22 R, 25 S) \text {-Spirost-5-ene-3 } \beta, 14 \text {-diol } \\
\text { 3-O- } \alpha \text {-L-rhamnoyranosyl-( } 1 \rightarrow 2)-\beta \text {-D-glucopyranoside (dracaenoside E) }\end{array}$ & D. cochinchinensis & [61] \\
\hline 44 & $\begin{array}{c}(14 R, 22 R, 25 R \text { and } 14 R, 22 R, 25 S) \text {-Spirost-5-ene-3 } \beta, 14 \text {-diol } \\
\text { 3-O- } \alpha \text {-L-rhamnoyranosyl-( } 1 \rightarrow 4)-\beta \text {-D-glucopyranoside (dracaenoside E) }\end{array}$ & D. cochinchinensis & {$[61]$} \\
\hline 45 & $\begin{array}{l}(17 S, 22 R, 25 R)-S p i r o s t-5 \text {-ene-3 } \beta, 17-d i o l \text { (pennogenin) } \\
\text { 3-O- } \alpha \text {-L-rhamnopyranosyl-(1 } \rightarrow 2) \text { - } \beta \text {-D-glucopyranoside }\end{array}$ & $\begin{array}{l}\text { D. draco } \\
\text { D. surculosa }\end{array}$ & $\begin{array}{l}{[29]} \\
{[31]}\end{array}$ \\
\hline 46 & $\begin{array}{l}(17 S, 22 R, 25 R)-S p i r o s t-5 \text {-ene-3 } \beta, 17-d i o l \text { (pennogenin) } \\
\text { 3-O- } \alpha \text {-L-rhamnopyranosyl-( } 1 \rightarrow 3)-\beta-D \text {-glucopyranoside (mannioside A) }\end{array}$ & $\begin{array}{l}\text { D. arborea } \\
\text { D. mannii } \\
\text { D. thalioides }\end{array}$ & $\begin{array}{l}{[27]} \\
{[51]} \\
{[32]}\end{array}$ \\
\hline 47 & $\begin{array}{l}(17 S, 22 R, 25 R)-S p i r o s t-5 \text {-ene-3 } \beta, 17-\text { diol (pennogenin) } \\
\text { 3-O- } \alpha \text {-L-rhamnopyranosyl-( } 1 \rightarrow 4)-\beta \text {-D-glucopyranoside }\end{array}$ & D. draco & [29] \\
\hline
\end{tabular}

${ }^{a}$ Both terresides A (75) and B (23) are known compounds; in reference [48] authors did not indicate whether one or both saponins were isolated from $D$. angustifolia.

Table 4. Spirostanol triglycosides isolated from Dracaena and Sansevieria species.

\begin{tabular}{|c|c|c|c|}
\hline Number & Compound Name & Plant & References \\
\hline 48 & $\begin{array}{l}(22 R, 24 S, 25 R)-1-O-\beta \text {-D-Fucopyranosyl-spirost-5-ene-1 } \beta, 3 \beta, 24 \text {-triol } \\
\text { 3-O- } \beta \text {-D-apiofuranosyl- }(1 \rightarrow 4)-\beta \text {-D-glucopyranoside (surculoside A) }\end{array}$ & D. sarculosa & [31] \\
\hline 49 & $\begin{array}{c}\text { (22S,23S,24S)-24-O- } \beta \text {-D-Arabinopyranosyl-spirosta-5,25(27)-diene-1 } \beta, 3 \beta, 23,24 \text {-tetrol } \\
\text { 1-O-[(2,3,4-tri-O-acetyl)- } \alpha \text {-L-rhamnopyranosyl- }(1 \rightarrow 2)]-\alpha \text {-L-arabinopyranoside (icodeside) }\end{array}$ & D. draco & [29] \\
\hline 50 & $\begin{array}{c}(22 S, 23 S, 24 S)-24-O-\beta \text {-D-Fucopyranosyl-spirosta-5,25(27)-diene- } 1 \beta, 3 \beta, 23,24 \text {-tetraol } \\
\text { 1-O- } \alpha \text {-L-rhamnopyranosyl-( }(\rightarrow 2) \text { - } \alpha \text {-L-arabinopyranoside (namonin C) }\end{array}$ & $\begin{array}{l}\text { D. angustifolia } \\
\text { D. cambodiana }\end{array}$ & $\begin{array}{c}{[26]} \\
{[26,62]}\end{array}$ \\
\hline 51 & $\begin{array}{c}\text { (22S,23S,24S)-24-O- } \beta \text {-D-Fucopyranosyl-spirosta-5,25(27)-diene-1 } \beta, 3 \beta, 23,24 \text {-tetraol } \\
1-O-[(4-O \text {-acetyl)- } \alpha \text {-L-rhamnopyranosyl- }(1 \rightarrow 2)]-\alpha \text {-L-arabinopyranoside (namonin D) }\end{array}$ & $\begin{array}{l}\text { D. angustifolia } \\
\text { D. cambodiana }\end{array}$ & $\begin{array}{l}{[26]} \\
{[56]}\end{array}$ \\
\hline 52 & $\begin{array}{c}(22 S, 23 S, 24 S)-24-O-\beta \text {-D-Fucopyranosyl-spirosta-5,25(27)-diene-1 } \beta, 3 \beta, 23,24 \text {-tetraol } \\
\text { 1-O-[3,4-O-diacetyl)- } \alpha \text {-L-rhamnopyranosyl- }(1 \rightarrow 2)]-\alpha \text {-L-arabinopyranoside } \\
\text { (cambodracanoside A) }\end{array}$ & D. cambodiana & [56] \\
\hline 53 & $\begin{array}{c}\text { (22S,23S,24S)-24-O- } \beta \text {-D-Fucopyranosyl-spirosta-5,25(27)-diene- } 1 \beta, 3 \beta, 23,24 \text {-tetraol } \\
\text { 1-O-[(2,3,4-O-triacetyl)- } \alpha \text {-L-rhamnopyranosyl- }(1 \rightarrow 2)]-\alpha \text {-L-arabinopyranoside }\end{array}$ & $\begin{array}{l}\text { D. angustifolia } \\
\text { D. draco }\end{array}$ & $\begin{array}{c}{[26]} \\
{[28,53]}\end{array}$ \\
\hline 54 & $\begin{array}{c}(22 S, 23 S, 24 S, 25 R)-24-O-\beta \text {-D-Fucopyranosyl-spirost-5-ene- } 1 \beta, 3 \beta, 23,24 \text {-tetraol } \\
\text { 1-O- } \alpha \text {-L-rhamnopyranosyl- }(1 \rightarrow 2) \text { - } \alpha \text {-L-arabinopyranoside (cambodracanoside B) }\end{array}$ & D. cambodiana & [56] \\
\hline 55 & $\begin{array}{c}(22 R, 24 S, 25 R)-24-O-\beta \text {-D-Glucopyranosyl-spirost-5-en- } 1 b, 3 \beta, 24 \text {-triol } \\
\text { 1-O- } \alpha \text {-L-rhamnopyranosyl-( }(\rightarrow 2)-\beta \text {-D-fucopyranoside (surculoside C) }\end{array}$ & D. sarculosa & [31] \\
\hline
\end{tabular}


Table 4. Cont.

\begin{tabular}{|c|c|c|c|}
\hline Number & Compound Name & Plant & References \\
\hline 56 & $\begin{array}{c}\text { (22R)-Spirosta-5,25(27)-dien-3 } \beta \text {-ol } \\
3-O-\alpha \text {-L-rhamnoyranosyl- }(1 \rightarrow 2)-[\beta \text {-D-glucopyranosyl- }(1 \rightarrow 3)]-\beta \text {-D-glucopyranoside } \\
\text { (dracaenoside } \mathrm{I})\end{array}$ & D. cochinchinensis & [61] \\
\hline 57 & $\begin{array}{c}\text { (22R)-Spirosta-5,25(27)-dien-3 } \beta \text {-ol } \\
\text { 3-O- } \alpha \text {-L-rhamnopyranosyl-( }(1 \rightarrow 2)-[\alpha \text {-L-rhamnopyranosyl- }(1 \rightarrow 4)]-\beta \text {-D-glucopyranoside } \\
\text { (sansevistatin } 1)\end{array}$ & S. ehrenbergii & [57] \\
\hline 58 & $\begin{array}{l}\text { (22R)-Spirosta-5,25(27)-diene-1 } \beta, 3 \beta \text {-diol (neoruscogenin) } \\
1-O-\alpha \text {-L-rhamnopyranosyl- }(1 \rightarrow 2)-[\beta \text {-D-xylopyranosyl- }(1 \rightarrow 3)]-\alpha \text {-L-arabinopyranoside }\end{array}$ & $\begin{array}{l}\text { D. cambodiana } \\
\text { D. fragrans } \\
\text { (D. deisteliana) } \\
\text { D. thalioides } \\
\text { S. trifasciata } \\
\text { (D. trifasciata) }\end{array}$ & $\begin{array}{l}{[45]} \\
{[27]} \\
{[32]} \\
{[33]}\end{array}$ \\
\hline 59 & $\begin{array}{c}\text { (22R)-Spirosta-5,25(27)-diene-1 } \beta, 3 \beta \text {-diol (neoruscogenin) } \\
1-O-\alpha \text {-L-rhamnopyranosyl- }(1 \rightarrow 2)-[\beta \text {-D-xylopyranosyl- }(1 \rightarrow 3)]-\beta-D \text {-glucopyranoside } \\
\text { (trifasciatoside } B)\end{array}$ & $\begin{array}{l}\text { S. trifasciata } \\
\text { (D. trifasciata) }\end{array}$ & [33] \\
\hline 60 & $\begin{array}{c}(22 S, 23 S)-S p i r o s t a-5,25(27) \text {-diene- } 1 \beta, 3 \beta, 23 \text {-triol } \\
1-O-\alpha \text {-L-rhamnopyranosyl- }(1 \rightarrow 2)-[\beta \text {-D-xylopyranosyl- }(1 \rightarrow 3)]-\alpha \text {-L-arabinopyranoside }\end{array}$ & $\begin{array}{l}\text { D. draco } \\
\text { S. trifasciata } \\
\text { (D. trifasciata) }\end{array}$ & $\begin{array}{l}{[53]} \\
{[63]}\end{array}$ \\
\hline 61 & $\begin{array}{c}\text { (22S,23S)-Spirosta-5,25(27)-diene- } 1 \beta, 3 \beta, 23 \text {-triol } 1-O-(2-O \text {-acetyl)- } \alpha \text {-L-rhamnopyranosyl- } \\
(1 \rightarrow 2)-[\beta \text {-D-xylopyranosyl- }(1 \rightarrow 3)]-\alpha \text {-L-arabinopyranoside } \\
\text { (trifasciatoside } \mathrm{K})\end{array}$ & $\begin{array}{l}\text { S. trifasciata } \\
\text { (D. trifasciata) }\end{array}$ & {$[64]$} \\
\hline 62 & $\begin{array}{l}(22 S, 23 S) \text {-Spirosta-5,25(27)-diene- } 1 \beta, 3 \beta, 23 \text {-triol } 1-O-(3-O \text {-acetyl)- } \alpha \text {-L-rhamnopyranosyl- } \\
(1 \rightarrow 2)-[\beta \text {-D-xylopyranosyl- }(1 \rightarrow 3)]-\alpha \text {-L-arabinopyranoside } \\
\text { (trifasciatoside L) }\end{array}$ & $\begin{array}{l}\text { S. trifasciata } \\
\text { (D. trifasciata) }\end{array}$ & {$[64]$} \\
\hline 63 & $\begin{array}{l}(22 S, 23 S) \text {-Spirosta-5,25(27)-diene-1 } \beta, 3 \beta, 23 \text {-triol 1-O-(4-O-acetyl)- } \alpha \text {-L-rhamnopyranosyl- } \\
(1 \rightarrow 2) \text {-[ } \beta \text {-D-xylopyranosyl- }(1 \rightarrow 3)]-\alpha \text {-L-arabinopyranoside }\end{array}$ & $\begin{array}{l}\text { S. trifasciata } \\
\text { (D. trifasciata) }\end{array}$ & {$[33,63]$} \\
\hline 64 & $\begin{array}{c}\text { (22S,23S)-Spirosta-5,25(27)-diene-1 } \beta, 3 \beta, 23 \text {-triol 1-O-(2,3-di-O-acetyl)- } \\
\alpha \text {-L-rhamnopyranosyl- }(1 \rightarrow 2)-[\beta-D \text {-xylopyranosyl- }(1 \rightarrow 3)]-\alpha \text {-L-arabinopyranoside }\end{array}$ & $\begin{array}{l}\text { S. trifasciata } \\
\text { (D. trifasciata) }\end{array}$ & {$[63]$} \\
\hline 65 & $\begin{array}{l}(22 S, 23 S, 24 S)-S p i r o s t a-5,25(27) \text {-diene- } 1 \beta, 3 \beta, 23,24 \text {-tetraol } \\
1-O-\alpha \text {-L-rhamnopyranosyl- }(1 \rightarrow 2)-[\beta \text {-D-xylopyranosyl- }(1 \rightarrow 3)]-\alpha \text {-L-arabinopyranoside }\end{array}$ & $\begin{array}{l}\text { D. cambodiana } \\
\text { S. trifasciata } \\
\text { (D. trifasciata) }\end{array}$ & $\begin{array}{c}{[62]} \\
{[33,63]}\end{array}$ \\
\hline 66 & $\begin{array}{c}(22 S, 23 S, 24 S)-S p i r o s t a-5,25(27) \text {-diene- } 1 \beta, 3 \beta, 23,24-\text { tetraol 1-O-(2-O-acetyl)- } \alpha \text {-L- } \\
\text { rhamnopyranosyl- }(1 \rightarrow 2)-[\beta \text {-D-xylopyranosyl- }(1 \rightarrow 3)]-\alpha \text {-L-arabinopyranoside } \\
\text { (trifasciatoside } M)\end{array}$ & $\begin{array}{l}\text { S. trifasciata } \\
\text { (D. trifasciata) }\end{array}$ & [64] \\
\hline 67 & $\begin{array}{c}(22 S, 23 S, 24 S)-S p i r o s t a-5,25(27) \text {-diene- } 1 \beta, 3 \beta, 23,24-\text { tetraol } 1-O-(3-O-a c e t y l)-\alpha-\mathrm{L}- \\
\text { rhamnopyranosyl- }(1 \rightarrow 2)-[\beta-\mathrm{D} \text {-xylopyranosyl- }(1 \rightarrow 3)]-\alpha \text {-L-arabinopyranoside } \\
\text { (trifasciatoside } \mathrm{N})\end{array}$ & $\begin{array}{l}\text { S. trifasciata } \\
\text { (D. trifasciata) }\end{array}$ & {$[64]$} \\
\hline 68 & $\begin{array}{l}(22 S, 23 S, 24 S)-S p i r o s t a-5,25(27) \text {-diene- } 1 \beta, 3 \beta, 23,24 \text {-tetraol 1-O-(4-O-acetyl)- } \alpha \text {-L- } \\
\text { rhamnopyranosyl- }(1 \rightarrow 2)-[\beta \text {-D-xylopyranosyl- }(1 \rightarrow 3)]-\alpha \text {-L-arabinopyranoside }\end{array}$ & $\begin{array}{l}\text { S. trifasciata } \\
\text { (D. trifasciata) }\end{array}$ & {$[33,63]$} \\
\hline 69 & $\begin{array}{l}(22 S, 23 S, 24 S) \text {-Spirosta-5,25(27)-diene-1 } \beta, 3 \beta, 23,24 \text {-tetraol 1-O-(2,3-di-O-acetyl)- } \alpha \text {-L- } \\
\text { rhamnopyranosyl- }(1 \rightarrow 2)-[\beta \text {-D-xylopyranosyl- }(1 \rightarrow 3)]-\alpha \text {-L-arabinopyranoside }\end{array}$ & $\begin{array}{l}\text { S. trifasciata } \\
\text { (D. trifasciata) }\end{array}$ & {$[33,63]$} \\
\hline 70 & $\begin{array}{c}\text { (22S,23S,24S)-Spirosta-5,25(27)-diene-1 } \beta, 3 \beta, 23,24 \text {-tetraol } 1-O-(2,4 \text {-di-O-acetyl)- } \alpha \text {-L- } \\
\text { rhamnopyranosyl- }(1 \rightarrow 2)-[\beta-\mathrm{D} \text {-xylopyranosyl- }(1 \rightarrow 3)]-\alpha \text {-L-arabinopyranoside } \\
\text { (trifasciatoside } \mathrm{G})\end{array}$ & $\begin{array}{l}\text { S. trifasciata } \\
\text { (D. trifasciata) }\end{array}$ & [33] \\
\hline 71 & $\begin{array}{c}(22 S, 23 S, 24 S) \text {-Spirosta-5,25(27)-diene- } 1 \beta, 3 \beta, 23,24 \text {-tetraol 1-O-(3,4-di-O-acetyl)- } \alpha \text {-L- } \\
\text { rhamnopyranosyl- }(1 \rightarrow 2)-[\beta \text {-D-xylopyranosyl- }(1 \rightarrow 3)]-\alpha \text {-L-arabinopyranoside } \\
\text { (trifasciatoside } H)\end{array}$ & $\begin{array}{l}\text { S. trifasciata } \\
\text { (D. trifasciata) }\end{array}$ & [33] \\
\hline 72 & $\begin{array}{l}(22 S, 23 S, 24 S) \text {-Spirosta-5,25(27)-diene-1 } \beta, 3 \beta, 23,24 \text {-tetraol 1-O-(2,3,4-tri-O-acetyl)- } \alpha \text {-L- } \\
\text { rhamnopyranosyl- }(1 \rightarrow 2) \text {-[ } \beta \text {-D-xylopyranosyl- }(1 \rightarrow 3)]-\alpha \text {-L-arabinopyranoside }\end{array}$ & $\begin{array}{l}\text { D. angustifolia } \\
\text { D. thalioides } \\
\text { S. trifasciata } \\
\text { (D. trifasciata) }\end{array}$ & $\begin{array}{l}{[26]} \\
{[32]} \\
{[63]}\end{array}$ \\
\hline 73 & $\begin{array}{c}(22 R, 25 R)-5 \beta-S p i r o s t a n-3-\beta-o l \text { (smilagenin) } \\
\text { 3-O- } \beta \text {-D-galactopyranosyl- }\left(1^{\prime \prime \prime} \rightarrow 4^{\prime \prime}\right)-\beta-\mathrm{D} \text {-galactopyranosyl- }\left(1^{\prime \prime} \rightarrow 3^{\prime}\right)-\beta \text {-D-glucopyranoside }\end{array}$ & D. ombet & [30] \\
\hline 74 & 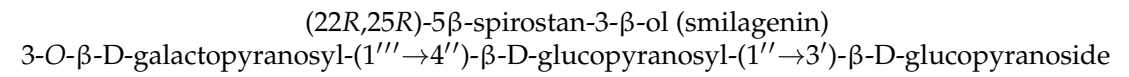 & D. ombet & [30] \\
\hline
\end{tabular}


Table 4. Cont.

\begin{tabular}{|c|c|c|c|}
\hline Number & Compound Name & Plant & References \\
\hline 75 & $\begin{array}{c}(22 R, 25 R)-5 \alpha \text {-Spirostan-3- } \beta \text {-ol- } 12 \text {-one } \\
\text { 3-O- } \beta \text {-D-galactopyranosyl- }\left(1^{\prime \prime \prime} \rightarrow 4^{\prime \prime}\right)-\beta \text {-D-glucopyranosyl- }\left(1^{\prime \prime} \rightarrow 2^{\prime}\right)-\beta \text {-D-glucopyranoside } \\
\text { (terreside A) }{ }^{\text {a }}\end{array}$ & D. angustifolia & [48] \\
\hline 76 & $\begin{array}{c}(22 R, 25 R)-\text { Spirosta- } 3 \beta, 5 \alpha, 6 \beta \text {-triol } \\
\text { 3-O-b-L-rhamnopyranosyl- }(1 \rightarrow 2) \text { - }[\alpha \text {-L-rhamnopyranosyl- }(1 \rightarrow 3)]-\beta \text {-D-glucopyranoside } \\
\text { (cambodianoside } G)\end{array}$ & D. cambodiana & [62] \\
\hline 77 & $\begin{array}{c}(22 R, 25 S) \text {-Spirost-5-ene-1 } \beta, 3 \beta \text {-diol }[(S) \text {-ruscogenin] } \\
\text { 1-O- } \alpha \text {-L-rhamnopyranosyl- }(1 \rightarrow 2)-[\beta \text {-D-xylopyranosyl- }(1 \rightarrow 3)]-\alpha \text {-L-arabinopyranoside }\end{array}$ & $\begin{array}{l}\text { D. angustifolia } \\
\text { D. marginata } \\
\text { D. thalioides } \\
\text { S. cylindrica } \\
\text { S. trifasciata } \\
\text { (D. trifasciata) }\end{array}$ & $\begin{array}{l}{[48]} \\
{[49]} \\
{[32]} \\
{[47]} \\
{[33]}\end{array}$ \\
\hline 78 & $\begin{array}{c}(22 R, 25 R)-\text { Spirost-5-ene- } 1 \beta, 3 \beta \text {-diol }[(R) \text {-ruscogenin] } \\
1-O-\alpha \text {-L-rhamnopyranosyl- }(1 \rightarrow 2)-[\beta \text {-D-xylopyranosyl- }(1 \rightarrow 3)]-\beta \text {-D-glucopyranoside } \\
\text { (trifasciatoside } C)\end{array}$ & $\begin{array}{l}\text { S. trifasciata } \\
\text { (D. trifasciata) }\end{array}$ & [33] \\
\hline 79 & $\begin{array}{c}(22 R, 25 S) \text {-Spirost-5-ene-1b,3 } 3 \text {-diol }[(S) \text {-ruscogenin] } \\
\text { 1-O- } \alpha \text {-L-rhamnopyranosyl- }(1 \rightarrow 2)-[\beta \text {-D-xylopyranosyl- }(1 \rightarrow 3)]-\beta \text {-D-glucopyranoside } \\
\text { (trifasciatoside } D)\end{array}$ & $\begin{array}{l}\text { D. cambodiana } \\
\text { S. trifasciata } \\
\text { (D. trifasciata) }\end{array}$ & $\begin{array}{l}{[45]} \\
{[33]}\end{array}$ \\
\hline 80 & $\begin{array}{c}(22 R, 25 R)-\text { Spirost-5-ene-1 } \beta, 3 \beta \text {-diol }[(R) \text {-ruscogenin] } \\
\text { 1-O- } \alpha \text {-L-rhamnopyranosyl-( } 1 \rightarrow 2) \text {-[ } \beta \text {-D-xylopyranosyl- }(1 \rightarrow 3)]-\beta \text {-D-xylopyranoside }\end{array}$ & D. thalioides & [32] \\
\hline 81 & $\begin{array}{c}(22 R, 24 S, 25 R)-\text { Spirost-5-ene- } 1 \beta, 3 \beta, 24 \text {-triol } \\
\text { 1-O- } \alpha \text {-L-rhamnopyranosyl-(1 } \rightarrow 2)-[\beta \text {-D-xylopyranosyl- }(1 \rightarrow 3)]-\alpha \text {-L-arabinopyranoside }\end{array}$ & D. marginata & [49] \\
\hline 82 & $\begin{array}{c}(22 S, 23 S, 24 S, 25 S) \text {-Spirost-ene-1 } \beta, 3 \beta, 23,24 \text {-tetraol } \\
1-O-\alpha \text {-L-rhamnopyranosyl-( }(1 \rightarrow 2) \text {-[ } \beta \text {-D-xylopyranosyl- }(1 \rightarrow 3)]-\alpha \text {-L-arabinopyranoside }\end{array}$ & D. concinna & [59] \\
\hline 83 & $\begin{array}{l}\text { (22R,25S)-Spirost-5-ene-1 } \beta, 3 \beta \text {-diol }[(S) \text {-ruscogenin] } \\
\text { 3-O- } \alpha \text {-L-rhamnopyranosyl- }(1 \rightarrow 2) \text {-[ } \alpha \text {-L-rhamnopyranosyl- }(1 \rightarrow 3)]-\beta \text {-D-glucopyranoside } \\
\text { (drangustoside A) }\end{array}$ & D. angustifolia & [58] \\
\hline 84 & 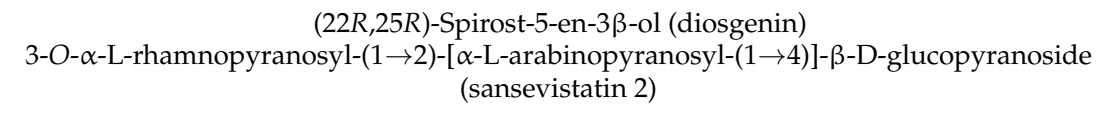 & S. ehrenbergii & [57] \\
\hline 85 & $\begin{array}{l}(22 R, 25 R)-S p i r o s t-5-e n-3 \beta-o l \text { (diosgenin) } \\
\begin{array}{l}\text { 3-O- } \alpha \text {-L-rhamnopyranosyl- }(1 \rightarrow 2)-[\beta-D-\text { glucopyranosyl- }(1 \rightarrow 3)]-\beta-D-g l u c o p y r a n o s i d e \\
\text { (gracillin) }\end{array}\end{array}$ & $\begin{array}{l}\text { D. concinna } \\
\text { D. draco } \\
\text { D. viridiflora }\end{array}$ & $\begin{array}{c}{[,[59]]} \\
{[28]} \\
{[50]}\end{array}$ \\
\hline 86 & $\begin{array}{l}(22 R, 25 R \text { and } 22 R, 25 S) \text {-Spirost-5-en-3 } \beta \text {-ol } \\
\text { 3-O- } \alpha \text {-L-rhamnopyranosyl- }(1 \rightarrow 2)-[\beta \text {-D-glucopyranosyl- }(1 \rightarrow 3)]-\beta \text {-D-glucopyranoside }\end{array}$ & D. cochinchinensis & [61] \\
\hline 87 & $\begin{array}{c}(22 R, 25 R)-S p i r o s t-5-e n-3 \beta \text {-ol (diosgenin) } \\
\text { 3-O- } \alpha \text {-L-rhamnopyranosyl-( }(1 \rightarrow 2) \text {-[ } \alpha \text {-L-rhamnopyranosyl- }(1 \rightarrow 3)]-\beta \text {-D-glucopyranoside }\end{array}$ & D. cambodiana & [45] \\
\hline 88 & $\begin{array}{c}(22 R, 25 R)-\text { Spirost-5-en-3 } \beta \text {-ol (diosgenin) } \\
\text { 3-O- } \alpha \text {-L-rhamnopyranosyl-( }(1 \rightarrow 2)-[\alpha \text {-L-rhamnopyranosyl- }(1 \rightarrow 4)]-\beta \text {-D-glucopyranoside } \\
\text { (dioscin) }\end{array}$ & $\begin{array}{l}\text { D. concinna } \\
\text { D. draco } \\
\text { D. viridiflora } \\
\text { S. ehrenbergii }\end{array}$ & $\begin{array}{l}{[59]} \\
{[28,29,55]} \\
{[50]} \\
{[57]}\end{array}$ \\
\hline 89 & $\begin{array}{l}(22 R, 25 R \text { and } 22 R, 25 S) \text {-Spirost-5-en-3 } \beta \text {-ol } \\
\text { 3-O- } \alpha \text {-L-rhamnopyranosyl- }(1 \rightarrow 2) \text {-[ } \alpha \text {-L-rhamnopyranosyl- }(1 \rightarrow 4)]-\beta \text {-D-glucopyranoside }\end{array}$ & D. cochinchinensis & [61] \\
\hline 90 & $\begin{array}{l}(22 S, 25 S) \text {-Spirost-5-en-3 } \beta \text {-ol } \\
\text { 3-O- } \alpha \text {-L-rhamnopyranosyl-( } 1 \rightarrow 2) \text {-[ } \alpha \text {-L-rhamnopyranosyl- }(1 \rightarrow 4)]-\beta \text {-D-glucopyranoside } \\
\text { (borassoside E) }\end{array}$ & D. marginata & [49] \\
\hline 91 & $\begin{array}{l}(22 R, 25 R) \text {-Spirost-5-en-3 } \beta \text {-ol (diosgenin) } \\
\text { 3-O- } \alpha \text {-L-rhamnopyranosyl- }(1 \rightarrow 2)-[\beta \text {-D-xylopyranosyl-( } 1 \rightarrow 4)]-\beta \text {-D-glucopyranoside }\end{array}$ & S. ehrenbergii & [57] \\
\hline 92 & $\begin{array}{c}(14 R, 22 R, 25 R \text { and } 14 R, 25 S) \text {-Spirost-5-ene-3 } \beta, 14 \text {-diol } \\
\text { 3-O- } \alpha \text {-L-rhamnoyranosyl-( } 1 \rightarrow 2) \text {-[ } \beta \text {-D-glucopyranosyl- }(1 \rightarrow)]-\beta \text {-D-glucopyranoside } \\
(\text { dracaenoside } H)\end{array}$ & D. cochinchinensis & [61] \\
\hline 93 & $\begin{array}{c}(14 R, 22 R, 25 R \text { and } 14 R, 22 R, 25 S) \text {-Spirost-5-ene-3 } \beta, 14 \text {-diol } \\
\text { 3-O- } \alpha \text {-L-rhamnoyranosyl- }(1 \rightarrow 2)-[\alpha \text {-L-rhamnopyranosyl- }(1 \rightarrow 4)]-\beta-D \text {-glucopyranoside } \\
\text { (dracaenoside } G)\end{array}$ & D. cochinchinensis & [61] \\
\hline
\end{tabular}


Table 4. Cont.

\begin{tabular}{|c|c|c|c|}
\hline Number & Compound Name & Plant & References \\
\hline 94 & $\begin{array}{c}(14 R, 22 R, 24 S, 25 R)-S p i r o s t-5 \text {-en-3 } \beta, 14,24 \text {-triol } \\
3-O-\alpha \text {-L-rhamnopyranosyl- }(1 \rightarrow 2)-[\beta \text {-D-glucopyranosyl- }(1 \rightarrow 3)]-\beta \text {-D-glucopyranoside } \\
\text { (dracaenoside } L)\end{array}$ & D. cochinchinensis & {$[61]$} \\
\hline 95 & $\begin{array}{c}(14 R, 22 R, 24 S, 25 R)-S p i r o s t-5 \text {-en-3 } \beta, 14,24 \text {-triol } \\
\text { 3-O- } \alpha \text {-L-rhamnopyranosyl- }(1 \rightarrow 2)-[\alpha \text {-L-rhamnopyranosyl- }(1 \rightarrow 4)]-\beta \text {-D-glucopyranoside } \\
(\text { dracaenoside } \mathrm{K})\end{array}$ & D. cochinchinensis & [61] \\
\hline 96 & $\begin{array}{c}(14 R, 22 R, 25 S) \text {-Spirost-5-ene-3 } \beta, 14,27-\text { triol } \\
\text { 3-O- } \alpha \text {-L-rhamnopyranosyl- }(1 \rightarrow 2)-[\beta \text {-D-glucopyranosyl- }(1 \rightarrow 3)]-\beta \text {-D-glucopyranoside } \\
(\text { dracaenoside })\end{array}$ & D. cochinchinensis & {$[61]$} \\
\hline 97 & $\begin{array}{c}(17 S, 22 R, 25 R)-S p i r o s t-5 \text {-ene-3 } \beta, 17-d i o l \text { (pennogenin) } \\
\text { 3-O- } \alpha \text {-L-rhamnopyranosyl- }(1 \rightarrow 2)-[\alpha \text {-L-rhamnopyranosyl- }(1 \rightarrow 3)]-\beta \text {-D-glucopyranoside } \\
\text { (spiroconazole A) }\end{array}$ & $\begin{array}{l}\text { D. arborea } \\
\text { D. cambodiana } \\
\text { D. mannii } \\
\text { D. thalioides }\end{array}$ & $\begin{array}{c}{[27]} \\
{[45]} \\
{[51,65,66]} \\
{[32]}\end{array}$ \\
\hline 98 & $\begin{array}{c}(17 S, 22 R, 25 R)-S p i r o s t-5 \text {-ene-3 } \beta, 17-\text { diol }(\text { pennogenin }) 3-O-\alpha-L-r h a m n o p y r a n o s y l-(1 \rightarrow 2)-[\alpha-\mathrm{L}- \\
\text { rhamnopyranosyl- }(1 \rightarrow 3)]-(4-O \text {-acetyl)- } \beta \text {-D-glucopyranoside }\end{array}$ & D. thalioides & [32] \\
\hline 99 & $\begin{array}{c}(17 S, 22 R, 25 R)-S p i r o s t-5-e n e-3 \beta, 17-\text { diol }(\text { pennogenin }) 3-O-\alpha \text {-L-rhamnopyranosyl- }(1 \rightarrow 2)-[\alpha-\mathrm{L}- \\
\text { rhamnopyranosyl- }(1 \rightarrow 3)]-(6-O \text {-acetyl })-\beta \text {-D-glucopyranoside } \\
\text { (arboreasaponin } \mathrm{A})\end{array}$ & D. arborea & [27] \\
\hline 100 & $\begin{array}{c}(17 S, 22 R, 25 R)-S p i r o s t-5 \text {-ene-3 } \beta, 17-\text { diol }(\text { pennogenin) } 3-O-\alpha \text {-L-rhamnopyranosyl- }(1 \rightarrow 2)-[\alpha-\mathrm{L}- \\
\text { rhamnopyranosyl- }(1 \rightarrow 3)]-(4,6-O \text {-diacetyl)- } \beta \text {-D-glucopyranoside }\end{array}$ & D. thalioides & [32] \\
\hline 101 & $\begin{array}{c}(17 S, 22 \mathrm{R}, 25 R)-S p i r o s t-5-e n-3 \beta, 17-d i o l \text { (pennogenin) } \\
\text { 3-O- } \alpha \text {-L-rhamnopyranosyl- }(1 \rightarrow 2)-[\alpha \text {-L-rhamnopyranosyl- }(1 \rightarrow 4)]-\beta \text {-D-glucopyranoside } \\
\text { (pennogenin 3-O- } \beta \text {-chacotrioside) }\end{array}$ & $\begin{array}{l}\text { D. draco } \\
\text { D. surculosa }\end{array}$ & $\begin{array}{l}{[29]} \\
{[31]}\end{array}$ \\
\hline 102 & $\begin{array}{c}(17 S, 22 R, 24 R, 25 S) \text {-Spirost-5-ene-3 } \beta, 17,24-\text { triol } \\
\text { 3-O- } \alpha \text {-L-rhamnopyranosyl- }(1 \rightarrow 2)-[\alpha \text {-L-rhamnopyranosyl- }(1 \rightarrow 3)]-\beta \text {-D-glucopyranoside } \\
\text { (arboreasaponin B) }\end{array}$ & D. arborea & [27] \\
\hline 103 & $\begin{array}{c}(22 R, 24 S, 25 S) \text {-Spirost-5-ene-3 } \beta, 24,27-\text { triol } \\
\text { 3-O- } \alpha \text {-L-rhamnopyranosyl- }(1 \rightarrow 2) \text { - }[\alpha \text {-L-rhamnopyranosyl- }(1 \rightarrow 3)]-\beta \text {-D-glucopyranoside } \\
(\text { cambodianoside B) }\end{array}$ & D. cambodiana & [45] \\
\hline 104 & $\begin{array}{c}(22 R, 25 S) \text {-Spirost-5-ene-3 } \beta, 27-\text { diol } \\
\text { 3-O- } \alpha \text {-L-rhamnopyranosyl- }(1 \rightarrow 2)-[\alpha \text {-L-rhamnopyranosyl- }(1 \rightarrow 3)]-\beta \text {-D-glucopyranoside } \\
\text { (cambodianoside } C)\end{array}$ & D. cambodiana & {$[45]$} \\
\hline
\end{tabular}

${ }^{\text {a }}$ Both terresides A (75) and B (23) are known compounds; in reference [48] authors did not indicate whether one or both saponins were isolated from D. angustifolia.

Table 5. Spirostanol tetraglycosides isolated from Dracaena and Sansevieria species.

\begin{tabular}{|c|c|c|c|}
\hline Number & Compound Name & Source & References \\
\hline 105 & $\begin{array}{c}(22 S, 23 S, 24 S)-24-O-\alpha \text {-L-Arabinopyranosyl-spirosta-5,25(27)-diene-1 } \beta, 3 \beta, 23,24 \text {-tetraol } \\
1-O-\beta \text {-D-xylopyranosyl-( } \rightarrow 2) \text {-[ } \alpha \text {-L-rhamnopyranosyl- }(1 \rightarrow 3)]-\beta \text {-D-fucopyranoside } \\
\text { (deistelianoside B) }\end{array}$ & $\begin{array}{l}\text { D. fragrans } \\
\text { (D. deisteliana) }\end{array}$ & [27] \\
\hline 106 & $\begin{array}{l}(22 S, 23 S, 24 S)-24-O-\beta \text {-D-Fucopyranosyl-spirosta-5,25(27)-diene-1 } \beta, 3 \beta, 23,24 \text {-tetraol } \\
\text { 1-O- } \alpha \text {-L-rhamnopyranosyl- }(1 \rightarrow 2)-[\beta \text {-D-xylopyranosyl- }(1 \rightarrow 3)]-O-\alpha \text {-L-arabinopyranoside }\end{array}$ & D. cambodiana & [62] \\
\hline 107 & $\begin{array}{l}(22 S, 23 S, 24 S)-24-O-\beta \text {-D-Fucopyranosyl-spirosta-5,25(27)-diene-1 } \beta, 3 \beta, 23,24 \text {-tetraol 1-O- }(4-O- \\
\text { acetyl)- } \alpha \text {-L-rhamnopyranosyl- }(1 \rightarrow 2)-[\beta-D \text {-xylopyranosyl- }(1 \rightarrow 3)]-\alpha \text {-L-arabinopyranoside }\end{array}$ & $\begin{array}{l}\text { D. cambodiana } \\
\text { S. trifasciata } \\
\text { (D. trifasciata) }\end{array}$ & $\begin{array}{c}{[62]} \\
{[33,63]}\end{array}$ \\
\hline \multirow{2}{*}{108} & \multirow{2}{*}{$\begin{array}{l}(22 S, 23 S, 24 S)-24-O-\beta \text {-D-Fucopyranosyl-spirosta-5,25(27)-diene- } 1 \beta, 3 \beta, 23,24 \text {-tetraol 1-O- }(2,3-\mathrm{di}-\mathrm{O} \text { - } \\
\text { acetyl)- } \alpha \text {-L-rhamnopyranosyl- }(1 \rightarrow 2)-[\beta-\mathrm{D} \text {-xylopyranosyl- }(1 \rightarrow 3)]-\alpha \text {-L-arabinopyranoside }\end{array}$} & D. thalioides & {$[32]$} \\
\hline & & $\begin{array}{l}\text { S. trifasciata } \\
\text { (D. trifasciata) }\end{array}$ & {$[33,63]$} \\
\hline 109 & $\begin{array}{c}(22 S, 23 S, 24 S)-24-O-\beta \text {-D-Fucopyranosyl-spirosta-5,25(27)-diene- } 1 \beta, 3 \beta, 23,24 \text {-tetraol 1-O-(2,4-di-O- } \\
\text { acetyl)- } \alpha \text {-L-rhamnopyranosyl- }(1 \rightarrow 2)-[\beta \text {-D-xylopyranosyl- }(1 \rightarrow 3)]-\alpha \text {-L-arabinopyranoside } \\
\text { (trifasciatoside I) }\end{array}$ & $\begin{array}{l}\text { S. trifasciata } \\
\text { (D. trifasciata) }\end{array}$ & [33] \\
\hline 110 & $\begin{array}{c}(22 S, 23 S, 24 S)-24-O-\beta \text {-D-Fucopyranosyl-spirosta-5,25(27)-diene- } 1 \beta, 3 \beta, 23,24 \text {-tetraol 1-O-(3,4-di-O- } \\
\text { acetyl)- } \alpha \text {-L-rhamnopyranosyl- }(1 \rightarrow 2)-[\beta-D \text {-xylopyranosyl- }(1 \rightarrow 3)]-\alpha \text {-L-arabinopyranoside } \\
\text { (trifasciatoside } J)\end{array}$ & $\begin{array}{l}\text { S. trifasciata } \\
\text { (D. trifasciata) }\end{array}$ & [33] \\
\hline
\end{tabular}


Table 5. Cont.

\begin{tabular}{|c|c|c|c|}
\hline Number & Compound Name & Source & References \\
\hline 111 & $\begin{array}{l}\text { (22S,23S,24S)-24-O- } \beta \text {-D-Fucopyranosyl-spirosta-5,25(27)-diene-1 } \beta, 3 \beta, 23,24 \text {-tetraol 1-O-(2,3,4-tri- } \\
\text { O-acetyl)- } \alpha \text {-L-rhamnopyranosyl-( } 1 \rightarrow 2) \text {-[ } \beta \text {-D-xylopyranosyl- }(1 \rightarrow 3)] \text { - } \alpha \text {-L-arabinopyranoside }\end{array}$ & $\begin{array}{l}\text { D. angustifolia } \\
\text { D. thalioides } \\
\text { S. trifasciata } \\
\text { (D. trifasciata) }\end{array}$ & $\begin{array}{c}{[26]} \\
{[32]} \\
{[33,63]}\end{array}$ \\
\hline 112 & $\begin{array}{c}(22 S, 23 S, 24 S)-24-O-\beta \text {-D-Fucopyranosyl-spirosta-5,25(27)-diene-1 } \beta, 3 \beta, 23,24 \text {-tetraol } \\
\text { 1-O-(2,3,4-tri-O-acetyl)- } \alpha \text {-L-rhamnopyranosyl-( }(\rightarrow 2)-[(3-O \text {-acetyl)- } \beta \text {-D-xylopyranosyl- }(1 \rightarrow 3)]-\alpha- \\
\text { L-arabinopyranoside (namonin A) }\end{array}$ & $\begin{array}{l}\text { D. angustifolia } \\
\text { D. thalioides }\end{array}$ & $\begin{array}{l}{[26]} \\
{[32]}\end{array}$ \\
\hline 113 & $\begin{array}{c}(22 S, 23 S, 24 S)-24-O-\beta \text {-D-Fucopyranosyl-spirosta-5,25(27)-diene-1 } \beta, 3 \beta, 23,24-\text { tetraol } \\
\text { 1-O-(2,3,4-tri-O-acetyl)- } \alpha \text {-L-rhamnopyranosyl- }(1 \rightarrow 2)-[(4-O \text {-acetyl)- } \beta \text {-D-xylopyranosyl-( } 1 \rightarrow 3)]-\alpha \text { - } \\
\text { L-arabinopyranoside (namonin B) }\end{array}$ & D. angustifolia & [26] \\
\hline 114 & $\begin{array}{l}\text { (22S,23S,24S)-24-O- } \beta \text {-D-Glucopyranosyl-spirosta-5,25(27)-diene-1 } \beta, 3 \beta, 23,24 \text {-tetraol 1-O-(2,3,4-tri- } \\
\text { O-acetyl)- } \alpha \text {-L-rhamnopyranosyl-( } 1 \rightarrow 2) \text {-[ } \beta \text {-D-xylopyranosyl- }(1 \rightarrow 3)]-\alpha \text {-L-arabinopyranoside }\end{array}$ & $\begin{array}{l}\text { D. thalioides } \\
\text { S. trifasciata } \\
\text { (D. trifasciata) }\end{array}$ & $\begin{array}{l}{[32]} \\
{[63]}\end{array}$ \\
\hline 115 & $\begin{array}{c}(22 S, 23 S, 24 S)-24-O-\beta-D-G l u c o p y r a n o s y l-s p i r o s t a-5,25(27)-d i e n e-1 \beta, 3 \beta, 23,24-\text { tetraol } \\
1-O-(2,3,4 \text {-tri-O-acetyl)- } \alpha \text {-L-rhamnopyranosyl-( }(1 \rightarrow 2)-[(3-O \text {-acetyl)- } \beta \text {-D-xylopyranosyl- }(1 \rightarrow 3)]-\alpha- \\
\text { L-arabinopyranoside }\end{array}$ & D. thalioides & [32] \\
\hline 116 & $\begin{array}{l}(22 S, 23 S, 24 S)-24-O-\alpha \text {-L-Rhamnopyranosyl-spirosta-5,25(27)-diene-1 } \beta, 3 \beta, 23,24 \text {-tetraol 1-O-(2,3,4- } \\
\text { tri-O-acetyl)- } \alpha \text {-L-rhamnopyranosyl-( }(\rightarrow 2)-[\beta \text {-D-xylopyranosyl- }(1 \rightarrow 3)]-O-\alpha \text {-L-arabinopyranoside }\end{array}$ & $\begin{array}{l}\text { S. trifasciata } \\
\text { (D. trifasciata) }\end{array}$ & [63] \\
\hline 117 & $\begin{array}{l}\text { (22S,23S,24S,25S)-24-O- } \beta \text {-D-Fucopyranosyl-spirost-5-ene- } 1 \beta, 3 \beta, 23,24 \text {-tetraol } \\
\text { 1-O- } \alpha \text {-L-rhamnopyranosyl-( } 1 \rightarrow 2) \text {-[ }[\beta \text {-D-xylopyranosyl- }(1 \rightarrow 3)]-O-\alpha \text {-L-arabinopyranoside }\end{array}$ & D. concinna & [59] \\
\hline 118 & $\begin{array}{l}(22 S, 23 S, 24 S, 25 S)-24-O-\beta \text {-D-Fucopyranosyl-spirost-5-ene-1 } \beta, 3 \beta, 23,24 \text {-tetraol 1-O-(2,3,4-tri-O- } \\
\text { acetyl)- } \alpha \text {-L-rhamnopyranosyl- }(1 \rightarrow 2) \text {-[ } \beta \text {-D-xylopyranosyl- }(1 \rightarrow 3)]-O-\alpha \text {-L-arabinopyranoside }\end{array}$ & D. thalioides & [32] \\
\hline
\end{tabular}

Table 6. Furostanol diglycosides isolated from Dracaena and Sansevieria species.

\begin{tabular}{|c|c|c|c|}
\hline Number & Compound Name & Source & References \\
\hline 119 & $\begin{array}{c}\text { (22S,25S)-Furost-5-en-22(25)-epoxy-1 } \beta, 3 \beta, 26 \text {-triol } \\
\text { 26-O- } \beta \text {-D-glucopyranosyl-( } 1 \rightarrow 2)-\beta \text {-D-glucopyranoside } \\
\text { (cambodianoside E) }\end{array}$ & D. cambodiana & [45] \\
\hline 120 & $\begin{array}{c}(22 R, 25 S)-26-O-\beta-D-G l u c o p y r a n o s y l-22-\text { methoxy- } 3 \alpha, 5 \alpha- \\
\text { cyclofurostane-1 } \beta, 6 \beta, 26 \text {-triol } \\
1-O-\beta \text {-D-fucopyranoside }\end{array}$ & D. surculosa & [52] \\
\hline 121 & $\begin{array}{c}(22 R, 25 S)-26-O-\beta-D-G l u c o p y r a n o s y l-22-\text { methoxy- } 3 \alpha, 5 \alpha- \\
\text { cyclofurostane-1 } \beta, 6 \beta, 26 \text {-triol } \\
\text { 1-O- } \beta \text {-D-glucopyranoside }\end{array}$ & D. surculosa & [52] \\
\hline 122 & $\begin{array}{c}(22 R, 25 S)-26-O-\beta-D-G l u c o p y r a n o s y l-22-m e t h o x y-f u r o s t-5 \text {-ene- } \\
1 \beta, 3 \beta, 26 \text {-triol } \\
\text { 1-O- } \beta \text {-D-fucopyranoside }\end{array}$ & D. surculosa & [31] \\
\hline 123 & $\begin{array}{c}(22 R, 25 S)-26-O-\beta \text {-D-Glucopyranosyl-22-methoxy-furost-5-ene- } \\
1 \beta, 3 \beta, 26 \text {-triol } \\
\text { 1-O- } \beta \text {-D-glucopyranoside }\end{array}$ & D. surculosa & {$[31]$} \\
\hline
\end{tabular}


Table 7. Furostanol triglycosides isolated from Dracaena and Sansevieria species.

\begin{tabular}{|c|c|c|c|}
\hline Number & Compound Name & Source & References \\
\hline 124 & $\begin{array}{c}(22 R, 25 R) \text {-Furost-5-ene-3 } \beta, 22,26-\text { triol } 3-O-\alpha-\mathrm{L}-\text { rhamnopyranosyl- }(1 \rightarrow 2)-[\beta-\mathrm{D} \text { - } \\
\text { glucopyranosyl-( } 1 \rightarrow 3)]-\beta \text {-D-glucopyranoside } \\
\text { (icogenin) }\end{array}$ & D. draco & [55] \\
\hline 125 & $\begin{array}{c}(14 R, 22 S, 25 S)-F u r o s t-5 \text {-en-22(25)-epoxy-3 } \beta, 14,26,27 \text {-tetraol 3-O- } \alpha \text {-L- } \\
\text { rhamnopyranosyl-( }(1 \rightarrow 2) \text {-[ } \alpha \text {-L-rhamnopyranosyl- }(1 \rightarrow 4)]-\beta \text {-D-glucopyranoside } \\
\text { (dracaenoside R) }\end{array}$ & D. cochinchinensis & {$[61]$} \\
\hline 126 & $\begin{array}{l}\text { (22R)-26-O- } \beta \text {-D-Glucopyranosyl-furosta-5,25(27)-diene-1 } \beta, 3 \beta, 22,26 \text {-tetraol } \\
\text { 1-O- } \alpha \text {-L-rhamnopyranosyl- }(1 \rightarrow 2)-\alpha \text {-L-arabinopyranoside }\end{array}$ & $\begin{array}{l}\text { S. trifasciata } \\
\text { (D. trifasciata) }\end{array}$ & {$[33]$} \\
\hline 127 & $\begin{array}{l}\text { (22R)-26-O- } \beta \text {-D-Glucopyranosyl-furosta-5,20(22),25(27)-triene- } 1 \beta, 3 \beta, 22,26 \text {-tetraol } \\
\text { 1-O- } \alpha \text {-L-rhamnopyranosyl- }(1 \rightarrow 2)-\alpha \text {-L-arabinopyranoside }\end{array}$ & $\begin{array}{l}\text { D. angustifolia } \\
\text { D. cambodiana }\end{array}$ & [26] \\
\hline 128 & $\begin{array}{c}(22 R, 25 S)-26-O-\beta \text {-D-Glucopyranosyl-furost-5-ene- } 1 \beta, 3 \beta, 22,26 \text {-tetraol } \\
\text { 1-O- } \alpha \text {-L-rhamnopyranosyl- }(1 \rightarrow 2) \text { - } \alpha \text {-L-arabinopyranoside (alliofuroside A) }\end{array}$ & D. marginata & [49] \\
\hline 129 & $\begin{array}{l}(22 R, 25 S)-26-O-\beta \text {-D-Glucopyranosyl-furost-5-ene- } 1 \beta, 3 \beta, 22,26 \text {-tetraol } \\
1-O-\alpha \text {-L-rhamnopyranosyl-( } 1 \rightarrow 2)-(4-O \text {-sulfo)- } \alpha \text {-L-arabinopyranoside }\end{array}$ & D. marginata & [49] \\
\hline 130 & $\begin{array}{c}(22 R, 25 S)-26-O-\beta \text {-D-Glucopyranosyl-3-O-sulfo-furost-5-ene- } 1 \beta, 3 \beta, 22,26 \text {-tetraol } \\
\text { 1-O- } \alpha \text {-L-rhamnopyranosyl-( } 1 \rightarrow 2)-(3,4-O \text {-diacetyl)- } \alpha \text {-L-arabinopyranoside sodium } \\
\text { salt }\end{array}$ & D. thalioides & [67] \\
\hline 131 & $\begin{array}{c}(14 R, 22 \xi, 25 R+ \\
14 R, 22 \xi, 25 S)-26-O-\beta-D-G l u c o p y r a n o s y l-f u r o s t-5-e n e-3 \beta, 14,22,26-\text { tetraol } \\
\text { 3-O- } \alpha \text {-L-rhamnopyranosyl-(1 } \rightarrow 2)-\beta \text {-D-glucopyranoside (ophipojaponin A + } \\
\text { dracaenoside } \mathrm{N})\end{array}$ & D. cochinchinensis & {$[61]$} \\
\hline 132 & $\begin{array}{c}(14 R, 22 \xi, 25 R \text { and } \\
14 R, 22 \xi, 25 S)-26-O-\beta-D-G l u c o p y r a n o s y l-f u r o s t-5 \text {-ene-3 } \beta, 14,22,26-\text { tetraol } \\
\text { 3-O- } \alpha \text {-L-rhamnopyranosyl-( }(1 \rightarrow 4)-\beta \text {-D-glucopyranoside (dracaenoside } M)\end{array}$ & D. cochinchinensis & [61] \\
\hline 133 & 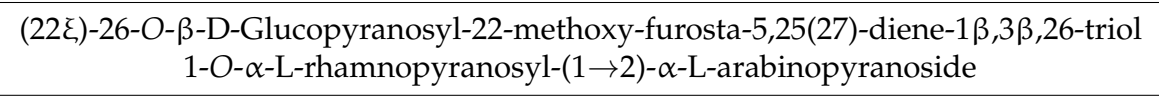 & $\begin{array}{l}\text { D. cambodiana } \\
\text { D. draco }\end{array}$ & $\begin{array}{l}{[56]} \\
{[53]}\end{array}$ \\
\hline 134 & $\begin{array}{l}(22 R)-26-O-\beta \text {-D-Glucopyranosyl-22-methoxy-furosta-5,25(27)-diene- } 1 \beta, 3 \beta, 26 \text {-triol } \\
\text { 1-O- } \alpha \text {-L-rhamnopyranosyl-( } 1 \rightarrow 2) \text { - } \alpha \text {-L-arabinopyranoside }\end{array}$ & $\begin{array}{l}\text { S. trifasciata } \\
\text { (D. trifasciata) }\end{array}$ & [63] \\
\hline 135 & $\begin{array}{c}(22 R, 25 S)-26-O-\beta \text {-D-Glucopyranosyl-22-methoxy-furost-5-ene- } 1 \beta, 3 \beta, 26 \text {-triol } \\
\text { 1-O- } \alpha \text {-L-rhamnopyranosyl- }(1 \rightarrow 2)-\beta \text {-D-fucopyranoside }\end{array}$ & D. surculosa & [31] \\
\hline 136 & 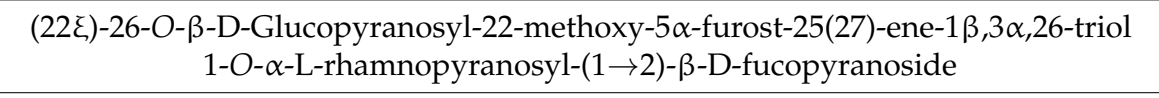 & D. concinna & [59] \\
\hline 137 & 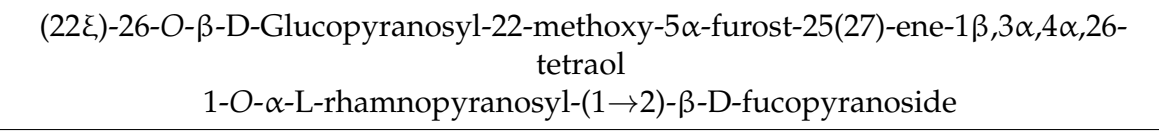 & D. concinna & [59] \\
\hline 138 & 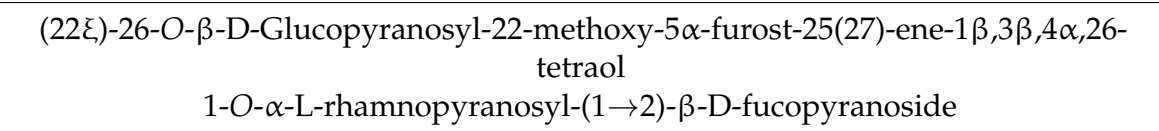 & D. concinna & [59] \\
\hline 139 & $\begin{array}{l}\text { (22R,25R)-26-O- } \alpha \text {-L-Rhamnopyranosyl-furost-5-ene-3 } \beta, 22,26 \text {-triol } \\
\text { 3-O- } \alpha \text {-L-rhamnopyranosyl-( } \rightarrow 4 \text { - } \beta \text {-D-glucopyranoside (afromontoside) }\end{array}$ & D. afromontana & [68] \\
\hline
\end{tabular}


Table 8. Furostanol tetraglycosides isolated from Dracaena and Sansevieria species.

\begin{tabular}{|c|c|c|c|}
\hline Number & Compound Name & Source & References \\
\hline 140 & $\begin{array}{c}\text { (25R)-26-O- } \beta \text {-D-Glucopyranosyl-furosta-5,20(22)-diene- } 1 \beta, 3 \beta, 26 \text {-triol } \\
\text { 1-O- } \alpha \text {-L-rhamnopyranosyl-( }(1 \rightarrow 2)-[\beta \text {-D-xylopyranosyl- }(1 \rightarrow 3)]-(4-\mathrm{O} \text {-acetyl)- } \alpha \text {-L- } \\
\text { arabinopyranoside (namonin } \mathrm{E})\end{array}$ & D. angustifolia & [26] \\
\hline 141 & 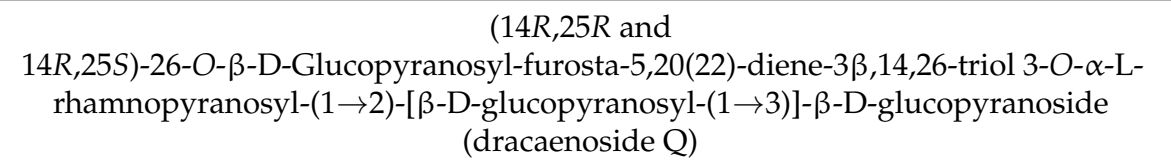 & D. cochinchinensis & [61] \\
\hline 142 & $\begin{array}{c}\text { (22R)-26-O- } \beta \text {-D-Glucopyranosyl-furosta-5,25(27)-diene- } 1 \beta, 3 \beta, 22,26 \text {-tetraol } \\
\text { 1-O-(4-O-acetyl)- } \alpha \text {-L-rhamnopyranosyl- }(1 \rightarrow 2)-[\beta-\mathrm{D} \text {-xylopyranosyl- }(1 \rightarrow 3)]-\alpha \text { - } \\
\text { L-arabinopyranoside }\end{array}$ & $\begin{array}{l}\text { S. trifasciata } \\
\text { (D. trifasciata) }\end{array}$ & {$[33]$} \\
\hline 143 & 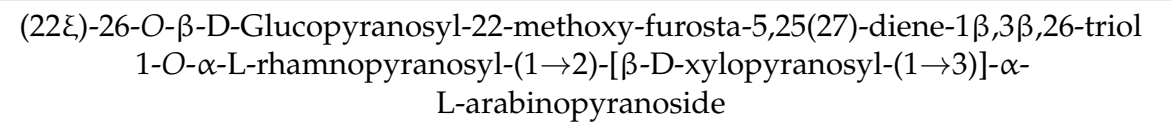 & D. angustifolia & {$[48]$} \\
\hline 144 & 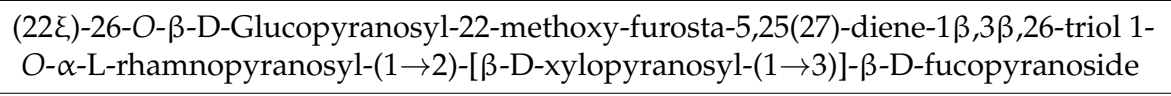 & D. concinna & [59] \\
\hline 145 & $\begin{array}{c}(22 S, 25 S)-26-O-\beta \text {-D-Glucopyranosyl-furost-5-en-22(25)-epoxy-1 } \beta, 3 \beta, 26 \text {-triol 1-O- } \\
\alpha \text {-L-rhamnopyranosyl- }(1 \rightarrow 2)-[\beta \text {-D-xylopyranosyl- }(1 \rightarrow 3)]-\beta \text {-D-glucopyranoside } \\
\text { (trifasciatoside } A)\end{array}$ & $\begin{array}{l}\text { S. trifasciata } \\
\text { (D. trifasciata) }\end{array}$ & [33] \\
\hline 146 & $\begin{array}{l}(22 R, 25 R)-26-O-\beta \text {-D-Glucopyranosyl-furost-5-ene- } 1 \beta, 3 \beta, 22,26 \text {-tetraol 1-O- } \alpha \text {-L- } \\
\text { rhamnopyranosyl- }(1 \rightarrow 2) \text {-[ } \beta \text {-D-xylopyranosyl- }(1 \rightarrow 3)] \text { - } \alpha \text {-L-arabinopyranoside }\end{array}$ & S. cylindrica & [47] \\
\hline 147 & $\begin{array}{l}(22 R, 25 S)-26-O-\beta \text {-D-Glucopyranosyl-furost-5-ene- } 1 \beta, 3 \beta, 22,26 \text {-tetraol } 1-O-\alpha \text {-L- } \\
\text { rhamnopyranosyl- }(1 \rightarrow 2) \text { - }[\beta \text {-D-xylopyranosyl- }(1 \rightarrow 3)]-\alpha \text {-L-arabinopyranoside }\end{array}$ & D. thalioides & [67] \\
\hline $147 a^{a}$ & $\begin{array}{l}\text { 26-O- } \beta \text {-D-Glucopyranosyl-furost-5-ene- } 1 \beta, 3 \beta, 22,26 \text {-tetraol 1-O- } \alpha \text {-L- } \\
\text { rhamnopyranosyl- }(1 \rightarrow 2)-[\beta \text {-D-xylopyranosyl- }(1 \rightarrow 3)]-\alpha \text {-L-arabinopyranoside }\end{array}$ & D. angustifolia & [48] \\
\hline 148 & $\begin{array}{c}(22 R, 25 S)-26-O-\beta-D-G l u c o p y r a n o s y l-f u r o s t-5-e n e-1 \beta, 3 \beta, 22,26-\text { tetraol } 1-O-\alpha \text {-L- } \\
\text { rhamnopyranosyl- }(1 \rightarrow 2)-[\beta \text {-D-xylopyranosyl- }(1 \rightarrow 3)]-\beta \text {-D-glucopyranoside } \\
\text { (trifasciatoside E) }\end{array}$ & $\begin{array}{l}\text { S. trifasciata } \\
\text { (D. trifasciata) }\end{array}$ & [33] \\
\hline 149 & $\begin{array}{l}(22 R, 25 R)-26-O-\beta \text {-D-Glucopyranosyl-furost-5-ene- } 1 \beta, 3 \beta, 22,26 \text {-tetraol 1-O- } \alpha \text {-L- } \\
\text { rhamnopyranosyl- }(1 \rightarrow 2) \text {-[ } \beta \text {-D-xylopyranosyl- }(1 \rightarrow 3)]-\beta \text {-D-xylopyranoside }\end{array}$ & D. thalioides & [67] \\
\hline 150 & $\begin{array}{l}(22 R, 25 S)-26-O-\beta \text {-D-Glucopyranosyl-furost-5-ene- } 1 \beta, 3 \beta, 22,26 \text {-tetraol } 1-O-\alpha \text {-L- } \\
\text { rhamnopyranosyl-( }(\rightarrow 2) \text {-[ } \alpha \text {-L-rhamnopyranosyl- }(1 \rightarrow 4)]-\beta \text {-D-glucopyranoside }\end{array}$ & D. marginata & [49] \\
\hline 151 & $\begin{array}{l}(22 R, 25 S)-26-O-\beta \text {-D-Glucopyranosyl-22-methoxy-furost-5-ene- } 1 \beta, 3 \beta, 26 \text {-triol 1-O- } \\
\alpha \text {-L-rhamnopyranosyl- }(1 \rightarrow 2)-[\beta \text {-D-xylopyranosyl- }(1 \rightarrow 3)]-\beta \text {-D-glucopyranoside } \\
\text { (trifasciatoside } \mathrm{F})\end{array}$ & $\begin{array}{l}\text { S. trifasciata } \\
\text { (D. trifasciata) }\end{array}$ & [33] \\
\hline 152 & $\begin{array}{c}(12 R, 15 R, 22 R, 25 S)-26-O-\beta-D-G l u c o p y r a n o s y l-12,15-d i-O-\alpha \text {-L-rhamnopyranosyl- } \\
\text { furost-5-ene-3 } \beta, 12,15,22,26 \text {-pentaol } \\
\text { 3-O- } \beta \text {-D-glucopyranoside }\end{array}$ & S. cylindrica & [69] \\
\hline 153 & 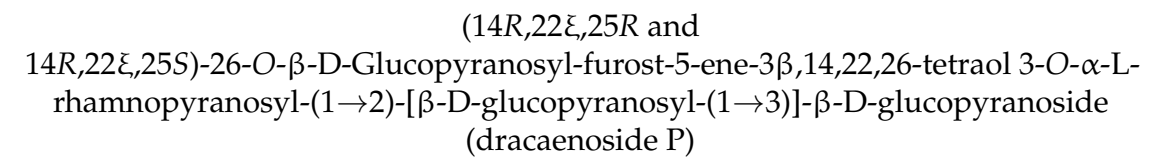 & D. cochinchinensis & {$[61]$} \\
\hline 154 & 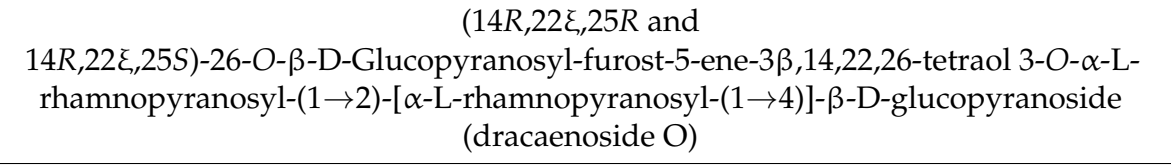 & D. cochinchinensis & {$[61]$} \\
\hline 155 & $\begin{array}{l}(22 R, 25 R)-26-O-\beta \text {-D-Glucopyranosyl-furost-5-ene-3 } \beta, 22,26 \text {-triol 3-O- } \alpha \text {-L- } \\
\text { rhamnopyranosyl- }(1 \rightarrow 2)-[\beta \text {-D-glucopyranosyl- }(1 \rightarrow 3)]-\beta \text {-D-glucopyranoside }\end{array}$ & D. draco & [28] \\
\hline 156 & 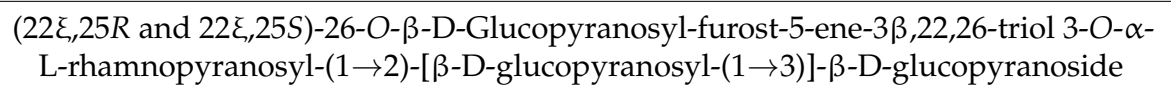 & D. cochinchinensis & [61] \\
\hline
\end{tabular}


Table 8. Cont.

\begin{tabular}{|c|c|c|c|}
\hline Number & Compound Name & Source & References \\
\hline 157 & $\begin{array}{l}(22 R, 25 R)-26-O-\beta \text {-D-Glucopyranosyl-furost-5-ene-3 } \beta, 22,26 \text {-triol 3-O- } \alpha \text {-L- } \\
\text { rhamnopyranosyl- }(1 \rightarrow 2) \text {-[ } \alpha \text {-L-rhamnopyranosyl- }(1 \rightarrow 4)]-\beta \text {-D-glucopyranoside }\end{array}$ & D. draco & [28] \\
\hline 158 & $\begin{array}{l}(22 R, 25 S)-26-O-\beta \text {-D-Glucopyranosyl-furost- } 5 \text {-ene- } 3 \beta, 22,26-\text { triol } \\
\text { 3-O- } \alpha \text {-L-rhamnopyranosyl- }(1 \rightarrow 2) \text { - }[\alpha \text {-L-rhamnopyranosyl- }(1 \rightarrow 4)]-\beta \text {-D- } \\
\text { glucopyranoside (protoneodioscin) }\end{array}$ & D. marginata & [49] \\
\hline 159 & $\begin{array}{l}(22 \xi, 25 R \text { and } 22 \xi, 25 S)-26-O-\beta \text {-D-Glucopyranosyl-furost-5-ene-3 } \beta, 22,26 \text {-triol 3-O- } \alpha \text { - } \\
\text { L-rhamnopyranosyl- }(1 \rightarrow 2) \text {-[ } \alpha \text {-L-rhamnopyranosyl- }(1 \rightarrow 4)]-\beta \text {-D-glucopyranoside }\end{array}$ & D. cochinchinensis & {$[61]$} \\
\hline 160 & $\begin{array}{l}(22 \xi, 25 R)-26-O-\beta \text {-D-Glucopyranosyl-22-methoxy-furost-5-ene-3 } \beta, 26-\text { diol 3-O- } \alpha \text {-L- } \\
\text { rhamnopyranosyl-( }(1 \rightarrow 2) \text {-[ } \beta \text {-D-glucopyranosyl- }(1 \rightarrow 3)]-\beta \text {-D-glucopyranoside }\end{array}$ & D. concinna & [59] \\
\hline 161 & $\begin{array}{c}(22 R, 25 R)-26-O-\beta-\mathrm{D}-\text { Glucopyranosyl-22-methoxy-furost-5-ene-3 } \beta, 26-\text { diol } 3-O-\alpha-\mathrm{L}- \\
\text { rhamnopyranosyl-( }(1 \rightarrow 2)-[\alpha \text {-L-rhamnopyranosyl- }(1 \rightarrow 4)]-\beta \text {-D-glucopyranoside } \\
\text { (methyl protodioscin) }\end{array}$ & D. viridiflora & {$[50]$} \\
\hline 162 & $\begin{array}{c}\text { (22R,25S)-26-O- } \beta \text {-D-Glucopyranosyl-22-methoxy-furost-5-ene-3 } \beta, 26 \text {-diol 3-O- } \alpha \text {-L- } \\
\text { rhamnopyranosyl- }(1 \rightarrow 2) \text {-[ } \alpha \text {-L-rhamnopyranosyl- }(1 \rightarrow 4)]-\beta \text {-D-glucopyranoside } \\
\text { (methyl protoneodioscin) }\end{array}$ & D. marginata & [49] \\
\hline 163 & $\begin{array}{l}(22 \xi, 25 R)-26-O-\beta \text {-D-Glucopyranosyl-22-methoxy-furost-5-ene-3 } \beta, 26 \text {-diol 3- } O \text { - } \alpha \text {-L- } \\
\text { rhamnopyranosyl- }(1 \rightarrow 2) \text {-[ } \alpha \text {-L-rhamnopyranosyl- }(1 \rightarrow 4)]-\beta-D \text {-glucopyranoside }\end{array}$ & D. concinna & [59] \\
\hline
\end{tabular}

a Unassigned stereochemistry at C-22 and C-25.

Table 9. Miscellaneous steroidal saponins isolated from Dracaena and Sansevieria species.

\begin{tabular}{|c|c|c|c|}
\hline Number & Compound Name & Source & References \\
\hline 164 & Cambodianoside A & D. cambodiana & [45] \\
\hline 165 & Cambodianoside D & D. cambodiana & [45] \\
\hline 166 & Dracaenoside A & D. cochinchinensis & {$[70]$} \\
\hline 167 & Dracaenoside B & D. cochinchinensis & [70] \\
\hline 168 & $\begin{array}{c}\text { Pregna-5,16-diene- } 1 \beta, 3 \beta \text {-diol-20-one } \\
\text { 1-O- } \alpha \text {-L-rhamnopyranosyl- }(1 \rightarrow 2)-\alpha \text {-L-arabinopyranoside }\end{array}$ & $\begin{array}{l}\text { D. angustifolia } \\
\text { D. cambodiana } \\
\text { S. trifasciata } \\
\text { (D. trifasciata) }\end{array}$ & $\begin{array}{l}{[26]} \\
{[62]} \\
{[71]}\end{array}$ \\
\hline 169 & $\begin{array}{l}\text { Pregna-5,16-diene-1 } \beta, 3 \beta \text {-diol-20-one } 1-O-\alpha \text {-L-rhamnopyranosyl- }(1 \rightarrow 2)-[\beta \text {-D- } \\
\text { xylopyranosyl- }(1 \rightarrow 3)]-\alpha \text {-L-arabinopyranoside }\end{array}$ & $\begin{array}{l}\text { S. trifasciata } \\
\text { (D. trifasciata) }\end{array}$ & {$[33,71]$} \\
\hline 170 & $\begin{array}{c}\text { Pregna-5,16-diene-1 } \beta, 3 \beta \text {-diol-20-one } 1-O-\alpha \text {-L-rhamnopyranosyl- }(1 \rightarrow 2)-[\beta \text {-D- } \\
\text { xylopyranosyl- }(1 \rightarrow 3)]-\beta \text {-D-glucopyranoside }\end{array}$ & $\begin{array}{l}\text { S. trifasciata } \\
\text { (D. trifasciata) }\end{array}$ & {$[33,71]$} \\
\hline 171 & $\begin{array}{l}\text { Pregna-5,16-diene-1 } \beta, 3 \beta \text {-diol-20-one } 1-O-\alpha \text {-L-rhamnopyranosyl- }(1 \rightarrow 2)-[\beta \text {-D- } \\
\text { xylopyranosyl- }(1 \rightarrow 3)]-6-O \text {-acetyl- } \beta \text {-D-glucopyranoside }\end{array}$ & $\begin{array}{l}\text { S. trifasciata } \\
\text { (D. trifasciata) }\end{array}$ & [71] \\
\hline 172 & $\begin{array}{l}\text { Pregna-5,16-diene-3 } \beta, 14 \alpha \text {-diol-20-one 3-O- } \alpha \text {-L-rhamnopyranosyl-( } 1 \rightarrow 2) \text {-[ } \beta \text {-D- } \\
\text { glucopyranosyl-( }(1 \rightarrow 3)]-\beta \text {-D-glucopyranoside (dracaenoside } \mathrm{D})\end{array}$ & D. cochinchinensis & {$[72]$} \\
\hline 173 & $\begin{array}{l}\text { Pregna-5,16-dien-3 } \beta \text {-ol-20-one } 3-O-\alpha \text {-L-rhamnopyranosyl- }(1 \rightarrow 2)-[\alpha \text {-L- } \\
\text { rhamnopyranosyl- }(1 \rightarrow 3)]-\beta \text {-D-glucopyranoside }\end{array}$ & D. cambodiana & [45] \\
\hline 174 & $\begin{array}{l}\text { Pregna-5,16-diene-3 } \beta, 14 \alpha \text {-diol-20-one 3-O- } \alpha \text {-L-rhamnopyranosyl- }(1 \rightarrow 2) \text {-[ } \alpha \text {-L- } \\
\text { rhamnopyranosyl- }(1 \rightarrow 4)]-\beta \text {-D-glucopyranoside (dracaenoside } \mathrm{C})\end{array}$ & D. cochinchinensis & [72] \\
\hline 175 & $\begin{array}{l}\text { (16S)-1-O- } \alpha \text {-L-Rhamnopyranosyl-( } 1 \rightarrow 2)-O-\alpha \text {-L-arabinopyranosyl-pregn-5-ene- } \\
1 \beta, 3 \beta, 16 \text {-triol 16-O-(4- } \beta \text {-D-glucopyranosyloxymethyl)-pent-4-enoate } \\
\text { (namonin F) }\end{array}$ & D. angustifolia & [26] \\
\hline 176 & $\begin{array}{l}(25 R)-26-O-\beta \text {-D-Glucopyranoyl-cholesta-5,17-dien-3 } \beta \text {-ol-16,22-dione } 3-O-\alpha \text {-L- } \\
\text { rhamnopyranosyl- }(1 \rightarrow 2) \text { - }[\alpha \text {-L-rhamnopyranosyl- }(1 \rightarrow 3)]-\beta \text {-D-glucopyranoside }\end{array}$ & D. cambodiana & [45] \\
\hline 177 & $\begin{array}{c}\text { 1-O- } \alpha \text {-L-Rhamnopyranosyl- } 5 \alpha \text {-cholesta- } 1 \beta, 3 \beta, 16 \beta \text {-triol-7,23-dione } \\
\text { 16- } \beta \text {-D-glucopyranoside (concinnasteoside A) }\end{array}$ & D. concinna & {$[72]$} \\
\hline
\end{tabular}


Table 9. Cont.

\begin{tabular}{cccc}
\hline Number & Compound Name & Source & References \\
\hline $\mathbf{1 7 8}$ & $\beta$-Sitosterol 3-O- $\beta$-D-glucopyranoside (daucosterol) & D. draco & {$[29]$} \\
\hline $\mathbf{1 7 9}$ & $\beta$-Sitosterol 3-O-(6-O-palmitoyl)- $\beta$-D-glucopyranoside (sitoindoside I) & D. draco & {$[55]$} \\
\hline $\mathbf{1 8 0}$ & Stigmasterol 3-O- $\beta$-D-glucopyranoside & D. viridiflora & {$[50]$} \\
\hline $\mathbf{1 8 1}$ & $1 \beta$-Hydroxy-kryptogenin 1-O- $\alpha$-L-rhamnopyranosyl-(1-2)- $\alpha$-L-arabinopyranoside & S. cylindrica & {$[60]$} \\
\hline
\end{tabular}

Table 10. Saponin patterns of Dracaena and Sansevieria species.

\begin{tabular}{|c|c|c|}
\hline Species & Plant Parts Extracted & Saponins \\
\hline $\begin{array}{l}\text { Dracaena afromontana } \\
\text { Mildbr. }\end{array}$ & $\begin{array}{l}\text { methanolic extract of } \\
\text { the twigs }\end{array}$ & 139 \\
\hline $\begin{array}{l}\text { Dracaena angustifolia } \\
\text { Medik, (Roxb.) }\end{array}$ & $\begin{array}{l}\text { methanolic extract of } \\
\text { fresh stems; } \\
\text { roots and rhizomes }\end{array}$ & $\begin{array}{c}1,5,11,12,13,16,17,(23), 28,29.35,37,39,50,51,53,72,(75), 77,83,111,112 \\
113,127,140,143,147 a, 168,175\end{array}$ \\
\hline $\begin{array}{l}\text { Dracaena arborea } \\
\text { (Willd.) Link }\end{array}$ & $\begin{array}{l}\text { methanolic extract } \\
\text { of bark }\end{array}$ & $7,46,97,99,102$ \\
\hline $\begin{array}{l}\text { Dracaena cambodiana } \\
\text { Pierre ex Gagnep }\end{array}$ & $\begin{array}{l}\text { fresh stems; } \\
\text { dragon's blood }\end{array}$ & $\begin{array}{c}2,11,12,24,50,51,52,54,58,65,76,79,87,97,103,104,106,107,119,127,133 \\
164,165,168,173,176\end{array}$ \\
\hline $\begin{array}{l}\text { Dracaena cochinchinensis } \\
\text { (Lour.) S.C. Chen }\end{array}$ & $\begin{array}{l}\text { fresh stems } \\
\text { (dragon's blood) }\end{array}$ & $43,44,56,86,89,92,93,94,95,96,125,131,132,141,153,154,156,159,166,167,172,174$ \\
\hline Dracaena concinna Kunth & fresh stems & $28,36,82,85,88,117,136,137,138,144,160,163,177$ \\
\hline Dracaena draco L. & $\begin{array}{l}\text { stem bark; aerial parts; } \\
\text { leaves; roots }\end{array}$ & $\begin{array}{c}7,11,14,15,17,18,19,20,21,25,26,45,47,49,53,60,85,88,101,124,133,155 \\
157,178,179\end{array}$ \\
\hline $\begin{array}{c}\text { Dracaena fragrans (L.) } \\
\text { Ker Gawl. } \\
\text { (syn. D. deisteliana Engl.) }\end{array}$ & $\begin{array}{l}\text { methanolic extract of } \\
\text { stems; bark, } \\
\text { roots, leaves }\end{array}$ & $1,2,11,12,25,27,29,58,105$ \\
\hline Dracaena mannii Baker & fruit pulp; stem bark & $7,46,97$ \\
\hline Dracaena marginata Hort. & bark, roots & $6,28,30,32,40,41,77,81,90,128,129,150,158,162$ \\
\hline $\begin{array}{l}\text { Dracaena ombet Heuglin } \\
\text { ex Kotschy \& Peyr. }\end{array}$ & leaves & $22,73,74$ \\
\hline Dracaena surculosa Lindl. & $\begin{array}{l}\text { methanolic extract of } \\
\text { whole plant }\end{array}$ & $8,9,10,31,45,48,55,101,120,121,122,123,135$ \\
\hline $\begin{array}{l}\text { Dracaena thalioides Hort. } \\
\text { Makoy ex E. Morren }\end{array}$ & $\begin{array}{l}\text { leaves; fresh } \\
\text { underground parts }\end{array}$ & $1,4,11,32,34,46,58,72,77,80,97,98,100,108,111,112,114,115,118,130,147,149$ \\
\hline $\begin{array}{l}\text { Dracaena viridiflora Engl. } \\
\quad \& \text { K. Krause }\end{array}$ & leaves & $6,25,26,85,88,161,180$ \\
\hline $\begin{array}{l}\text { Sansevieria cylindrica } \\
\text { Bojer ex Hook. }\end{array}$ & $\begin{array}{l}\text { methanolic extract } \\
\text { of unflowering } \\
\text { aerial parts; leaves }\end{array}$ & $3,28,33,38,77,146,152,181$ \\
\hline $\begin{array}{l}\text { Sansevieria ehrenbergii } \\
\text { Schweinf. ex Baker }\end{array}$ & $\begin{array}{l}\mathrm{MeOH}-\mathrm{CH}_{2} \mathrm{Cl}_{2} \text { extract } \\
\text { of chipped plant }\end{array}$ & $25,42,57,84,88,91$ \\
\hline $\begin{array}{c}\text { Sansevieria trifasciata } \\
\text { Prain (syn. D. trifasciata } \\
\text { (Prain) Mabb) }\end{array}$ & $\begin{array}{l}\text { aerial parts; } \\
\text { methanol extract of the } \\
\text { whole plant }\end{array}$ & $\begin{array}{c}11,58,59,60,61,62,63,64,65,66,67,68,69,70,71,72,77,78,79,107,108,109 \\
110,111,114,116,126,134,142,145,148,151,168,169,170,171\end{array}$ \\
\hline
\end{tabular}



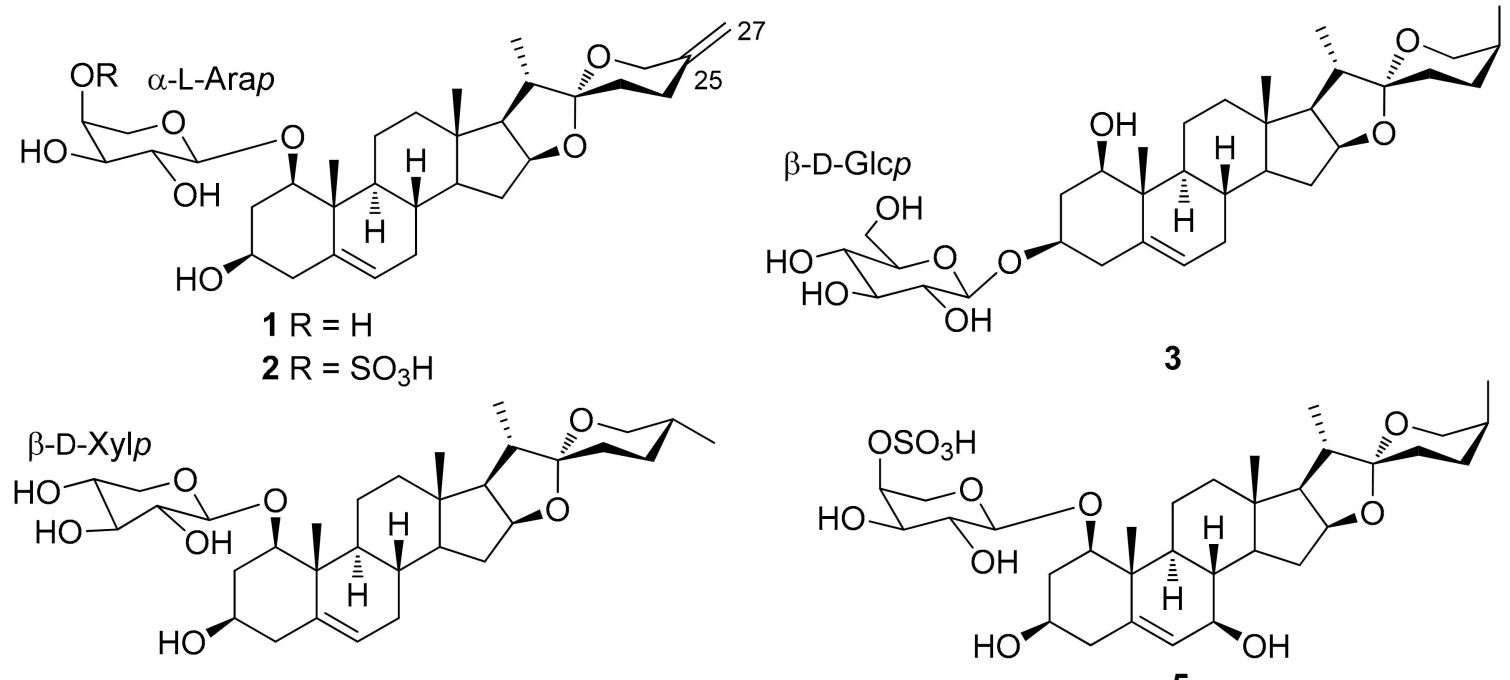

4
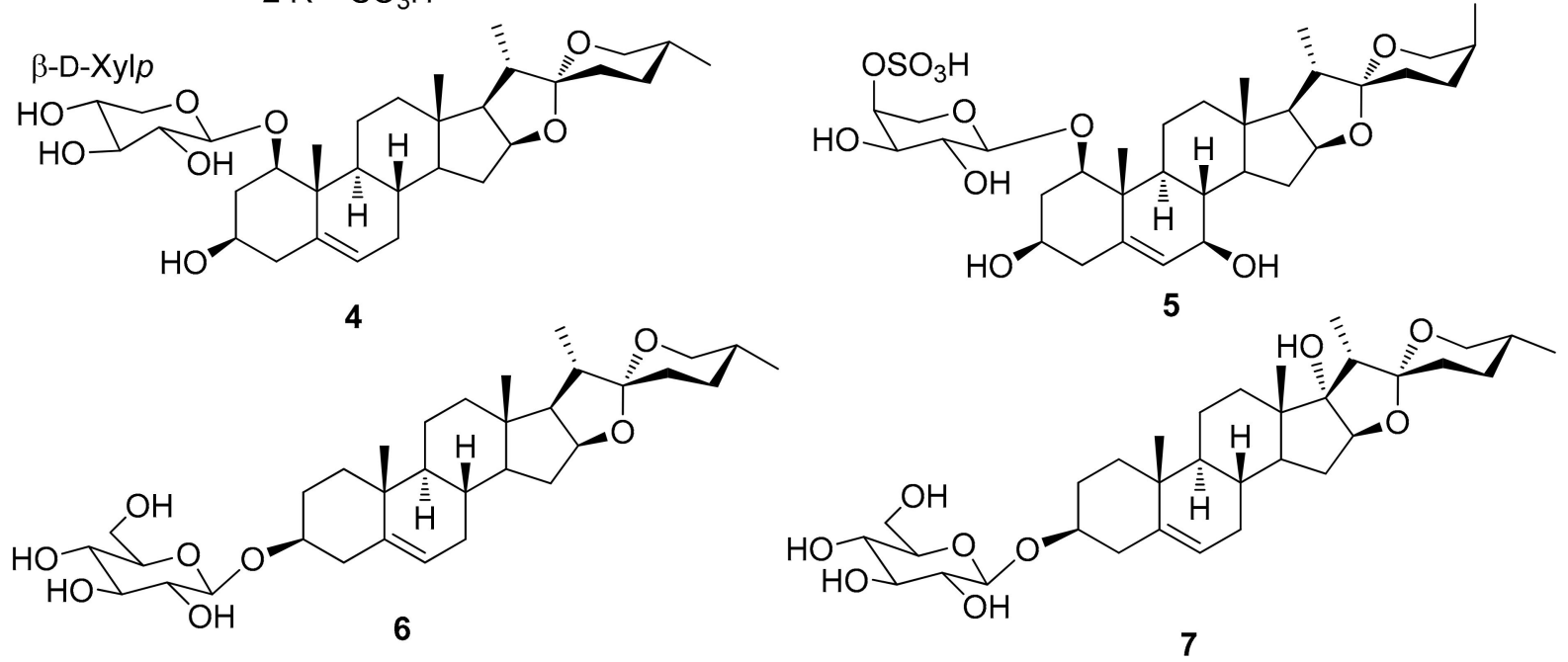

Figure 2. Chemical structures of spirostanol monoglycosides isolated from Dracaena and Sansevieria species.

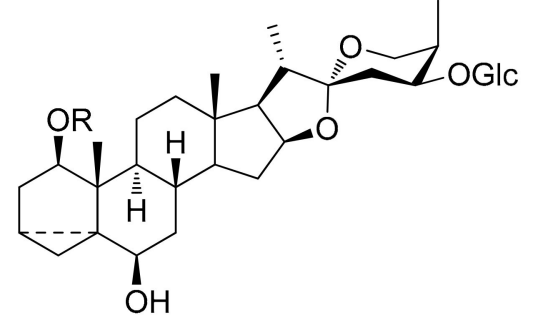

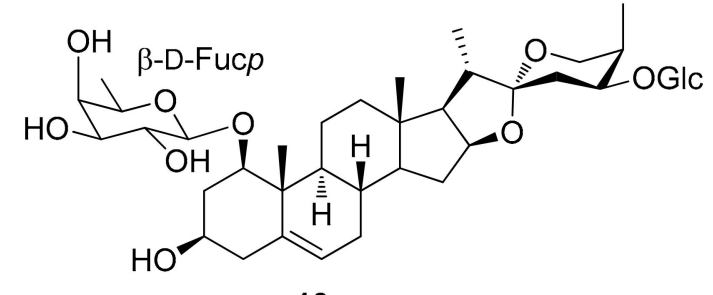

$8 \mathrm{R}=\beta-\mathrm{D}-\mathrm{Fucp}$

$9 \mathrm{R}=\beta-\mathrm{D}-\mathrm{Gl} c p c$

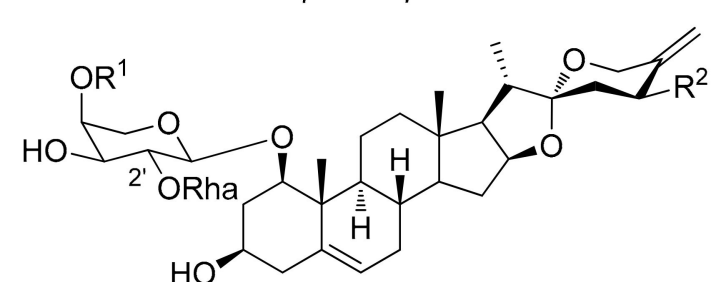

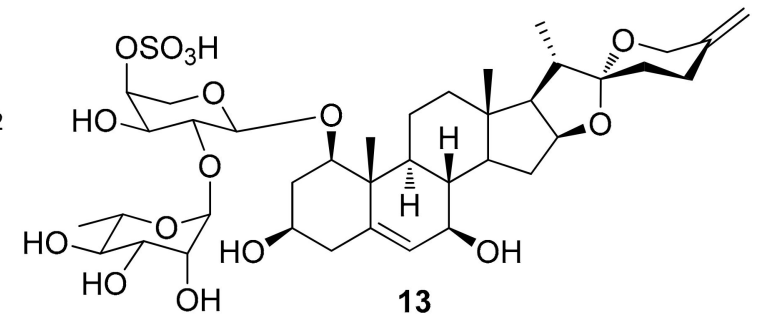

$$
\begin{aligned}
& 11 \mathrm{R}^{1}=\mathrm{R}^{2}=\mathrm{H} \\
& 12 \mathrm{R}^{1}=\mathrm{SO}_{3} \mathrm{HR}^{2}=\mathrm{H} \\
& 16 \mathrm{R}^{1}=\mathrm{SO}_{3} \mathrm{HR}^{2}=\mathrm{OH}
\end{aligned}
$$

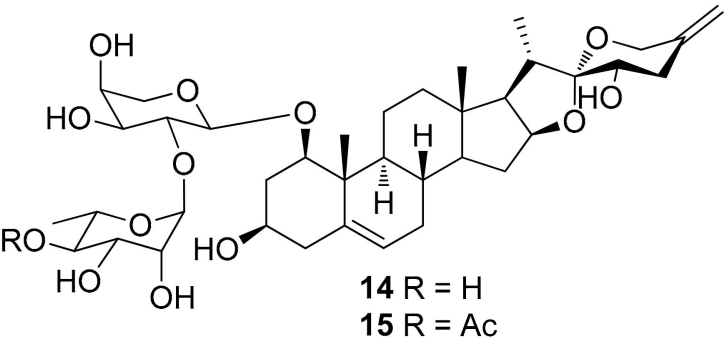

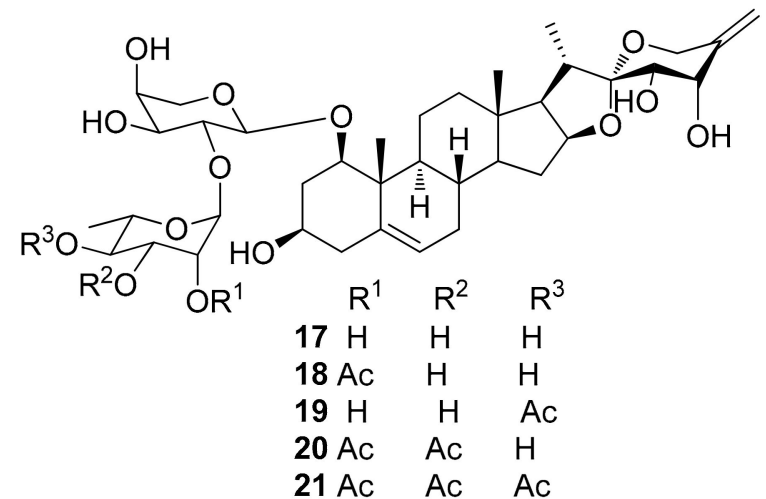

Figure 3. Cont. 

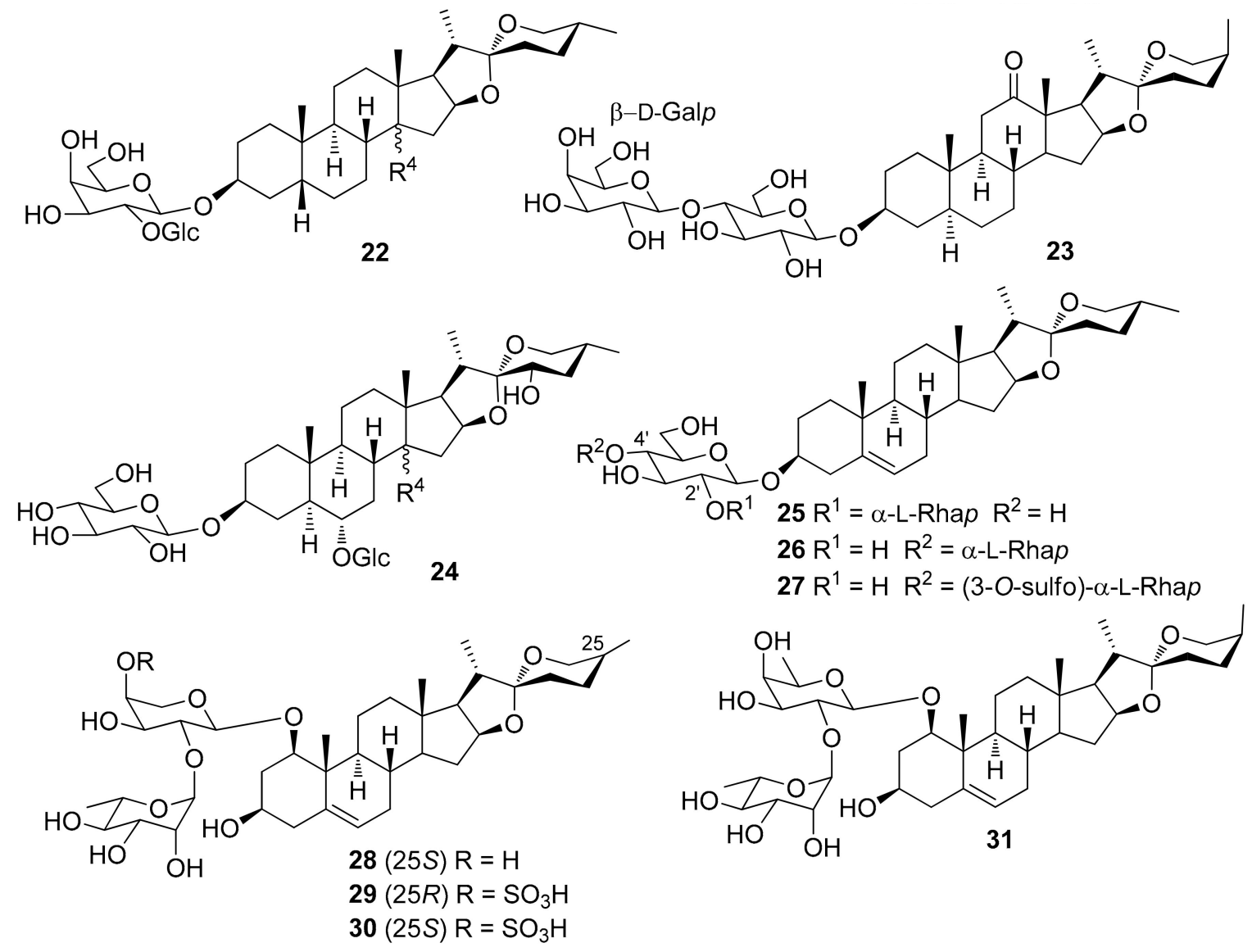

$29(25 R) \mathrm{R}=\mathrm{SO}_{3} \mathrm{H}$
$30(25 S) \mathrm{R}=\mathrm{SO}_{3} \mathrm{H}$
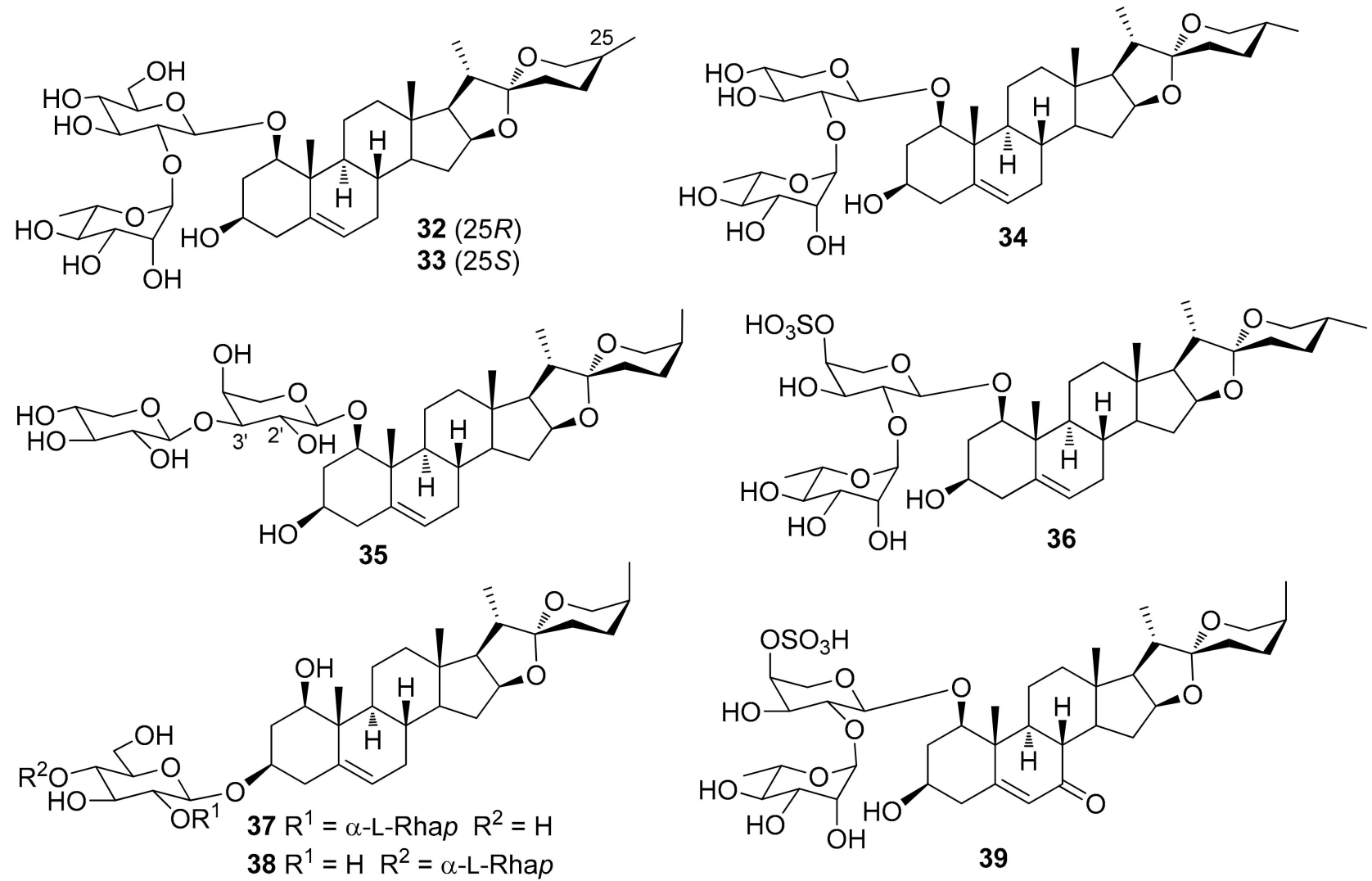

Figure 3. Cont. 


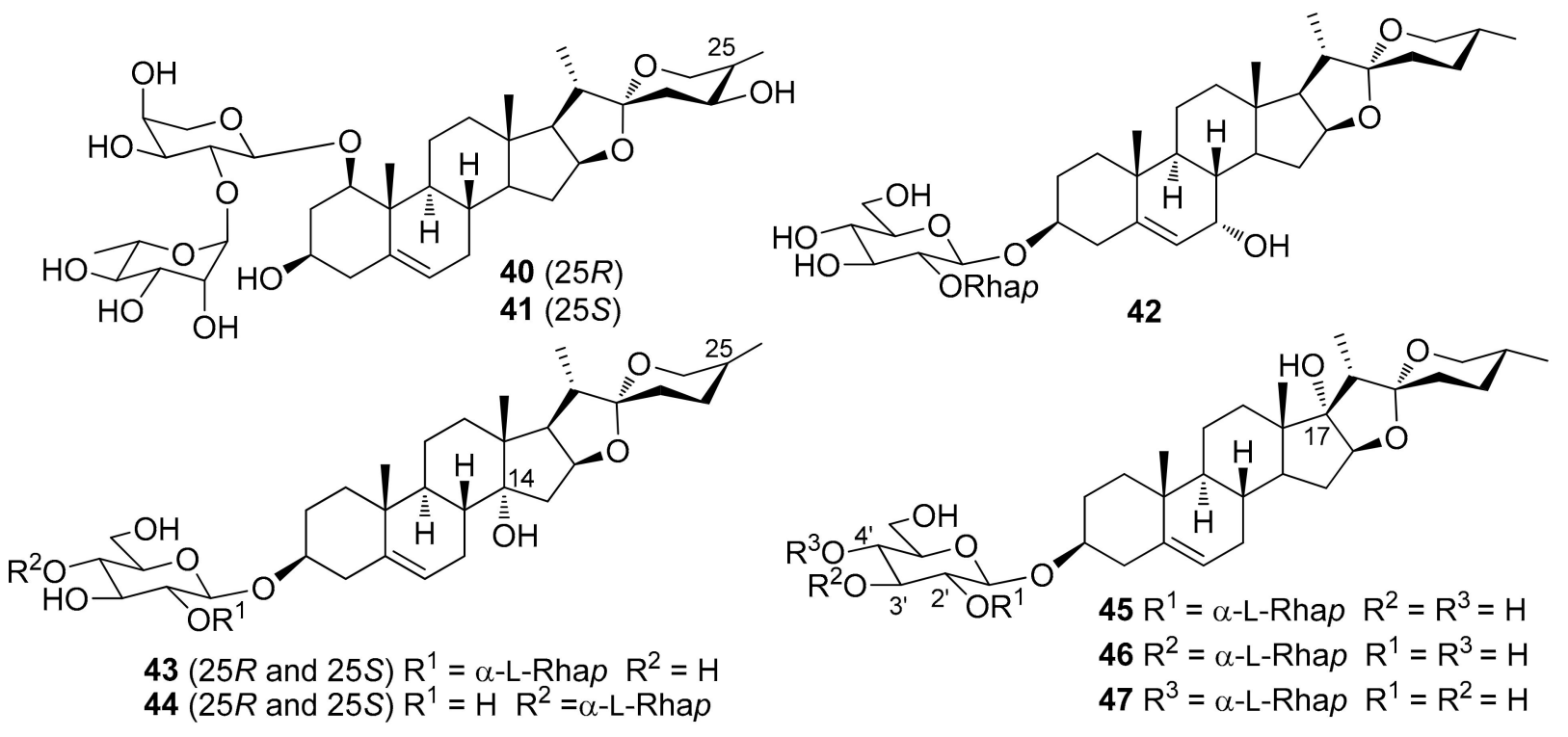

Figure 3. Chemical structures of spirostanol diglycosides isolated from Dracaena and Sansevieria species.

Table 10 shows the pattern of saponins in each Dracaena and Sansevieria species. It appears that each plant produces its own group of saponins. The richest species are D. angustifolia, D. cambodiana, D. cochinchinensis, D. draco, D. thalioides, and S. trifasciata. Saponins occurring in all species, that may be considered chemotaxonomic markers of the two genera, have not been isolated; saponins 11, 25, 28, 58, 77, 88, and 97 occur in more than three species.

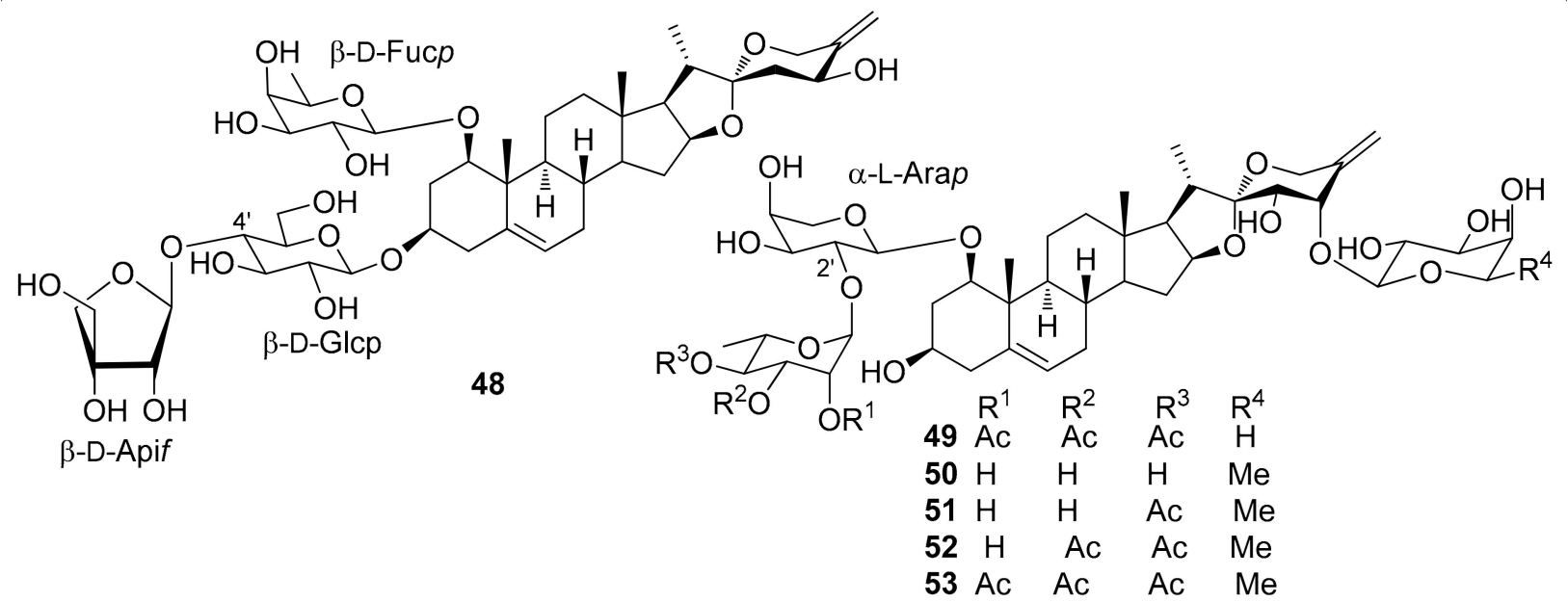

Figure 4. Cont. 


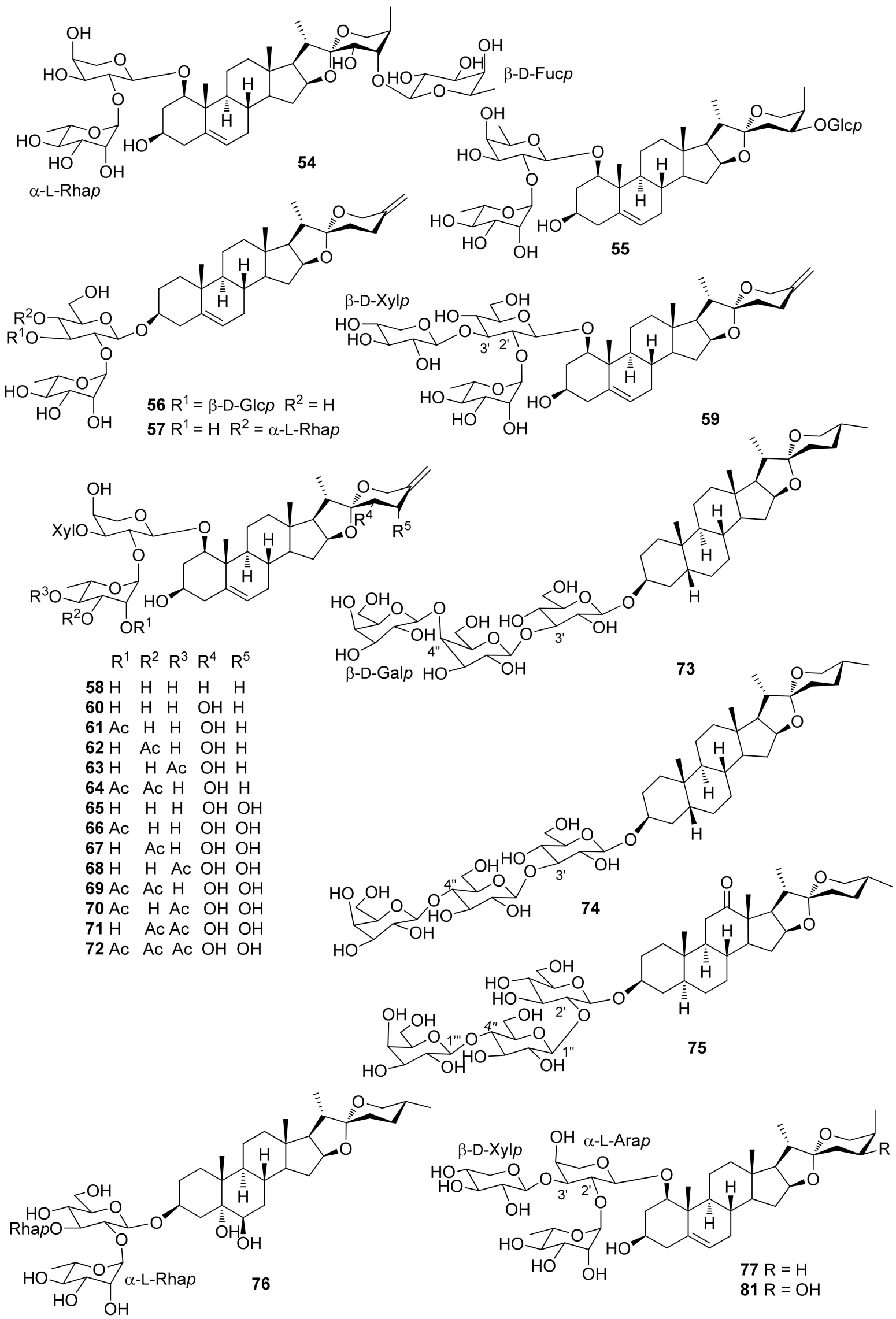

Figure 4. Cont. 


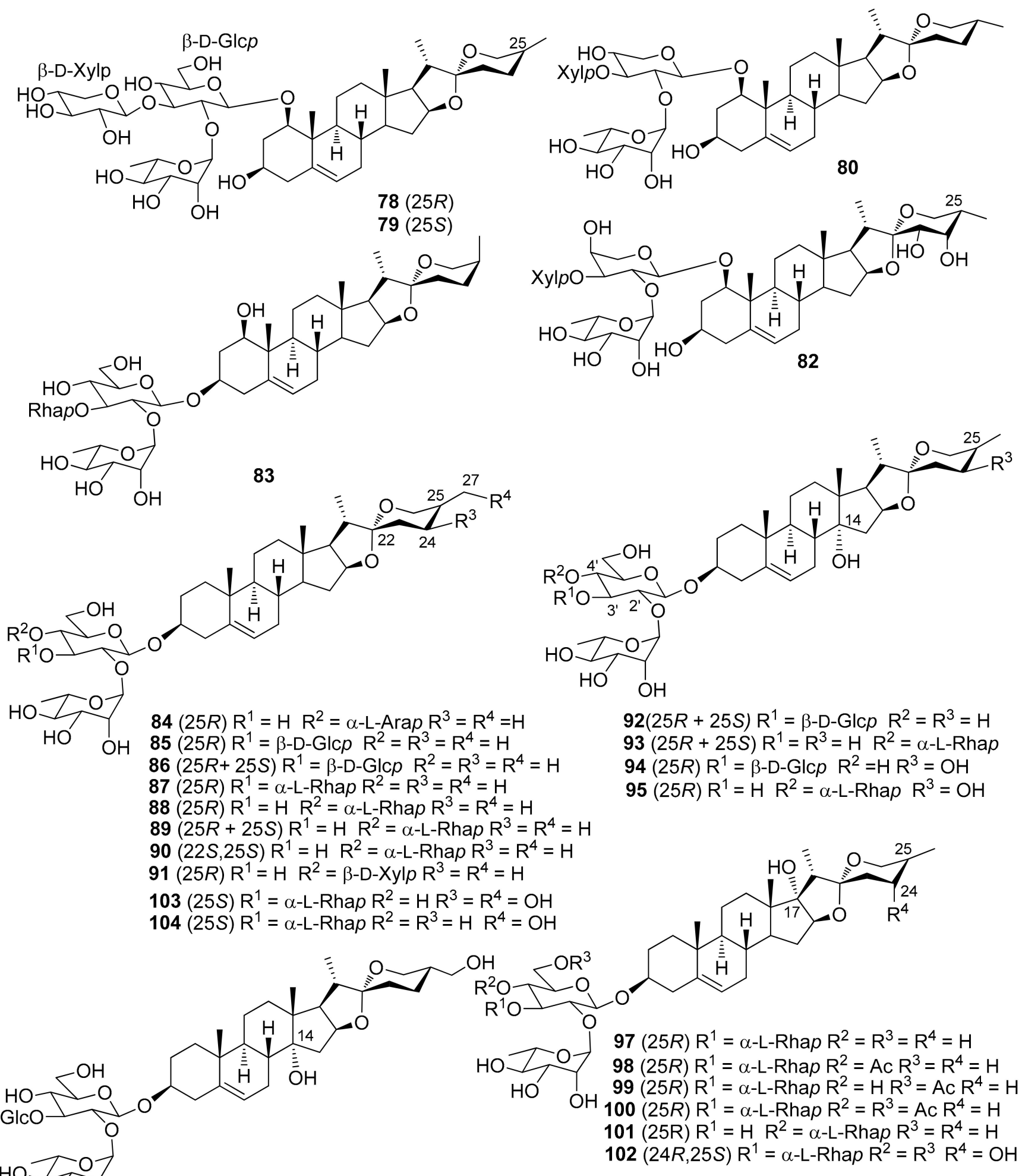

96

Figure 4. Chemical structures of spirostanol triglycosides isolated from Dracaena and Sansevieria species. 


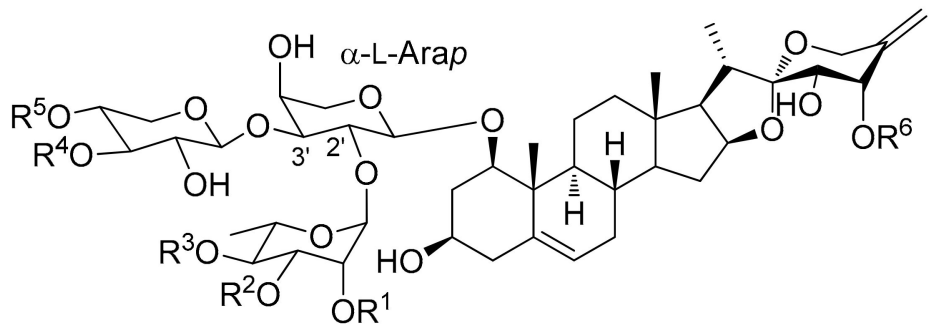

$\begin{array}{lllllll} & \mathrm{R}^{1} & \mathrm{R}^{2} & \mathrm{R}^{3} & \mathrm{R}^{4} & \mathrm{R}^{5} & \mathrm{R}^{6} \\ 106 & \mathrm{H} & \mathrm{H} & \mathrm{H} & \mathrm{H} & \mathrm{H} & \text { Fucp } \\ 107 & \mathrm{H} & \mathrm{H} & \mathrm{Ac} & \mathrm{H} & \mathrm{H} & \text { Fucp } \\ 108 & \mathrm{Ac} & \mathrm{Ac} & \mathrm{H} & \mathrm{H} & \mathrm{H} & \text { Fucp } \\ 109 & \mathrm{Ac} & \mathrm{H} & \mathrm{Ac} & \mathrm{H} & \mathrm{H} & \text { Fucp } \\ 110 & \mathrm{H} & \mathrm{Ac} & \mathrm{Ac} & \mathrm{H} & \mathrm{H} & \text { Fucp } \\ 111 & \mathrm{Ac} & \mathrm{Ac} & \mathrm{Ac} & \mathrm{H} & \mathrm{H} & \text { Fucp } \\ 112 & \mathrm{Ac} & \mathrm{Ac} & \mathrm{Ac} & \mathrm{Ac} & \mathrm{H} & \text { Fucp } \\ 113 & \mathrm{Ac} & \mathrm{Ac} & \mathrm{Ac} & \mathrm{H} & \mathrm{Ac} & \text { Fucp } \\ 114 & \mathrm{Ac} & \mathrm{Ac} & \mathrm{Ac} & \mathrm{H} & \mathrm{H} & \text { Glcp } \\ 115 & \mathrm{Ac} & \mathrm{Ac} & \mathrm{Ac} & \mathrm{Ac} & \mathrm{H} & \text { Glcp } \\ 116 & \mathrm{Ac} & \mathrm{Ac} & \mathrm{Ac} & \mathrm{H} & \mathrm{H} & \text { Rhap }\end{array}$
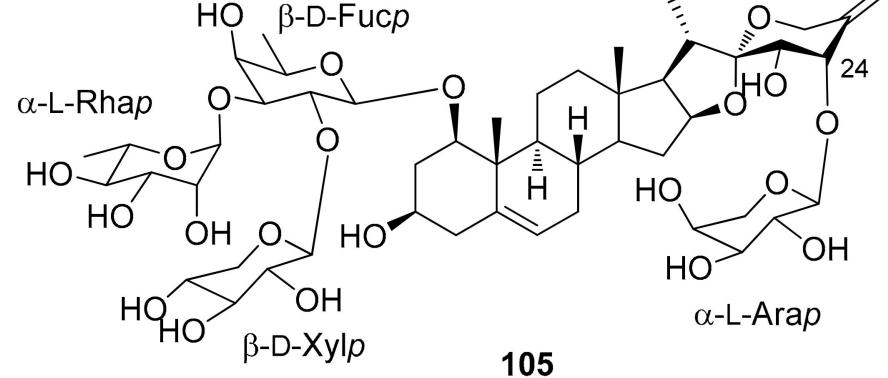

$\alpha$-L-Arap

105

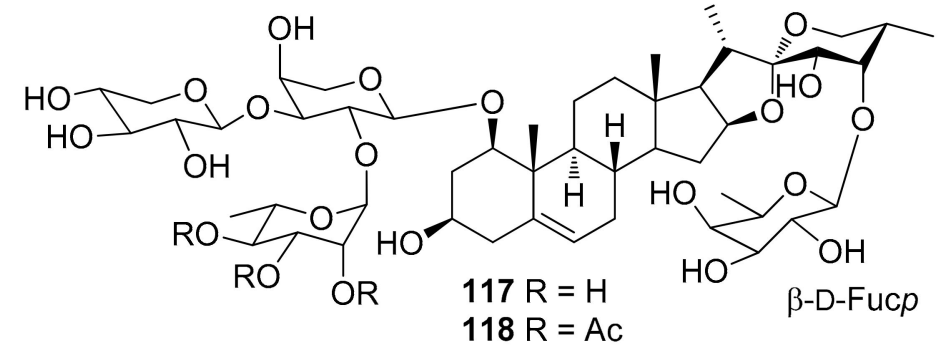

Figure 5. Chemical structures of spirostanol tetraglycosides isolated from Dracaena and Sansevieria species.

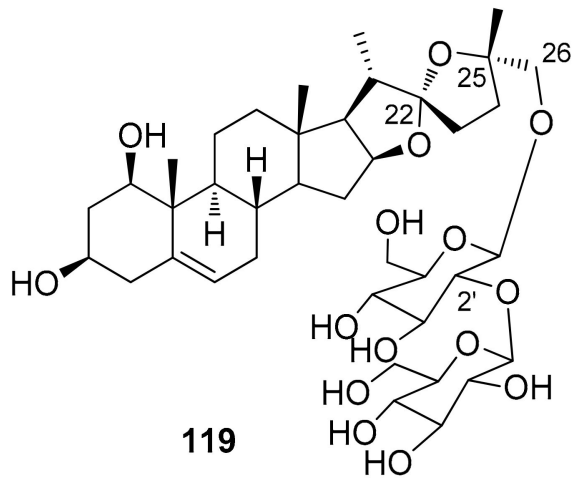

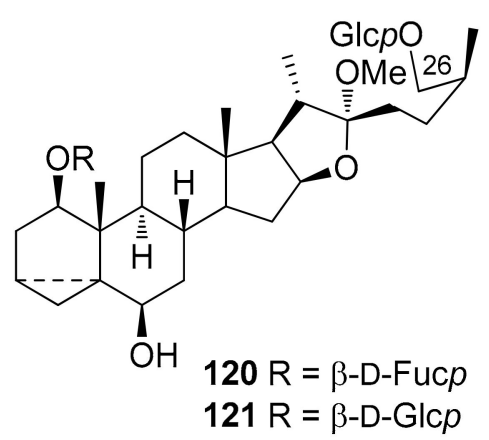

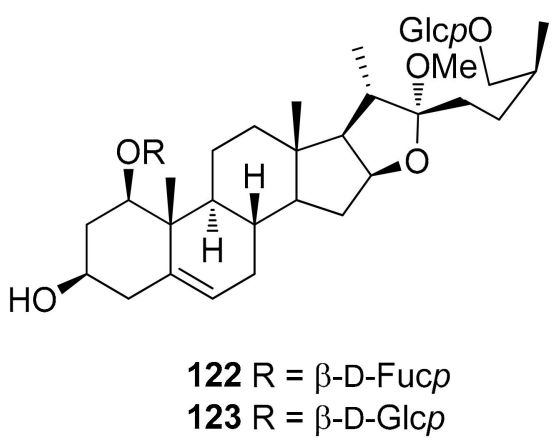

Figure 6. Chemical structures of furostanol diglycosides isolated from Dracaena species. 

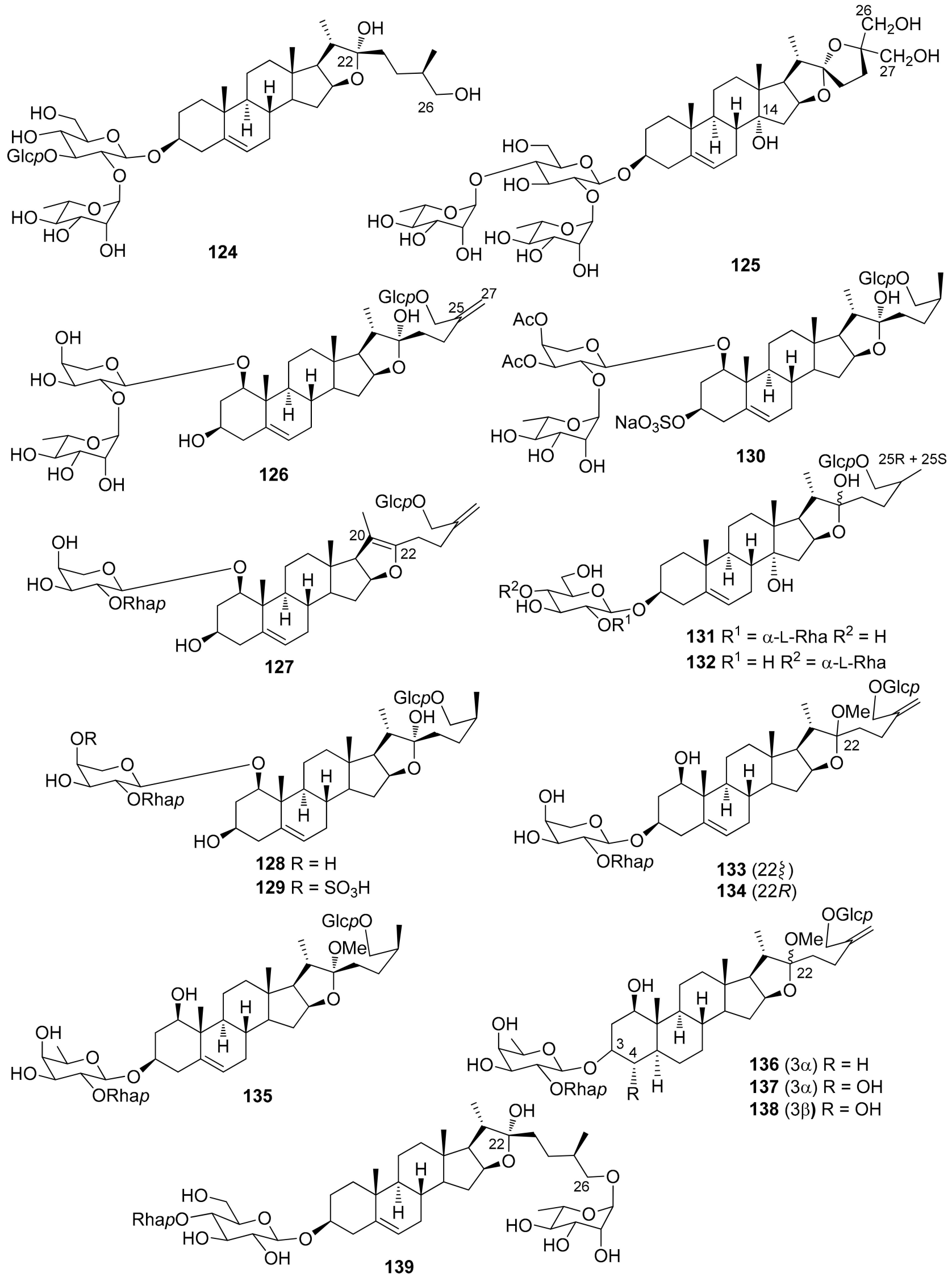

Figure 7. Chemical structures of furostanol triglycosides isolated from Dracaena and Sansevieria species. 


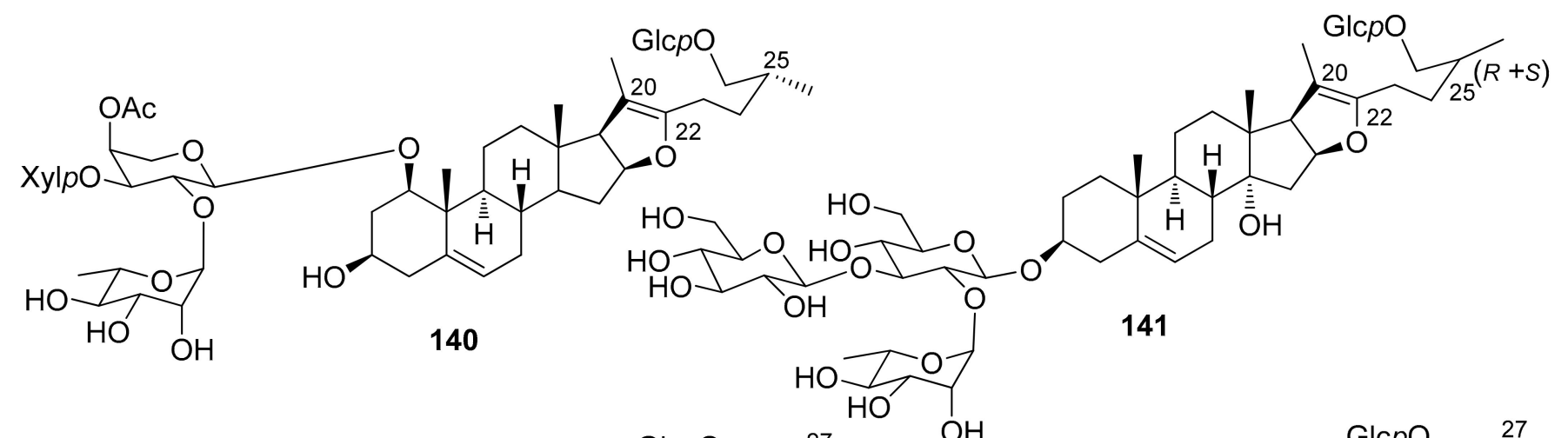

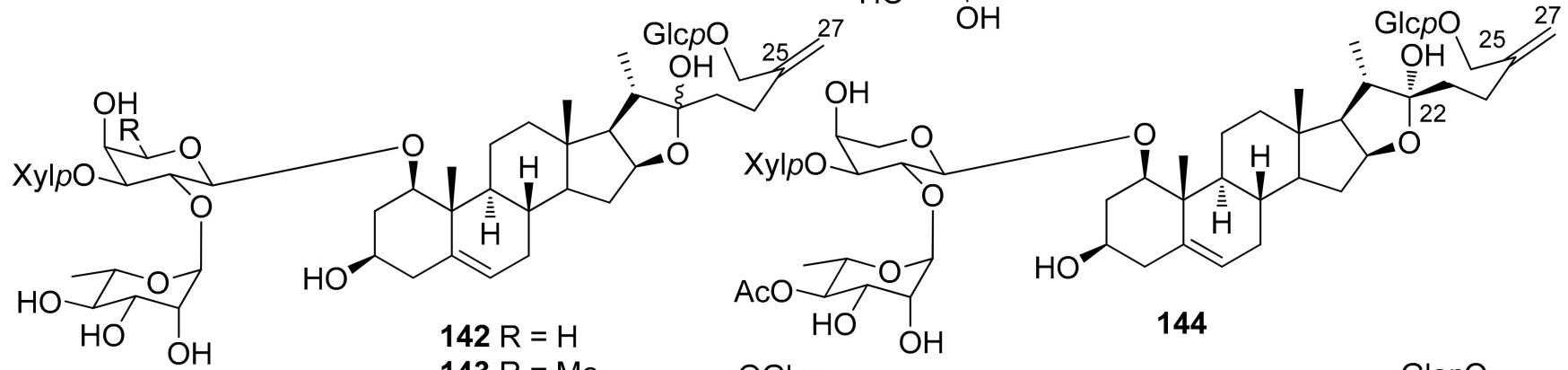

Figure 8. Cont. 

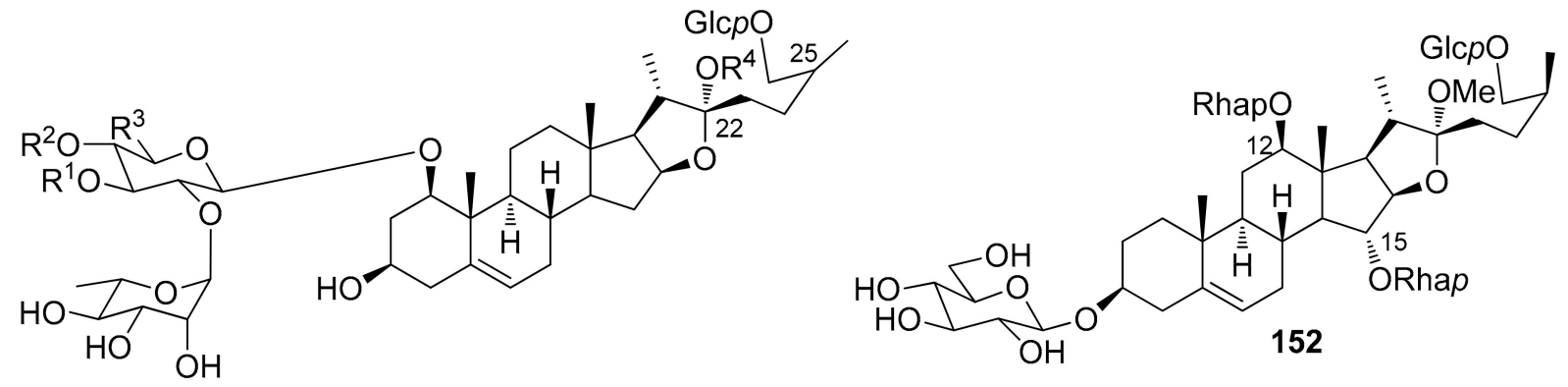

$$
\begin{aligned}
& 148 \text { (25S) } \mathrm{R}^{1}=\beta \text {-D-Xylp } \mathrm{R}^{2}=\mathrm{H} \mathrm{R}^{3}=\mathrm{CH}_{2} \mathrm{OH} \mathrm{R}^{4}=\mathrm{H} \\
& 149(25 R) R^{1}=\beta-D-X y l p R^{2}=R^{3}=R^{4}=H \\
& 150 \text { (25S) } \mathrm{R}^{1}=\mathrm{H} \mathrm{R}^{2}=\alpha-\mathrm{L}-\mathrm{Rhap} \mathrm{R}^{3}=\mathrm{CH}_{2} \mathrm{OH} \mathrm{R}^{4}=\mathrm{H} \\
& 151 \text { (25S) } \mathrm{R}^{1}=\beta \text {-D-Xylp R } \mathrm{R}^{2}=\mathrm{HR}^{3}=\mathrm{CH}_{2} \mathrm{OH} \mathrm{R}^{4}=\mathrm{Me}
\end{aligned}
$$
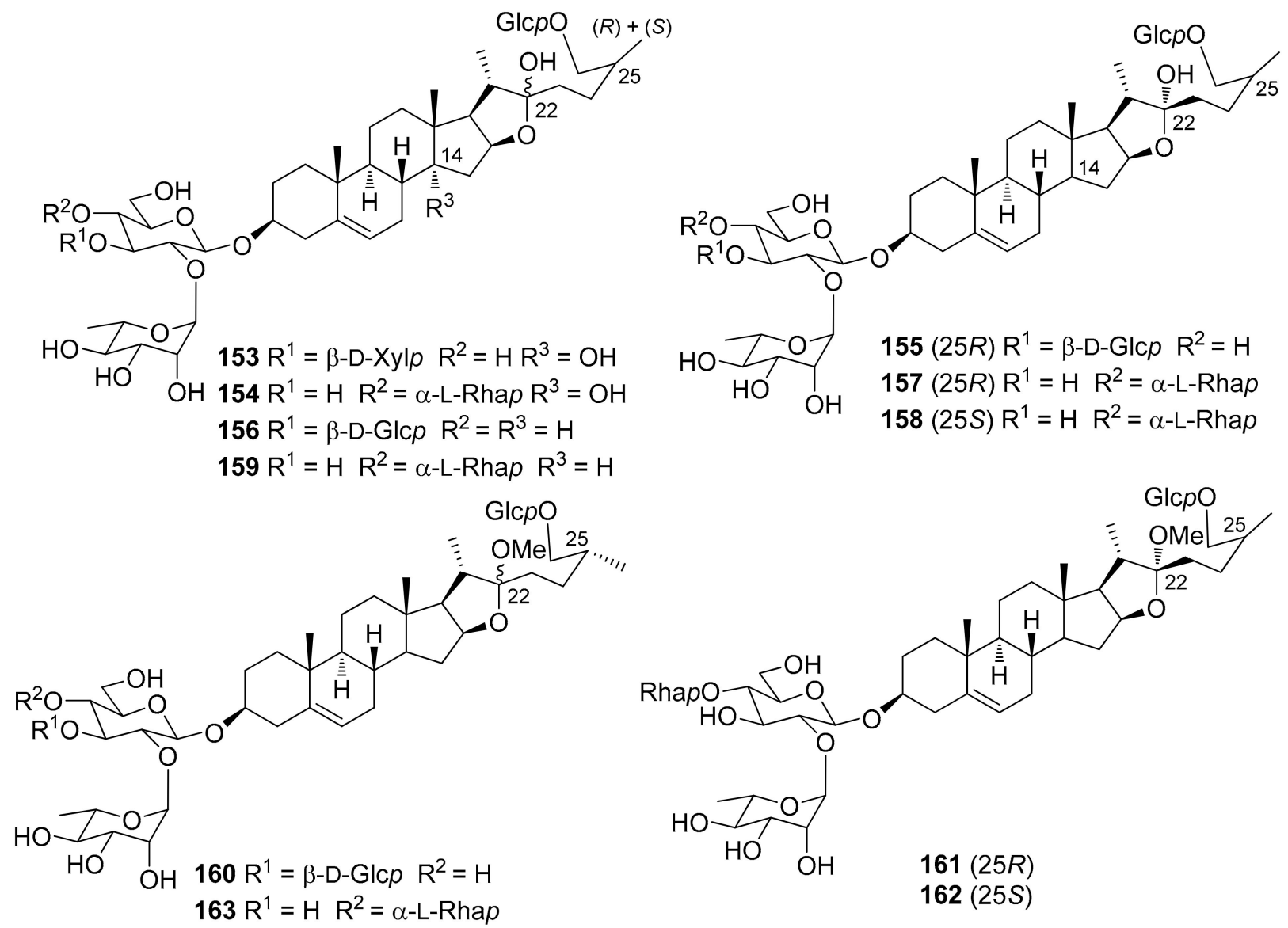

Figure 8. Chemical structures of furostanol tetraglycosides isolated from Dracaena and Sansevieria species. 


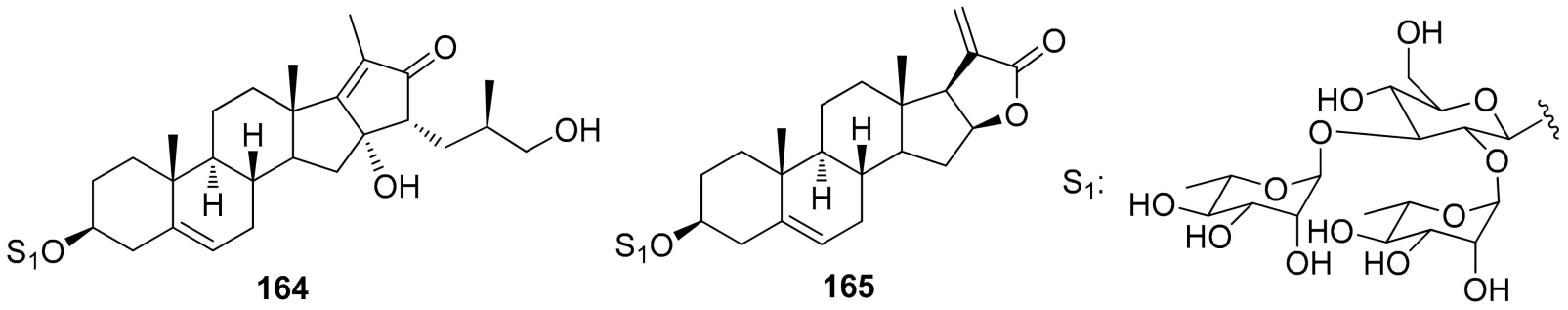

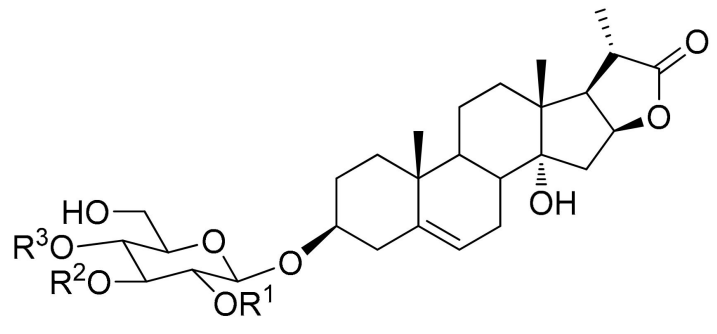

$$
\begin{array}{cccc} 
& R^{1} & R^{2} & R^{3} \\
166 & \text { Rha } & H & \text { Rha } \\
167 & \text { Rha } & \text { Glc } & H
\end{array}
$$

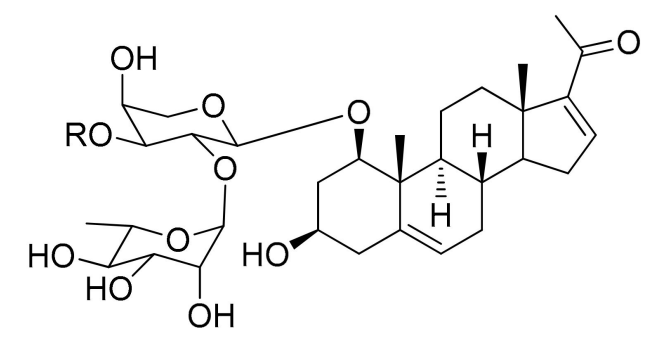

$168 \mathrm{R}=\mathrm{H}$

$169 \mathrm{R}=\beta-\mathrm{D}-\mathrm{Xylp}$

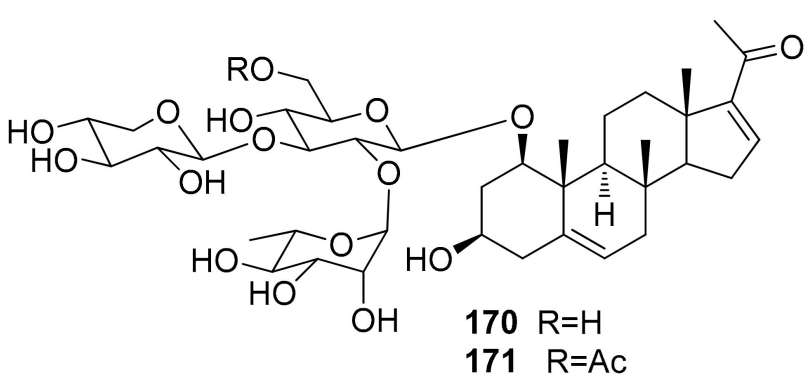

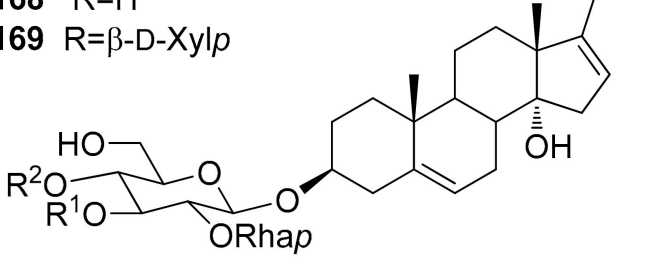

$$
\begin{array}{cc}
\mathrm{R}^{1} & \mathrm{R}^{2} \\
172 \beta-D-G l c p & \mathrm{H} \\
173 \alpha-\text {-Lhap } & \mathrm{H} \\
174 \quad \mathrm{H} \quad \alpha \text {-L-Rhap }
\end{array}
$$

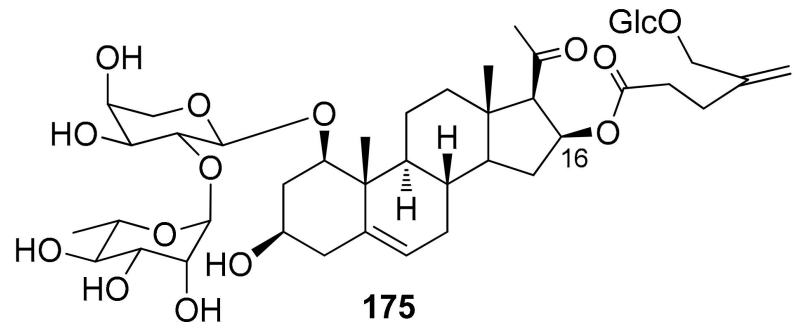

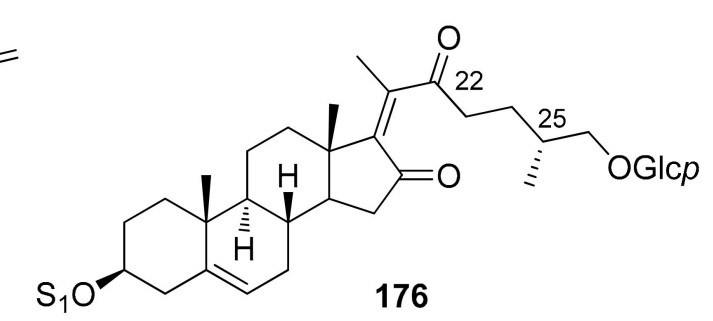

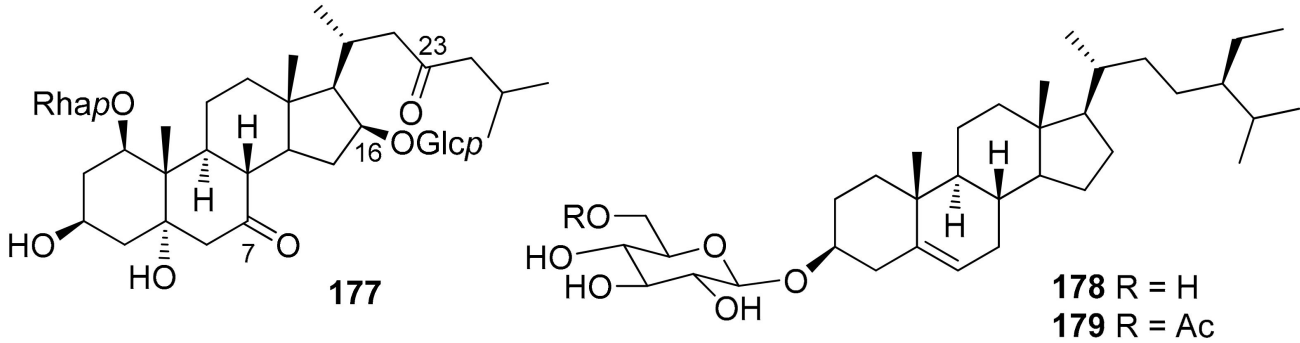

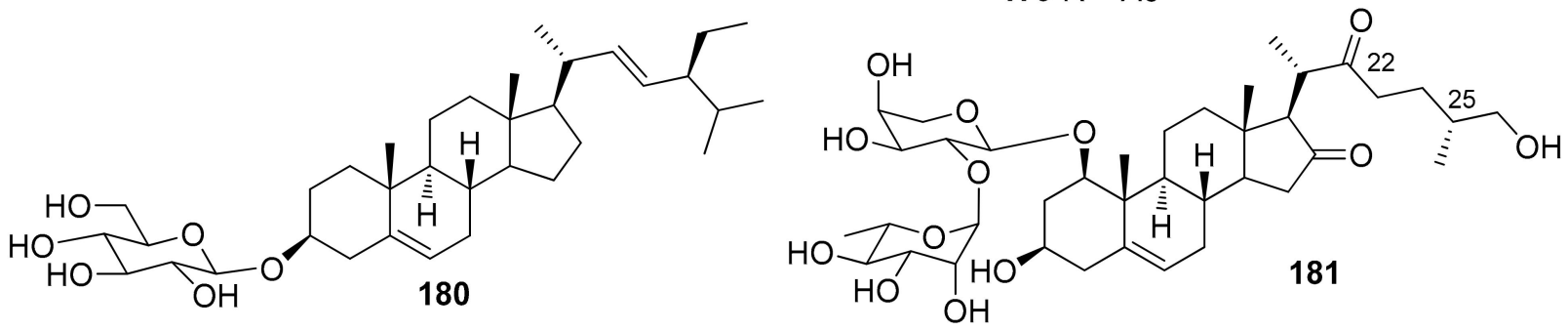

Figure 9. Chemical structures of miscellaneous steroidal saponins isolated from Dracaena and Sansevieria species. 


\section{Biological Activities}

The spectrum of biological effects tested for the saponins that are isolated from Dracaena and Sansevieria species is rather limited, and in vitro assays have been performed in most cases. In this review, we have reported the biological activities determined during the isolation of individual saponins from these two genera. The bioactivities of extracts or saponin-enriched fractions have not been discussed. Additional data, collected during the isolation of the same saponins from other genera, have not been reported and for them readers should consult the original literature.

\subsection{Hemolytic Properties}

Most saponins have powerful haemolytic activities because steroids have high affinities for cholesterol on erythrocyte membranes, thereby altering membrane permeability. Therefore, their toxicity is high when saponins are given intravenously to higher animals, while it is much less when administered orally. In this context, it is worthy of note that compound 152 showed no haemolytic effects in vitro [69]. This special behaviour was explained by assuming that the distribution of the sugar units around the aglycone moiety of the saponin considerably reduces its hydrophobicity, resulting in the loss of the amphipathic features. Moreover, saponin 152 inhibited the increase in capillary permeability caused by acetic acid, which is a typical model of first stage inflammatory reaction [69].

\subsection{Antiinflammatory Activity}

Pennogenin glycosides mannioside A (46) and floribundasaponin A (7) significantly inhibited carrageenan-induced paw edema in the rat, whereas spiroconazole A (97) was moderately active and aglycone pennogenin showed very weak activity [51]. The highest anti-inflammatory effects were observed one hour after carrageenan injection (Table 11), whereas the reference drug indomethacin reached a maximum of inhibition $(62.36 \%)$ at the 4th hour. These data underline the importance of the presence of a glycoside unit for the anti-inflammatory activity of pennogenin derivatives. The highest effects where observed when D-glucose was attached to $3-\mathrm{OH}$ of pennogenin and a rhamnosyl moiety was attached to $3^{\prime}-\mathrm{OH}[51]$.

Neutrophils play a significant role in the pathogenesis of several inflammatory diseases. The production of vast amounts of superoxide anion and elastase by activated neutrophils can cause tissue damage and contribute to the development of a wide spectrum of airway inflammatory diseases [58]. In this context, drangustosides A (83) and B (37) exhibited inhibitory activities from moderate to high against formyl-L-methionyl-Lleucyl-L-phenylalanine (fMLP)-induced superoxide anion production and elastase release in human neutrophils [58].

\subsection{Antimicrobial Activity}

A few compounds were very active against the pathogenic yeasts Cryptococcus neoformans and Candida albicans (Table 11), with most minimum inhibitory concentrations (MICs) in the $1-2 \mu \mathrm{g} / \mathrm{mL}$ range. Authors demonstrated that the antifungal activity of saponin 91 was fungicidal [57]. 
Table 11. Miscellaneous bioactivities of saponins isolated from Dracaena and Sansevieria species.

\begin{tabular}{|c|c|c|}
\hline Bioactivity & $\begin{array}{l}\text { Saponin } \\
\text { (Ref.) }\end{array}$ & Description \\
\hline Haemolytic effects & $152[69]$ & No haemolytic effects and inhibition of the capillary permeability activity \\
\hline \multirow{3}{*}{ Anti-inflammatory activity } & $7[51]$ & $\begin{array}{l}\text { Anti-inflammatory activity on carrageenan-induced paw edema } \\
\text { (maximum inhibitory activity of } 71.22 \% \text { ) }\end{array}$ \\
\hline & $46[51]$ & $\begin{array}{l}\text { Anti-inflammatory activity on carrageenan-induced paw edema } \\
\text { (maximum inhibitory activity of } 80.57 \% \text { ) }\end{array}$ \\
\hline & $97[51]$ & $\begin{array}{l}\text { Anti-inflammatory activity on carrageenan-induced paw edema } \\
\text { (maximum inhibitory activity of } 66.19 \% \text { ) }\end{array}$ \\
\hline \multirow{2}{*}{ Anti-neutrophilic inflammatory activity } & $37[58]$ & $\begin{array}{c}\text { Inhibitory activity against } \\
\text { formyl-L-methionyl-L-leucyl-L-phenylalanine-induced superoxide anion } \\
\text { generation }\left(\mathrm{IC}_{50}=18.55 \pm 0.23 \mu \mathrm{M}\right) \text { and elastase release by human } \\
\text { neutrophils }\left(\mathrm{IC}_{50}=1.74 \pm 0.25 \mu \mathrm{M}\right)\end{array}$ \\
\hline & $83[58]$ & $\begin{array}{c}\text { Inhibitory activity against } \\
\text { formyl-L-methionyl-L-leucyl-L-phenylalanine-induced superoxide anion } \\
\text { generation }\left(\mathrm{IC}_{50}=26.39 \pm 1.63 \mu \mathrm{M}\right) \text { and elastase release by human } \\
\text { neutrophils }\left(\mathrm{IC}_{50}=3.94 \pm 0.19 \mu \mathrm{M}\right)\end{array}$ \\
\hline \multirow{8}{*}{ Antimicrobial activity } & $25[57]$ & $\begin{array}{c}\text { Antimicrobial activities against Cryptococcus neoformans }(\mathrm{MIC}=1 \mu \mathrm{g} / \mathrm{mL}) \\
\text { and Candida albicans }(\mathrm{MIC}=2 \mu \mathrm{g} / \mathrm{mL})\end{array}$ \\
\hline & $28[48]$ & Antifungal activity against Cryptococcus neoformans $\left(\mathrm{IC}_{50}=20.0 \mu \mathrm{g} / \mathrm{mL}\right)$ \\
\hline & $32[62]$ & Antibacterial activity against Staphylococcus aureus \\
\hline & $35[48]$ & Antifungal activity against Cryptococcus neoformans $\left(\mathrm{IC}_{50}=9.5 \mu \mathrm{g} / \mathrm{mL}\right)$ \\
\hline & $57[57]$ & $\begin{array}{l}\text { Antimicrobial activities against Cryptococcus neoformans } \\
(\mathrm{MIC}=1-2 \mu \mathrm{g} / \mathrm{mL} \text { ) and Candida albicans (MIC }=2 \mu \mathrm{g} / \mathrm{mL})\end{array}$ \\
\hline & $84[57]$ & $\begin{array}{l}\text { Antimicrobial activities against Cryptococcus neoformans } \\
\text { (MIC }=1-2 \mu \mathrm{g} / \mathrm{mL})\end{array}$ \\
\hline & 88 [57] & $\begin{array}{l}\text { Antimicrobial activities against Cryptococcus neoformans } \\
(\mathrm{MIC}=1-2 \mu \mathrm{g} / \mathrm{mL} \text { ) and Candida albicans (MIC }=2 \mu \mathrm{g} / \mathrm{mL})\end{array}$ \\
\hline & $91[57]$ & $\begin{array}{c}\text { Antimicrobial activities against Cryptococcus neoformans }(\mathrm{MIC}=2 \mu \mathrm{g} / \mathrm{mL} \text { ) } \\
\text { and Candida albicans (MIC }=4-8 \mu \mathrm{g} / \mathrm{mL})\end{array}$ \\
\hline Molluscicidal activity & $97[66]$ & $\begin{array}{l}\text { At the concentration of } 5-6 \mathrm{ppm} \text {, spiroconazole A caused } 100 \% \text { mortality } \\
\text { of the snails Bulinus globosus, } B \text {. forskalii, Biomphalaria pfeifferi, B. glabrata, } \\
\text { and Lymnaea natalensis within } 24 \text { h. Other two related (unidentified) } \\
\text { saponins were lethal with } \mathrm{LC}_{50} \text { values in the range of } 10-25 \mathrm{ppm}\end{array}$ \\
\hline
\end{tabular}

\subsection{Molluscicidal Activity}

Some snails, especially of the genera Bulinus, Biomphalaria or Oncomelania are implicated in the transmission of schistosomiasis (bilharzia). The infection is caused by cercariae, liberated from the intermediate host snail, that penetrate the skin and mainly affect the genitourinary and gastro-intestinal systems. Three spirostanol saponins, pennogenin glycosides, isolated from the methanolic extract of the fruit pulp of Dracaena mannii, exhibited significant molluscicidal activity when tested against 5 snail vectors [66]. The highest activity was shown by spiroconazole A (97) whose LC $_{50}$ values were 2.95 ppm (Bulinus globosus), 4.36 ppm (B. forskalii) and 3.63 ppm (Lymnaea natalensis), respectively [66]. The aglycones showed no molluscicidal activity, indicating that the sugar moiety is an essential part of the molecules to retain the activity.

\subsection{Cell Antiproliferative/Cytotoxic Activity}

A relatively vast number of saponins, isolated from Dracaena and Sansevieria species, have been tested for their antiproliferative/cytotoxic activities in vitro (Table 12). However, the data are hardly comparable due to the fact that different cell lines and procedures were 
often used in different laboratories. The choice of cell lines (usually from one to five) in most reports, cited in this review, seemed to be random. It is, thus, highly recommendable that a standardized procedure is followed in future investigations. Moreover, antiproliferative activity and cytotoxicity have often been considered as synonyms and the terms, antiproliferation (cytostatic effects) and cytotoxicity (killing effects), have been used interchangeably. Most assays have been performed with a colorimetric method (MTT, XTT), that is based on the reduction of tetrazolium salts in living cells by mitochondrial dehydrogenases. This assay allows, indeed, the estimation of the activity of metabolically active cells (viable cells), while offering little insight into the mechanisms concerning antiproliferative versus cytotoxic effects. The sulforhodamine B (SRB) assay has also been used to determine cell growth inhibition [49]. DNA damage and the consequent induction of apoptosis are principal cytotoxic mechanisms of several anticancer agents. Therefore, in certain cytotoxicity assays DNA fragmentation, condensation of chromatin and other morphological changes are visualized and quantified by fluorescence microscopy. Immunoblot (Western blot) analysis is also performed to determine cleavage of poly(ADP-ribose) polymerase-1 (PARP), which it is a key enzyme in the apoptosis process. Moreover, the cytotoxic effects of some steroidal saponins may result from their nonspecific detergent effects on membrane architecture [57], even if there is no clear correlation between the haemolytic activity and cytotoxicity of steroidal saponins [73].

We have maintained the distinction between cytostatic and cytotoxic activities. Table 12 show the cell growth inhibition activities of saponins as $\mathrm{IC}_{50}$ values, i.e., the concentration at which $50 \%$ inhibition of cell growth was observed. For homogeneity, the values expressed in literature in $\mu \mathrm{g} / \mathrm{mL}$ have been converted to $\mu \mathrm{M}$. Human tumour cell lines were used where not otherwise indicated. $\mathrm{IC}_{50}$ values up to $30 \mu \mathrm{M}$ have been included in the table, even if several authors consider the activity of a compound worthy of note only if the $\mathrm{IC}_{50}$ is $<10 \mu \mathrm{M}$. Indeed, a great number of saponins from Dracaena and Sansevieria exhibited $\mathrm{IC}_{50}$ values in the range of $1-10 \mu \mathrm{M}$, and some in the submicromolar range. In addition, several saponins exhibited higher activity than the reference compound (e.g., etoposide, paclitaxel, doxorubicin, 5-fluorouracil) on certain cell lines.

A rather comprehensive review on saponin cytotoxicity was published a few years ago [73]. The structure-activity relationships of saponins are generally difficult to decipher, and only a fairly limited number of comparative studies have been focused on the establishment of structure-activity relationships, relevant to activity [73]. From the data reported in Table 12, and others in the literature, it appears that the a glycoside unit is essential for cytostatic/cytotoxic activities of steroidal saponins. Moreover, the activity is sensitive to the monosaccharides constituting the sugar moieties, and their number and sequences, the glycosylation site, as well as the structures of the aglycones. For example, the $25 S$ configuration appeared to be critical for activity against leukemia cells but not against solid tumors [73]. Furthermore, the presence of oxygenated functional groups on some positions of the aglycones, e.g., at C-7, seems to reduce the activity. The 1-O glycosylated saponins 111, 112 and 118 were the most active among a number of saponins tested against promyelocytic leukemia HL-60 cells; they showed $\mathrm{IC}_{50}$ values comparable or even higher than the well-known chemotherapeutic agents cisplatin and etoposide (Table 12). The authors suggested that the triacetylated $\alpha$-L-rhamnopyranosyl moiety at C- ${ }^{\prime}$ and the $\beta$-Dfucopyranosyl group attached to C-24 of the aglycone play important roles in imparting potent antiproliferative activity against HL-60 cells [32]. However, dioscin (88) bearing a non-acylated Rhap-( $1 \rightarrow 2)-[$ Rhap- $(1 \rightarrow 4)]$-Glcp moiety at C-3 of the aglycone, was also quite active against HL-60 cells [28], while fully acetylated dioscin was inactive against several cell lines, contrary to dioscin [49]. In the dioscin series, comparison of the activity of $2^{\prime}, 4^{\prime}$-di-O-rhamnopyranosyl-3-O-glucopyranoside 88 with those of monorhamnosyl derivatives 25 and 26 (Table 12), and inactive monoglycosylated trillin 6 [50], clearly shows that the antiproliferative activity is greatly increased in the dirhamnosyl derivative, while the presence of a rhamnosyl unit at C-2' is critical to the activity. Substitution of the $4^{\prime}-$ rhamnosyl unit in dioscin with an arabinopyranosyl or a xylopyranosyl moiety, slightly 
modifies the activity (cfr. 88 with 84 and 91, Table 12). Icodeside (49) and draconin A (21), exhibited similar antiproliferative activity towards HL-60 cells (IC ${ }_{50}$ ca. 9) [28,29], which indicates that the additional arabinopyranosyl moiety in 49 , compared to draconin A, does not affect the activity significantly. Considering the antiproliferative activity of the saponins isolated from the roots and rhizomes of D. angustifolia [26], the authors concluded that the spirostanol saponins possess more potent antiproliferative activity than their furostanol analogues. In contrast, furostanol icogenin (88) is even slightly more active than the correspondin spirostanol gracillin (85) against HL-60 cells [55]. A 24-O-fucopyranosyl substituent, together with a triacetylated $\alpha$-L-rhamnopyranosyl moiety and a xylopranosyl unit attached to the $2^{\prime}-O$ and $3^{\prime}-O$, respectively, of an arabinopyranosyl substituent at 1-O seem to be important for the activity against HT-1080 fibrosarcoma cells (Table 12).

In addition to antiproliferative activities, saponins 20, 21, 85, 112, 124 also exhibited significantly cytotoxic properties $[28,32,55]$ and some mechanisms of action have been clarified. For example, the potent anticancer agent gracillin (85) showed significant inhibitory effect on mitochondrial complex II-mediated energy production in non-small cell lung cancer, and it inhibited glycolysis and oxidative phosphorylation-mediated bioenergetics [74].

Table 12. Antiproliferative activities of saponins isolated from Dracaena and Sansevieria species.

\begin{tabular}{|c|c|}
\hline $\begin{array}{l}\text { Drug } \\
\text { (Ref.) }\end{array}$ & $\begin{array}{r}\text { Cell Line } \\
\mathrm{IC}_{50}(\mu \mathrm{M}) \\
\end{array}$ \\
\hline & $\begin{array}{l}\text { Promye- } \\
\text { locytic } \\
\text { leukemia } \\
\text { HL-60 }\end{array}$ \\
\hline $\begin{array}{c}\text { cisplatin } \\
\text { [32] }\end{array}$ & $1.40 \pm 0.08$ \\
\hline $\begin{array}{c}\text { etoposide } \\
\text { [32] }\end{array}$ & $0.38 \pm 0.06$ \\
\hline $1[32]$ & $9.34 \pm 2.93$ \\
\hline $4[32]$ & $7.38 \pm 0.78$ \\
\hline 11 [32] & $7.85 \pm 0.43$ \\
\hline 32 [32] & $17.3 \pm 2.99$ \\
\hline 34 [32] & $12.3 \pm 2.56$ \\
\hline $46[32]$ & 20 \\
\hline 59 [32] & $9.45 \pm 2.22$ \\
\hline 73 [32] & $4.45 \pm 0.39$ \\
\hline 80 [32] & $11.3 \pm 1.21$ \\
\hline 97 [32] & $6.36 \pm 0.14$ \\
\hline 98 [32] & $7.64 \pm 0.59$ \\
\hline $100[32]$ & $>20$ \\
\hline 108 [32] & $6.00 \pm 1.22$ \\
\hline $111[32]$ & $0.47 \pm 0.04$ \\
\hline 112 [32] & $0.38 \pm 0.04$ \\
\hline 114 [32] & $2.73 \pm 0.42$ \\
\hline 115 [32] & $1.66 \pm 0.20$ \\
\hline 118 [32] & $0.74 \pm 0.05$ \\
\hline $21[28]$ & $9.7 \pm 2.7$ \\
\hline $85[28]$ & $3.7 \pm 0$ \\
\hline 85 [55] & $4.0 \pm 0.4$ \\
\hline $88[28]$ & $2.0 \pm 0.9$ \\
\hline 88 [55] & $2.3 \pm 0.8$ \\
\hline
\end{tabular}


Table 12. Cont.

\begin{tabular}{|c|c|c|c|c|c|c|c|c|c|}
\hline $\begin{array}{l}\text { Drug } \\
\text { (Ref.) }\end{array}$ & & & & & $\begin{array}{l}\text { Cell Line } \\
\mathrm{IC}_{50}(\mu \mathrm{M})\end{array}$ & & & & \\
\hline 155 [28] & $7.2 \pm 2.3$ & & & & & & & & \\
\hline 157 [28] & $7.3 \pm 3.7$ & & & & & & & & \\
\hline 49 [29] & $9 \pm 4$ & & & & & & & & \\
\hline $\begin{array}{c}\text { etoposide } \\
\text { [29] }\end{array}$ & 0.2 & & & & & & & & \\
\hline 25 [53] & 1.8 & & & & & & & & \\
\hline 53 [53] & 2.5 & & & & & & & & \\
\hline $\begin{array}{c}\text { etoposide } \\
\text { [53] }\end{array}$ & 0.5 & & & & & & & & \\
\hline 124 [55] & $2.6 \pm 0.9$ & $\begin{array}{c}\text { Epi- } \\
\text { dermoid } \\
\text { carcinoma } \\
\text { A-431 }\end{array}$ & $\begin{array}{l}\text { HeLa } \\
\text { (derived } \\
\text { from } \\
\text { cervical } \\
\text { cancer } \\
\text { cells) } \\
\end{array}$ & $\begin{array}{l}\text { Colo rectal } \\
\text { cancer } \\
\text { HCT116 }\end{array}$ & $\begin{array}{c}\text { Hepato } \\
\text { cyte } \\
\text { carcinoma } \\
\text { HepG2 }\end{array}$ & $\begin{array}{c}\text { Breast carci } \\
\text { noma } \\
\text { MCF7 }\end{array}$ & $\begin{array}{c}\text { Myelo } \\
\text { genous } \\
\text { leuke mia } \\
\text { K-562 }\end{array}$ & $\begin{array}{c}\text { Hepatoma } \\
\text { BEL-7402 }\end{array}$ & $\begin{array}{c}\text { Gastric } \\
\text { cancer } \\
\text { SGC-7901 }\end{array}$ \\
\hline 49 [29] & & 16.1 & & & & & & & \\
\hline 109 [33] & & & 26.5 & & & & & & \\
\hline 110 [33] & & & 26.5 & & & & & & \\
\hline 33 [47] & & & & & & $29.6 \pm 1.4$ & & & \\
\hline 38 [47] & & & & $16.9 \pm 1.4$ & $15.5 \pm 2.8$ & $18.3 \pm 1.4$ & & & \\
\hline 77 [47] & & & & $8.3 \pm 2.3$ & $10.7 \pm 2.3$ & $4.8 \pm 2.3$ & & & \\
\hline 146 [47] & & & & $24.5 \pm 1$ & $18.6 \pm 1$ & $20.6 \pm 1$ & & & \\
\hline $\begin{array}{l}\text { doxorubicin } \\
\mathrm{HCl} \text { [47] }\end{array}$ & & & & $22.4 \pm 1.7$ & $3.4 \pm 5.1$ & $1.7 \pm 1.7$ & & & \\
\hline 58 [45] & & & & & & & 4.77 & 6.44 & 5.61 \\
\hline 87 [45] & & & & & & & 1.27 & 4.72 & 2.88 \\
\hline 97 [45] & & & & & & & 5.09 & 1.13 & 3.39 \\
\hline \multirow[t]{2}{*}{$\begin{array}{c}\text { paclitaxel } \\
\text { [45] }\end{array}$} & & & & & & & 5.98 & 3.75 & 1.88 \\
\hline & $\begin{array}{c}\text { Fibro- } \\
\text { sarcoma } \\
\text { HT-1080 }\end{array}$ & $\begin{array}{c}\text { Murine } \\
\text { colon } \\
\text { carcinoma } \\
\text { 26-L5 }\end{array}$ & $\begin{array}{l}\text { MelanomaB16- } \\
\text { BL6 }\end{array}$ & & & & & & \\
\hline 17 [26] & & 5.3 & 4.2 & & & & & & \\
\hline $50[26]$ & 27.7 & & & & & & & & \\
\hline 51 [26] & 21.6 & & & & & & & & \\
\hline 53 [26] & 3.8 & 30.2 & 20.9 & & & & & & \\
\hline 72 [26] & 11.1 & & 28.4 & & & & & & \\
\hline 111 [26] & 0.6 & 22.1 & 11.9 & & & & & & \\
\hline $112[26]$ & 0.2 & 26.6 & 9.7 & & & & & & \\
\hline 113 [26] & 0.3 & 27.7 & 11.8 & & & & & & \\
\hline $140[26]$ & 21.8 & & & & & & & & \\
\hline $\begin{array}{c}\text { 5-fluoro } \\
\text { uracil [26] }\end{array}$ & 1.5 & 0.5 & 0.6 & & & & & & \\
\hline $\begin{array}{l}\text { doxorubicin } \\
\mathrm{HCl}[26]\end{array}$ & 0.2 & 0.1 & 0.2 & $\begin{array}{l}\text { Lympho } \\
\text { cytic } \\
\text { leukemia } \\
\text { P388 }\end{array}$ & $\begin{array}{l}\text { Pancreas } \\
\text { Carci noma } \\
\text { BXPC-3 }\end{array}$ & & $\begin{array}{c}\text { CNS } \\
\text { glioblas- } \\
\text { toma } \\
\text { SF268 }\end{array}$ & $\begin{array}{c}\text { Lung } \\
\text { NCI-H460 }\end{array}$ & $\begin{array}{c}\text { Colon } \\
\text { carcinoma } \\
\text { KM20L2 }\end{array}$ \\
\hline $25[57]$ & & & & 2.1 & 2.5 & 2.8 & 2.5 & 2.5 & 2.4 \\
\hline
\end{tabular}


Table 12. Cont.

\begin{tabular}{|c|c|c|c|c|c|c|c|c|c|}
\hline $\begin{array}{l}\text { Drug } \\
\text { (Ref.) }\end{array}$ & & & & & $\begin{array}{l}\text { Cell Line } \\
\mathrm{IC}_{50}(\mu \mathrm{M})\end{array}$ & & & & \\
\hline $57[57]$ & & & & 1.8 & 1.3 & 1.3 & 1.5 & 0.5 & 0.5 \\
\hline $84[57]$ & & & & 2.0 & 1.1 & 0.7 & 0.8 & 0.3 & 0.3 \\
\hline $88[57]$ & & & & 1.7 & 1.3 & 1.8 & 1.4 & 1.8 & 1.8 \\
\hline \multirow[t]{2}{*}{$91[57]$} & & & & 3.0 & 2.0 & 1.6 & 1.5 & 1.4 & 0.6 \\
\hline & $\begin{array}{l}\text { Prostate } \\
\text { carcinoma } \\
\text { DU-145 }\end{array}$ & $\begin{array}{l}\text { Lung carci- } \\
\text { nomaA549 }\end{array}$ & $\begin{array}{c}\text { T-cell } \\
\text { leukemia } \\
\text { Jurkat }\end{array}$ & $\begin{array}{c}\text { Ovarian } \\
\text { cancer } \\
\text { Skov-3 }\end{array}$ & $\begin{array}{l}\text { Epithelial } \\
\text { colorectal } \\
\text { adenocarci- } \\
\text { noma } \\
\text { CaCo-2 }\end{array}$ & $\begin{array}{l}\text { Colon } \\
\text { cancer } \\
\text { SW480 }\end{array}$ & $\begin{array}{c}\text { Mouse } \\
\text { mam mary } \\
\text { cancer } \\
\text { EMT6 }\end{array}$ & & \\
\hline $25[57]$ & 2. 2 & & & & & & & & \\
\hline $57[57]$ & 1.1 & & & & & & & & \\
\hline $84[57]$ & 0.5 & & & & & & & & \\
\hline $88[57]$ & 1.8 & & & & & & & & \\
\hline $91[57]$ & 1.3 & & & & & & & & \\
\hline $6[50]$ & & $24.51 \pm 0.17$ & & & & & & & \\
\hline $25[50]$ & & $2.91 \pm 0.75$ & $2.85 \pm 0.16$ & $7.87 \pm 0.12$ & $3.47 \pm 0.44$ & & & & \\
\hline $26[50]$ & & & $30.07 \pm 2.49$ & & $18.98 \pm 1.16$ & & & & \\
\hline $88[50]$ & & $0.48 \pm 0.17$ & $1.96 \pm 0.44$ & $2.19 \pm 0.99$ & $2.97 \pm 0.24$ & & & & \\
\hline $161[50]$ & & $4.94 \pm 0.27$ & $4.53 \pm 0.31$ & $6.6 \pm 0.4$ & $15.2 \pm 0.3$ & & & & \\
\hline $\begin{array}{c}\text { doxorubicin } \\
{[49,50]}\end{array}$ & & $2,1 \pm 1.5$ & $0.1 \pm 0.07$ & $1.5 \pm 0.15$ & $4.3 \pm 1.9$ & 1.47 & 9.21 & & \\
\hline 158 [49] & & & & & & 14.3 & 8.6 & & \\
\hline
\end{tabular}

\section{Conclusions}

We believe that this review clearly demonstrates that Dracaena and Sansevieria species are rich sources of steroidal saponins, and have intriguing structures and interesting biological properties, including high cell antiproliferative/cytotoxic and anti-inflammatory activities. Less than $10 \%$ of the total number of known species have been subjected to scientific studies so far and more than $75 \%$ of isolated saponins were new. These data indicate that the study of Dracaena and Sansevieria saponins is still a promising field of research, highlighting the importance of extending phytochemical investigations to more species, especially in search of novel bioactive constituents. Classical methods, such as Soxhlet extraction and maceration have been used so far for the extraction of Dracaena and Sansevieria saponins. One expects that less time-consuming and greener methods, such as ultra-sound assisted extraction, microwave-assisted extraction, supercritical fluid extraction and accelerated solvent extraction that usually increase the overall efficiency of the extraction procedures, will be used more frequently in future studies. Moreover, modern dereplication methods of analysis, such as LC-MS and other hyphenated techniques [75], may allow a straightforward way to propose the tentative identification of compounds [76].

Several bioactive saponins from Dracaena and Sansevieria have the potential to become lead compounds for the development of anticancer therapeutic agents. Further studies are required to understand the relevance and selectivity of the cytotoxic activity, and to define the mechanisms of action. It is also important to access potential risks and side effects. Moreover, most of the reports were in vitro studies; therefore, further animal experiments are essential to evaluate saponin activities.

Author Contributions: Conceptualization-writing, Z.M.T.; writing-original draft preparation, S.M.O., T.M.N. and H.T.A.; review supervision-editing, G.V. and F.H.S.H.; literature retrieval, C.A. All authors have read and agreed to the published version of the manuscript. 
Funding: This research received no external funding.

Institutional Review Board Statement: Not applicable.

Informed Consent Statement: Not applicable.

Data Availability Statement: Not applicable.

Conflicts of Interest: The authors declare no conflict of interest.

Sample Availability: Samples of the compounds are not available from the authors.

\section{References}

1. Chase, M.W.; Christenhusz, M.J.M.; Fay, M.F.; Byng, J.W.; Judd, W.S.; Soltis, D.E.; Mabberley, D.J.; Sennikov, A.N.; Soltis, P.S.; Stevens, P.F.; et al. An update of the angiosperm phylogeny group classification for the orders and families of flowering plants: APG IV. Bot. J. Linn. Soc. 2016, 181, 1-20. [CrossRef]

2. Lu, P.-L.; Morden, C.W. Phylogenetic relationships among dracaenoid genera (Asparagaceae: Nolinoideae) inferred from chloroplast DNAloci. Syst. Bot. 2014, 39, 90-104. [CrossRef]

3. Takawira-Nyenya, R.; Mucina, L.; Cardinal-Mcteague, W.M.; Thiele, K.R. Sansevieria (Asparagaceae, nolinoideae) is a herbaceous clade within Dracaena: Inference from non-coding plastid and nuclear DNA sequence data. Phytotaxa 2018, 376, 254-276. [CrossRef]

4. Maděra, P.; Forrest, A.; Hanáček, P.; Vahalík, P.; Gebauer, R.; Plichta, R.; Jupa, R.; Van Rensburg, J.J.; Morris, M.; Nadezhdina, N.; et al. What we know and what we do not know about dragon trees? Forests 2020, 11, 236. [CrossRef]

5. WFO. An Online Flora of All Known Plants. Published on the Internet. 2020. Available online: http://www.worldfloraonline.org (accessed on 26 April 2020).

6. Tandu, K.R. Dracaena nitens: A new source of diosgenin. Planta Med. 1988, 54, 85. [CrossRef] [PubMed]

7. Ichikawa, K.; Kitaoka, M.; Taki, M.; Takaishi, S.; Boriboon, M.; Akiyama, T. Retrodihydrochalcones and homoisoflavones isolated from Thai medicinal plant Dracaena loureiri and their estrogen agonist activity. Planta Med. 1997, 63, 540-543. [CrossRef] [PubMed]

8. Sun, J.; Liu, J.-N.; Fan, B.; Chen, X.-N.; Pang, D.-R.; Zheng, J.; Zhang, Q.; Zhao, Y.-F.; Xiao, W.; Tu, P.-F.; et al. Phenolic constituents, pharmacological activities, quality control, and metabolism od Dracaena species: A review. J. Ethnopharmacol. 2019, 244, 439-444. [CrossRef] [PubMed]

9. Gupta, D.; Bleakley, B.; Gupta, R.K. Dragon's blood: Botany, chemistry and therapeutic uses. J. Ethnopharmacol. 2008, 115, 361-380. [CrossRef] [PubMed]

10. Ding, X.; Zhu, J.; Wang, H.; Chen, H.; Mei, W. Dragon's blood from Dracaena cambodiana in China: Applied history and induction techniques toward formation mechanism. Forests 2020, 11, 372. [CrossRef]

11. Fan, J.-Y.; Yi, T.; Sze-To, C.-M.; Zhu, L.; Peng, W.-L.; Zhang, Y.-Z.; Zhao, Z.-Z.; Chen, H.-B. A systematic review of the botanical, phytochemical and pharmacological profile of Dracaena cochinchinensis, a plant source of the ethnomedicine "dragon's blood". Molecules 2014, 19, 10650-10669. [CrossRef]

12. Machala, M.; Kubínová, R.; Hořavová, P.; Suchý, V. Chemoprotective potentials of homoisoflavonoids and chalcones of Dracaena cinnabari: Modulations of drug-metabolizing enzymes and antioxidant activity. Phytother. Res. 2001, 15, 114-118. [CrossRef]

13. Silva, B.M.; Santos, R.P.; Mendes, L.S.; de Pinho, P.G.; Valentão, P.; Andrade, P.B.; Pereira, J.A.; Carvalho, M. Dracaena draco L. fruit: Phytochemical and antioxidant activity assessment. Food. Res. Int. 2011, 44, 2182-2189. [CrossRef]

14. Mbugua, P.K.; Moore, D.M. Taxonomic studies of the genus Sansevieria (Dracaenaceae). In The Biodiversity of African Plants; Van der Maesen, L.J.G., Van der Burgt, M., Van Medenbach de Rooy, J.M., Eds.; Springer: Dordrecht, The Netherlands, 1996; pp. 489-492.

15. Takawira, R.; Nordal, I. The genus of Sansevieria (family Dracaenaceae) in Zimbabwe. Acta Hortic. 2002, 572, 189-198. [CrossRef]

16. Andhare, R.N.; Raut, M.K.; Naik, S.R. Evaluation of antiallergic and anti-anaphylactic activity of ethanolic extract of Sanseveiria trifasciata leaves (EEST) in rodents. J. Ethnopharmacol. 2012, 142, 627-633. [CrossRef] [PubMed]

17. Bero, J.; Ganfon, H.; Jonville, M.-C.; Frédérich, M.; Gbaguidi, F.; DeMol, P.; Moudachirou, M.; Quetin-Leclercq, J. In vitro antiplasmodial activity of plants used in Benin in traditional medicine to treat malaria. J. Ethnopharmacol. 2009, 122, 439-444. [CrossRef] [PubMed]

18. Kpodar, M.S.; Karou, S.D.; Katawa, G.; Anani, K.; Gbekley, H.E.; Adjrah, Y.; Tchacondo, T.; Batawila, K.; Simpore, J. An ethnobotanical study of plants used to treat liver diseases in the maritime region of Togo. J. Ethnopharmacol. 2016, 181, $263-273$. [CrossRef]

19. Giovannini, P.; Howes, M.-J.R. Medicinal plants used to treat snakebite in Central America: Review and assessment of scientific evidence. J. Ethnopharmacol. 2017, 199, 240-256. [CrossRef]

20. Lekawatana, S.; Suwannamek, B. Ornamental plants in Thailand. Acta Hortic. 2017, 1167, 11-16. [CrossRef]

21. Saxena, P.; Ghosh, C. Ornamental plants as sinks and bioindicators. Environ. Technol. 2013, 34, 3059-3067. [CrossRef]

22. Thu, Z.M.; Myo, K.K.; Aung, H.T.; Armijos, C.; Vidari, G. Flavonoids and stilbenoids of the genera Dracaena and Sansevieria: Structures and bioactivities. Molecules 2020, 25, 2608. [CrossRef] [PubMed] 
23. Simmons-Boyce, J.L.; Tinto, W.F. Steroidal saponins and sapogenins from the agavaceae family. Nat. Prod. Commun. 2007, 2, 99-114. [CrossRef]

24. Sahu, N.P.; Banerjee, S.; Mondal, N.B.; Mandal, D. Steroidal Saponins. In Fortschritte der Chemie Organischer Naturstoffe/Progress in the Chemistry of Organic Natural Products; Kinghorn, A.D., Falk, H., Kobayashi, J., Eds.; Springer: Vienna, Austria, 2008; Volume 89, pp. 45-141.

25. Sidana, J.; Singh, B.; Sharma, O.P. Saponins of Agave: Chemistry and bioactivity. Phytochemistry 2016, 130, 22-46. [CrossRef]

26. Tran, Q.L.; Tezuka, Y.; Banskota, A.H.; Tran, Q.K.; Saiki, I.; Kadota, S. New spirostanol steroids and steroidal saponins from roots and rhizomes of Dracaena angustifolia and their antiproliferative activity. J. Nat. Prod. 2001, 64, 1127-1132. [CrossRef] [PubMed]

27. Kougan, G.B.; Miyamoto, T.; Tanaka, C.; Paululat, T.; Mirjolet, J.-F.; Duchamp, O.; Sondengam, B.L.; Lacaille-Dubois, M.-A. Steroidal saponins from two species of Dracaena. J. Nat. Prod. 2010, 73, 1266-1270. [CrossRef]

28. González, A.G.; Hernández, J.C.; León, F.; Padrón, J.I.; Estévez, F.; Quintana, J.; Bermejo, J. Steroidal saponins from the bark of Dracaena draco and their cytotoxic activities. J. Nat. Prod. 2003, 66, 793-798. [CrossRef]

29. Hernández, J.C.; León, F.; Estévez, F.; Quintana, J.; Bermejo, J. A homo-isoflavonoid and a cytotoxic saponin from Dracaena draco. Chem. Biodivers. 2006, 3, 62-68. [CrossRef] [PubMed]

30. Moharram, F.A.; EI-Shenawy, S.M. Antinociceptive and anti-inflammatory steroidal saponins from Dracaena ombet. Planta Med. 2007, 73, 1101-1106. [CrossRef] [PubMed]

31. Yokosuka, A.; Mimaki, Y.; Sashida, Y. Steroidal saponins from Dracaena surculosa. J. Nat. Prod. 2000, 63, 1239-1243. [CrossRef] [PubMed]

32. Tang, L.; Wang, Z.; Wu, H.; Yokosuka, A.; Mimaki, Y. Steroidal glycosides from the underground parts of Dracaena thalioides and their cytotoxic activity. Phytochemistry 2014, 107, 102-110. [CrossRef] [PubMed]

33. Teponno, R.B.; Tanaka, C.; Jie, B.; Tapondjou, L.A.; Miyamoto, T. Trifasciatosides A-J, steroidal saponins from Sansevieria trifasciata. Chem. Pharm. Bull. 2016, 64, 1347-1355. [CrossRef]

34. Agrawal, P.K.; Jain, D.C.; Gupta, R.K.; Thakur, R.S. Carbon-13 NMR spectroscopy of steroidal sapogenins and steroidal saponins. Phytochemistry 1985, 24, 2479-2496. [CrossRef]

35. Agrawal, P.K.; Jain, D.C.; Pathak, A.K. NMR spectroscopy of steroidal sapogenins and steroidal saponins: An update. Magn. Reson. Chem. 1995, 33, 923-953. [CrossRef]

36. Agrawal, P.K.; Bunsawansong, P.; Morris, G.A. Dependence of the ${ }^{1} \mathrm{H}$ NMR chemical shifts of ring F resonances on the orientation of the 27-methyl group of spirostane-type steroidal sapogenins. Phytochemistry 1998, 47, 2479-2496. [CrossRef]

37. Agrawal, P.K. Spectral assignments and reference data: $25 R / 25 S$ stereochemistry of spirostane-type steroidal sapogenins and steroidal saponins via chemical shift of geminal protons of ring-F. Magn. Reson. Chem. 2003, 41, 965-968. [CrossRef]

38. Agrawal, P.K. Dependence of ${ }^{1} \mathrm{H}$ NMR chemical shifts of geminal protons of glycosyloxy methylene $\left(\mathrm{H}_{2}-26\right)$ on the orientation of the 27-methyl group of furostane-type steroidal saponins. Magn. Reson. Chem. 2004, 42, 990-993. [CrossRef] [PubMed]

39. Agrawal, P.K. Assigning stereodiversity of the 27-Me group of furostane-type steroidal saponins via NMR chemical shifts. Steroids 2005, 70, 715-724. [CrossRef]

40. Agrawal, P.K. NMR Spectroscopy in the structural elucidation of oligosaccharides and glycosides. Phytochemistry 1992, 31,3307-3330. [CrossRef]

41. Mandal, D.; Banerjee, S.; Mondal, N.B.; Chakravarty, A.K.; Sahu, N.P. Steroidal saponins from the fruits of Asparagus racemosus. Phytochemistry 2006, 67, 1316-1321. [CrossRef] [PubMed]

42. Claridge, T.D.W. High-Resolution NMR Techniques in Organic Chemistry, 3rd ed.; Elsevier Science: Oxford, UK, 2016.

43. Kessler, H.; Gehrke, M.; Griesinger, C. Two-dimensional NMR spectroscopy: Background and overview of the experiments. Angew. Chem. Int. Ed. Engl. 1988, 27, 490-536. [CrossRef]

44. Bross-Walch, N.; Kühn, T.; Moskau, D.; Zerbe, O. Strategies and tools for structure determination of natural products using modern methods of NMR spectroscopy. Chem. Biodivers. 2005, 2, 147-177. [CrossRef] [PubMed]

45. Shen, H.-Y.; Zuo, W.-J.; Wang, H.; Zhao, Y.-X.; Guo, Z.-K.; Luo, Y.; Li, X.-N.; Dai, H.-F.; Mei, W.-L. Steroidal saponins from dragon's blood of Dracaena cambodiana. Fitoterapia 2014, 94, 94-101. [CrossRef]

46. Rezgui, A.; Mitaine-Offer, A.-C.; Miyamoto, T.; Tanaka, C.; Lacaille-Dubois, M.-A. Spirostane-type saponins from Dracaena fragrans « Yellow Coast ». Nat. Prod. Commun. 2015, 10, 37-38. [CrossRef]

47. Raslan, M.A.; Melek, F.R.; Said, A.A.; Elshamy, A.I.; Umeyama, A.; Mounier, M.M. New cytotoxic dihydrochalcone and steroidal saponins from the aerial parts of Sansevieria cylindrica Bojer ex Hook. Phytochem. Lett. 2017, 22, 39-43. [CrossRef]

48. Xu, M.; Zhang, Y.-J.; Li, X.-C.; Jacob, M.R.; Yang, C.-R. Steroidal saponins from fresh stems of Dracaena angustifolia. J. Nat. Prod. 2010, 73, 1524-1528. [CrossRef]

49. Rezgui, A.; Mitaine-Offer, A.-C.; Pertuit, D.; Miyamoto, T.; Tanaka, C.; Delemasure, S.; Dutartre, P.; Lacaille-Dubois, M.-A. Steroidal saponins from Dracaena marginata. Nat. Prod. Commun. 2013, 8, 157-160. [CrossRef] [PubMed]

50. Teponno, R.B.; Dzoyem, J.P.; Nono, R.N.; Kauhl, U.; Sandjo, L.P.; Tapondjou, L.A.; Bakowsky, U.; Opatz, T. Cytotoxicity of secondary metabolites from Dracaena viridiflora Engl \& Krause and their semisynthetic analogues. Rec. Nat. Prod. 2017, 11, 421-430.

51. Tapondjou, L.A.; Ponou, K.B.; Teponno, R.B.; Mbiantcha, M.; Djoukeng, J.D.; Nguelefack, T.B.; Watcho, P.; Cadenas, A.G.; Park, H.-J. In vivo anti-inflammatory effect of a new steroidal saponin, mannioside A, and its derivatives isolated from Dracaena mannii. Arch. Pharm. Res. 2008, 31, 653. [CrossRef] 
52. Yokosuka, A.; Mimaki, Y.; Sashida, Y. Four new 3,5-cyclosteroidal saponins from Dracaena surculosa. Chem. Pharm. Bull. 2002, 50, 992-995. [CrossRef] [PubMed]

53. Mimaki, Y.; Kuroda, M.; Ide, A.; Kameyama, A.; Yokosuka, A.; Sashida, Y. Steroidal saponins from the aerial parts of Dracaena draco and their cytostatic activity on HL-60 cells. Phytochemistry 1999, 50, 805-813. [CrossRef]

54. Tchegnitegni, B.T.; Teponno, R.B.; Tanaka, C.; Gabriel, A.F.; Tapondjou, L.A.; Miyamoto, T. Sappanin-type homoisoflavonoids from Sansevieria trifasciata Prain. Phytochem. Lett. 2015, 12, 262-266. [CrossRef]

55. Hernández, J.C.; León, F.; Quintana, J.; Estévez, F.; Bermejo, J. Icogenin, a new cytotoxic steroidal saponin isolated from Dracaena draco. Bioorg. Med. Chem. 2004, 12, 4423-4429. [CrossRef] [PubMed]

56. Xu, M.; Yang, C.-R.; Zhang, Y.-J. New $\mathrm{C}_{27}$ steroidal bisdesmosides from the fresh stems of Dracaena cambodiana. Helv. Chim. Acta 2010, 93, 302-308. [CrossRef]

57. Pettit, G.R.; Zhang, Q.; Pinilla, V.; Hoffmann, H.; Knight, J.C.; Doubek, D.L.; Chapuis, J.-C.; Pettit, R.K.; Schmidt, J.M. Antineoplastic agents. 534. Isolation and structure of Sansevistatins 1 and 2 from the African Sansevieria ehrenbergii,1. J. Nat. Prod. 2005, 68, 729-733. [CrossRef]

58. Huang, H.-C.; Lin, M.-K.; Hwang, S.-Y.; Hwang, T.-L.; Kuo, Y.-H.; Chang, C.-I.; Ou, C.-Y.; Kuo, Y.-H. Two anti-inflammatory steroidal saponins from Dracaena angustifolia Roxb. Molecules 2013, 18, 8752-8763. [CrossRef]

59. Mimaki, Y.; Kuroda, M.; Takaashi, Y.; Sashida, Y. Steroidal saponins from the stems of Dracaena concinna. Phytochemistry 1998, 47, 1351-1356. [CrossRef]

60. Said, A.; Aboutabl, E.A.; Melek, F.R.; Abdel Jaleel, G.A.R.; Raslan, M. Steroidal saponins and homoisoflavanone from the aerial parts of Sansevieria cylindrica Bojer ex Hook. Phytochem. Lett. 2015, 12, 113-118. [CrossRef]

61. Zheng, Q.-A.; Zhang, Y.-J.; Li, H.-Z.; Yang, C.-R. Steroidal saponins from fresh stem of Dracaena cochinchinensis. Steroids 2004, 69, 111-119. [CrossRef]

62. Luo, Y.; Shen, H.-Y.; Zuo, W.-J.; Wang, H.; Mei, W.-L.; Dai, H.-F. A new steroidal saponin from dragon's blood of Dracaena cambodiana. J. Asian Nat. Prod. Res. 2014, 17, 409-414. [CrossRef]

63. Mimaki, Y.; Inoue, T.; Kuroda, M.; Sashida, Y. Steroidal saponins from Sansevieria trifasciata. Phytochemistry 1996, 43, 1325-1331. [CrossRef]

64. Tchegnitegni, B.T.; Teponno, R.B.; Jenett-Siems, K.; Melzig, M.F.; Miyamoto, T.; Tapondjou, L.A. A dihydrochalcone derivative and further steroidal saponins from Sansevieria trifasciata Prain. Z. Nat. C 2017, 72, 477-482. [CrossRef]

65. Okunji, C.O.; Okeke, C.N.; Gugnani, H.C.; Iwu, M.M. An antifungal spirostanol saponin from fruit pulp of Dracaena mannii. Int. J. Crude Drug Res. 1990, 28, 193-199. [CrossRef]

66. Okunji, C.O.; Iwu, M.M.; Hostettmann, K. Molluscicidal saponins from the fruit pulp of Dracaena mannii. Int. J. Pharmacogn. 1991, 29, 66-70. [CrossRef]

67. Yokosuka, A.; Sekiguchi, A.; Mimaki, Y. Chemical constituents of the leaves of Dracaena thalioides. Nat. Prod. Commun. 2013, 8, 315-318. [CrossRef]

68. Reddy, K.S.; Shekhani, M.S.; Berry, D.E.; Lynn, D.G.; Hecht, S.M. Afromontoside. A new cytotoxic principle from Dracaena afromontana. J. Chem. Soc. Perkin Trans. I 1984, 987-992. [CrossRef]

69. da Silva Antunes, A.; da Silva, B.P.; Parente, J.P.; Valente, A.P. A new bioactive steroidal saponin from Sansevieria cylindrica. Phytother. Res. 2003, 17, 179-182. [CrossRef] [PubMed]

70. Zheng, Q.A.; Yang, C.R. Dracaenoside A and B, new C-22 steroidal lactone glycosides from the stem of Dracaena cochinchinensis. Chin. Chem. Lett. 2003, 14, 1261-1264.

71. Mimaki, Y.; Inoue, T.; Kuroda, M.; Sashida, Y. Pregnane glycosides from Sansevieria trifasciata. Phytochemistry 1997, 44, 107-111. [CrossRef]

72. Zheng, Q.-A.; Yang, C.-R. Pregnane glycosides from Dracaena cochinchinensis. J. Asian Nat. Prod. Res. 2003, 5, 291-296. [CrossRef]

73. Podolak, I.; Galanty, A.; Sobolewska, D. Saponins as cytotoxic agents: A review. Phytochem. Rev. 2010, 9, 425-474. [CrossRef]

74. Min, H.-Y.; Pei, H.; Hyun, S.Y.; Boo, H.-J.; Jang, H.-J.; Cho, J.; Kim, J.H.; Son, J.; Lee, H.-Y. Potent anticancer effect of the natural steroidal saponin gracillin is produced by inhibiting glycolysis and oxidative phosphorylation-mediated bioenergetics. Cancers 2020, 12, 913. [CrossRef]

75. Crotti, A.E.M.; Carollo, C.A.; Gobbo-Neto, L.; Santos, M.D.; Gates, P.J.; Lopes, N.P. LC-hyphenated techniques: Uses in the structural elucidation of low and high molecular weight compounds. In Modern Biotechnology in Medicinal Chemistry and Industry; Taft, C.A., Ed.; Research Signpost: Kerala, India, 2006; Volume 61, pp. 99-141.

76. Mendonça, S.C.; Simas, R.C.; Simas, D.L.R.; Leitão, S.G.; Leitão, G.G. Mass spectrometry as a tool for the dereplication of saponins from Ampelozizyphus amazonicus Ducke bark and wood. Phytochem. Anal. 2020, 1-21. [CrossRef] [PubMed] 